Florida International University FIU Digital Commons

6-28-1998

\title{
Yttria stabilized zirconia buffered silicon: substrates for YBCO microwave applications
}

Philip D. Brown

Florida International University

DOI: $10.25148 /$ etd.FI14051862

Follow this and additional works at: https://digitalcommons.fiu.edu/etd

Part of the Electrical and Electronics Commons

\section{Recommended Citation}

Brown, Philip D., "Yttria stabilized zirconia buffered silicon: substrates for YBCO microwave applications" (1998). FIU Electronic Theses and Dissertations. 1878.

https://digitalcommons.fiu.edu/etd/1878

This work is brought to you for free and open access by the University Graduate School at FIU Digital Commons. It has been accepted for inclusion in FIU Electronic Theses and Dissertations by an authorized administrator of FIU Digital Commons. For more information, please contact dcc@fiu.edu. 


\title{
FLORIDA INTERNATIONAL UNIVERSITY
}

\author{
Miami, Florida
}

\section{YTTRIA STABILIZED ZIRCONIA BUFFERED SILICON: SUBSTRATES FOR YBCO MICROWAVE APPLICATIONS}

\author{
A thesis submitted in partial fulfillment of the \\ requirements for the degree of \\ MASTER OF SCIENCE \\ in
}

ELECTRICAL ENGINEERING

by

Philip D. Brown 
To: Dean Gordon Hopkins

College of Engineering

This thesis, written by Philip D. Brown, and entitled Yttria Stabilized Zirconia Buffered Silicon: Substrates for YBCO Microwave Applications, having been approved in respect to style and intellectual content is referred to you for judgment.

We have read this thesis and recommend that it be approved.

Carolyne Van Vliet

Jean Andrian

Yuri Vlasov

Date of Defense: June 28, 2000

Grover Larkins, Major Professor

The thesis of Philip D. Brown is approved.

Dr. Gordon R. Hopkins, Dean College of Engineering

Dean Richard L. Campbell Division of Graduate Studies 


\section{DEDICATION}

I dedicate this thesis to my parents, for their understanding, patience, love and support. 


\section{ACKNOWLEDGMENTS}

I wish to thank my major professor Dr. Grover Larkins and the members of my committee, Dr. Yuri Vlasov, Dr. Carolyne Van-Vliet and Dr. Jean Andrian for all their patience, encouragement and support. A special thanks to Mr. Ed Maltasse for teaching me so many vital laboratory procedures. I would also like to thank my entire family who has always been very supportive.

I owe much to all the members of the FAST center team for their help and support with this project. It is not possible to thank all: to Peter Batista, Thaer Sayed, Nadia Khan, Dane Fairweather and Erika Collantes for assistance with experiments and data collection and to Elvira Chang, Dianne Dixon, and Melissa for making things happen. I am especially grateful for many helpful suggestions and invaluable advice from Tasharruf Khan and Robert Soto, whom without, this work would not have become a reality. Also to Pat, Marbeth and Nancy for their immense help.

A very special thanks to Dr. Patricia Stampe and Dr. Robin Kennedy for providing $\mathrm{x}$-ray diffraction results and ellipsometry measurements.

This work was supported by the Air Force Office of Scientific Research under Grant No. F49620-95-0519. 
ABSTRACT OF THESIS

YTTRIA STABILIZED ZIRCONIA BUFFERED SILICON: SUBSTRATES FOR YBCO

MICROWAVE APPLICATIONS

by

Philip Brown

Florida International University, 2000

Miami, Florida

Professor Grover Larkins, Major Professor

The use Yttria-Stabilized Zirconia (YSZ) as a buffer layer for $\mathrm{YBa}_{2} \mathrm{Cu}_{3} \mathrm{O}_{7-\mathrm{x}}(\mathrm{YBCO})$ thin films on (100) silicon ( $\mathrm{Si}$ ) substrates is investigated. YSZ was grown using on-axis pulsed D.C. (PDC) and R.F. magnetron sputtering from a 99.9\% pure YSZ target $\left[\left(\mathrm{Y}_{2} \mathrm{O}_{3}\right)_{0.018}\left(\mathrm{ZrO}_{2}\right)_{1.92}\right]$. Sputtering was carried out in Argon/Oxygen atmosphere at total pressures ranging from 6 to 320 mTorr containing 0.1 to $10 \%$ Oxygen. Substrate temperatures were varied from $300^{\circ} \mathrm{C}$ to $900^{\circ} \mathrm{C}$. (111), (100) and mixed (100) and (111) oriented YSZ films were produced with thickness of 50 to $200 \mathrm{~nm}$. YBCO films, $200 \mathrm{~nm}$ thick, were then grown by pulsed-laser deposition in an atmosphere of 0.5 Torr $\mathrm{O}_{2}$ at $750^{\circ} \mathrm{C}$. The critical temperature $\left(\mathrm{T}_{\mathrm{C}(\mathrm{R}=11)}\right)$ of the films produced was $75 \mathrm{~K}-81 \mathrm{~K}$. A " $\mathrm{T}$ " resonator design was patterned on the YBCO/YSZ/Si structure and tested. Results show a bandreject response centered at $3.872 \mathrm{GHz}$ with a quality factor of 20,000 . 


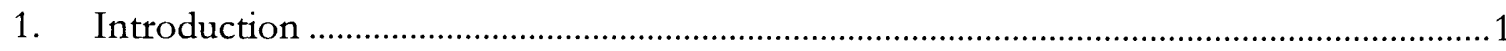

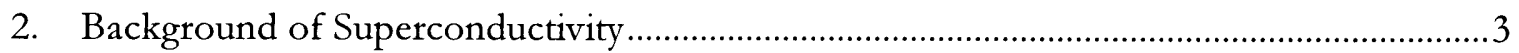

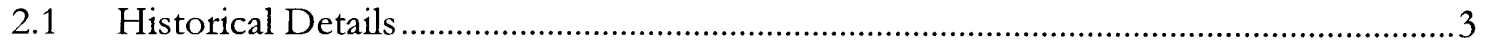

2.2 Low Temperature vs. High Temperature Superconductors ...........................................6

2.2.1 Large Scale Applications......................................................................................

2.2.2 Small Scale Applications ....................................................................................

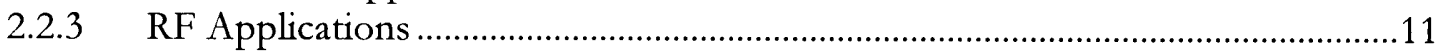

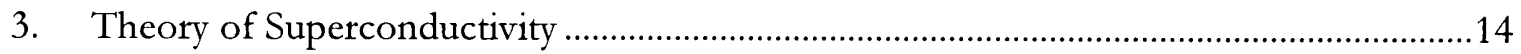

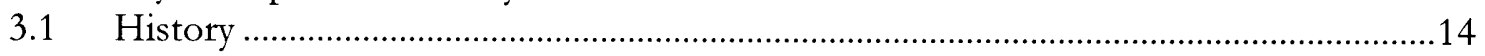

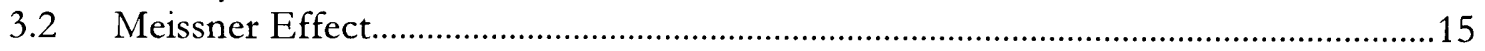

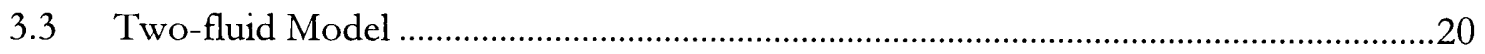

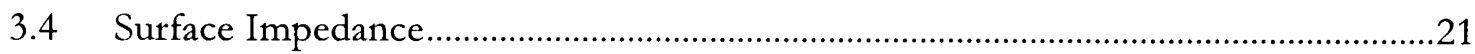

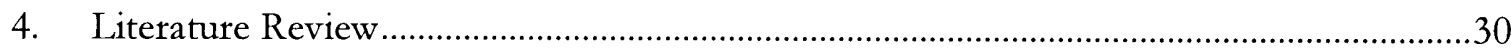

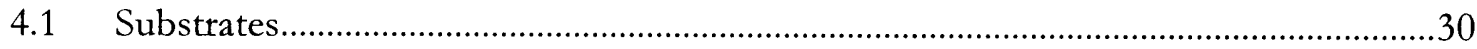

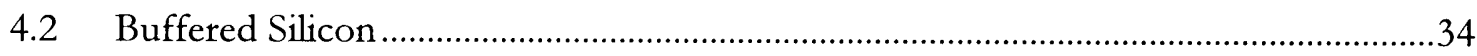

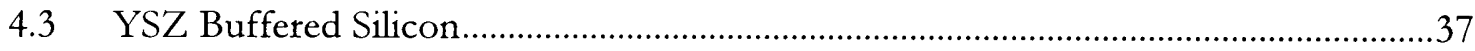

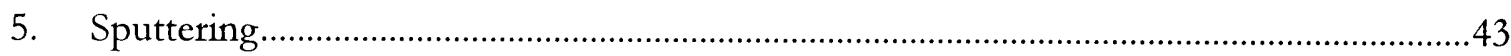

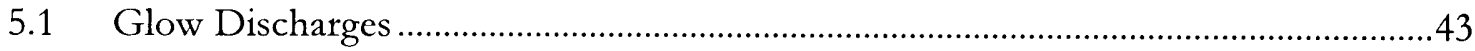

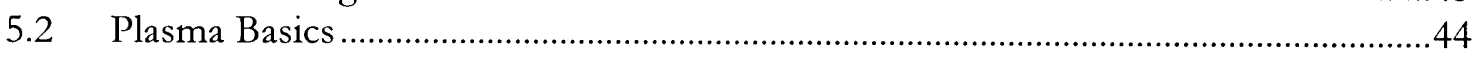

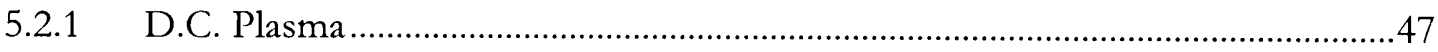

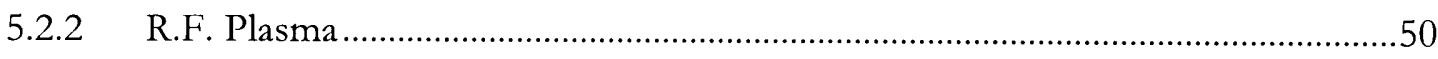

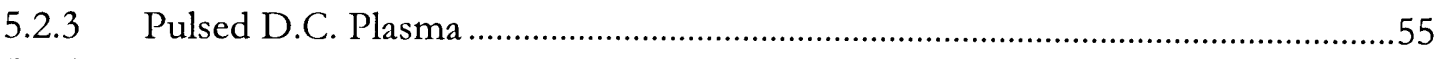

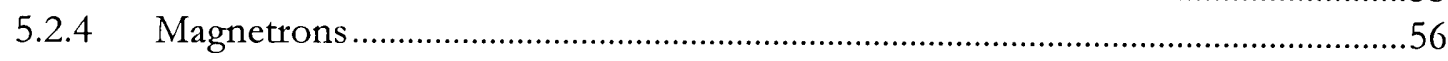

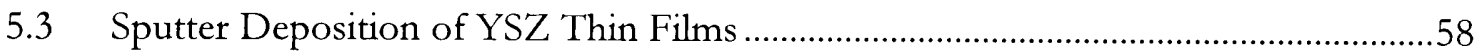

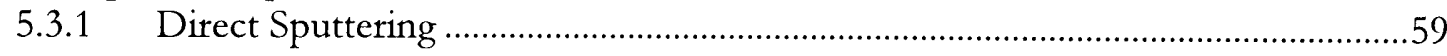

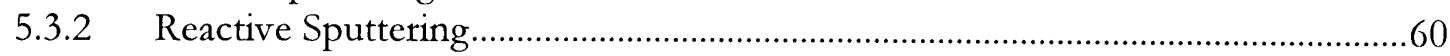

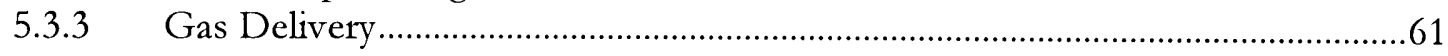

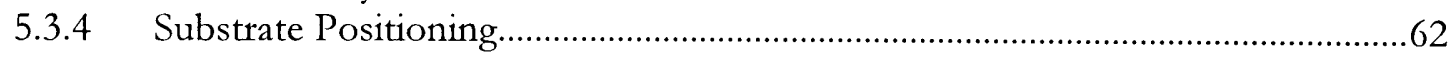

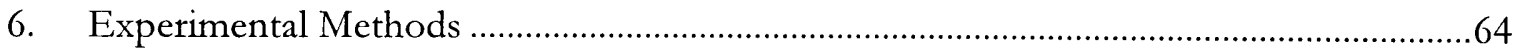

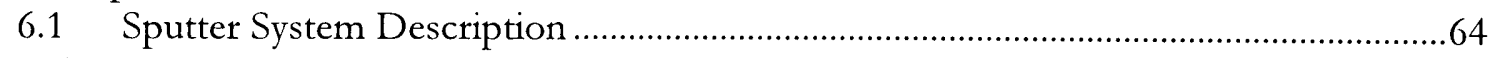

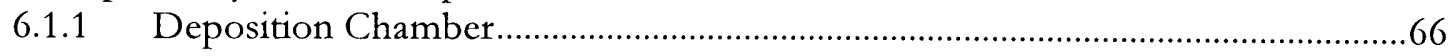

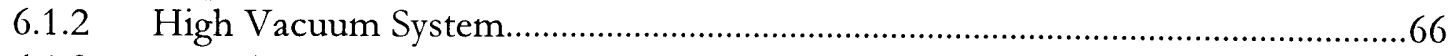

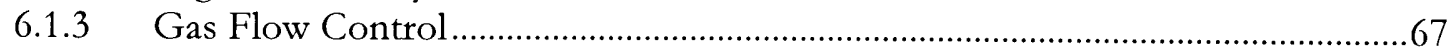

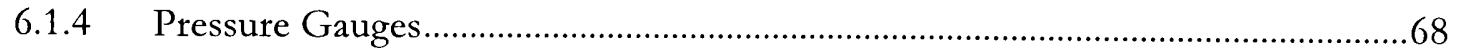




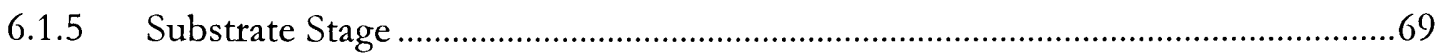

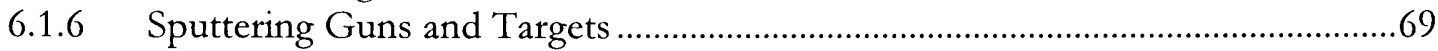

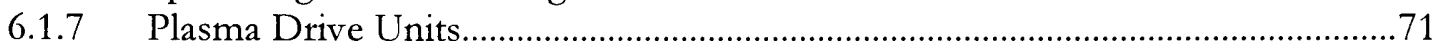

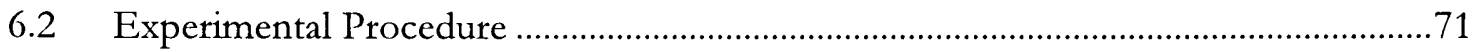

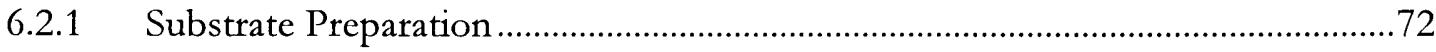

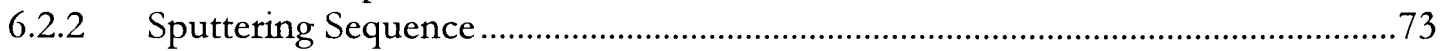

6.2.3 Deposition Parameters .......................................................................................75

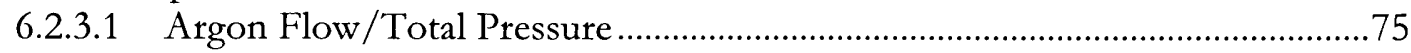

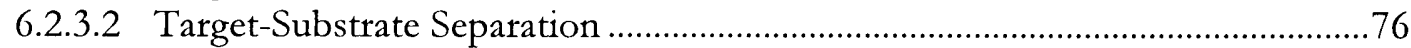

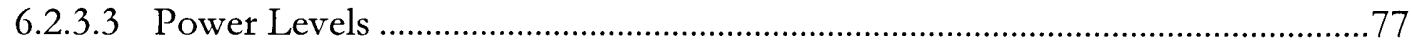

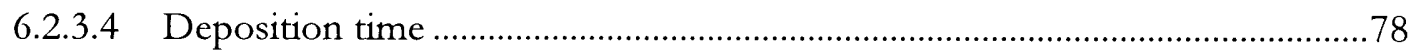

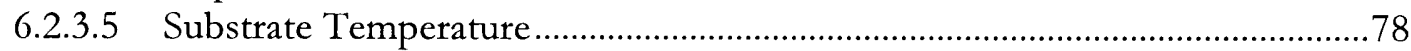

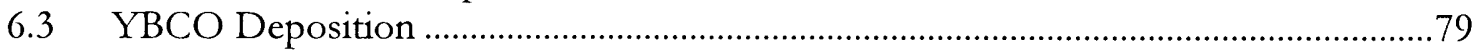

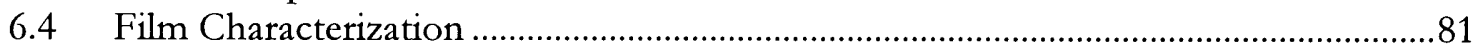

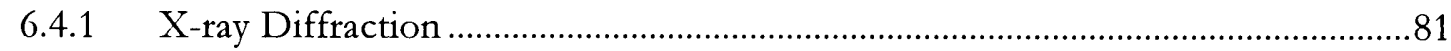

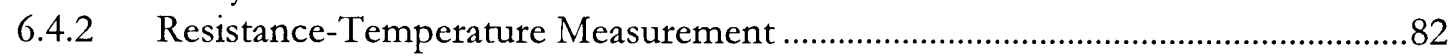

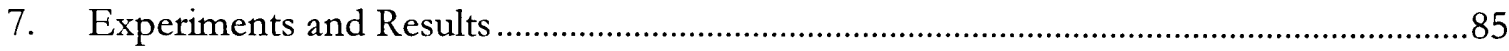

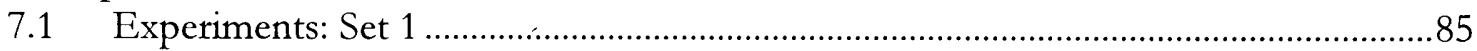

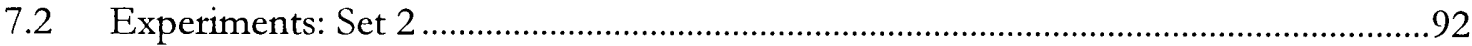

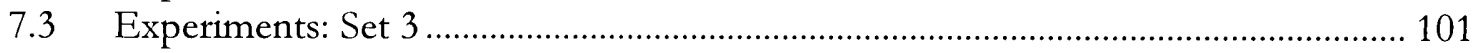

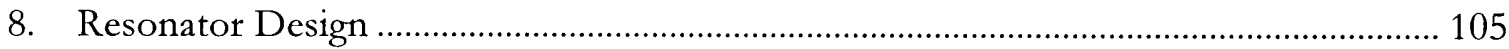

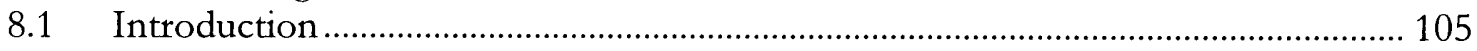

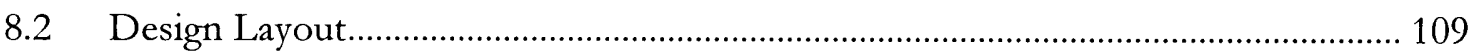

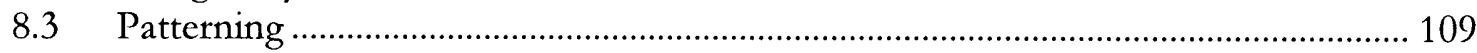

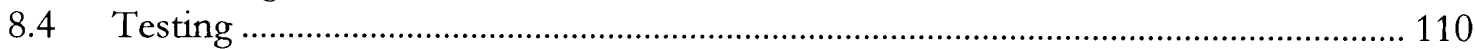

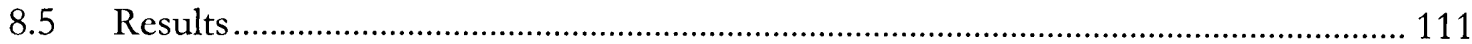

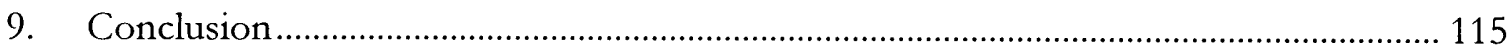

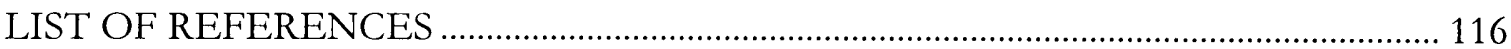




\section{LIST OF FIGURES}

FIGURE

PAGE

2.1. Resistance vs. Temperature of Mercury ..................................................................

2.2. Superconductive materials and their year of discovery ........................................6

2.1.2. Small-scale applications of superconductors.......................................................

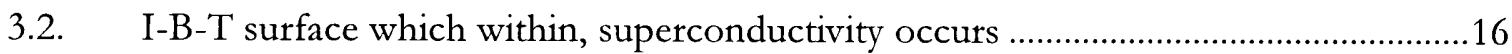

3.4. Surface resistivity of Copper and YBCO at 77K K...............................................29

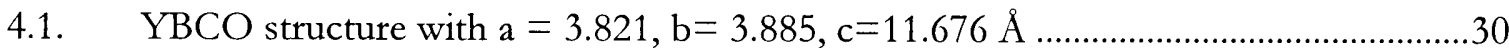

5.2 (a). Initial state before sheath formation ..................................................................46

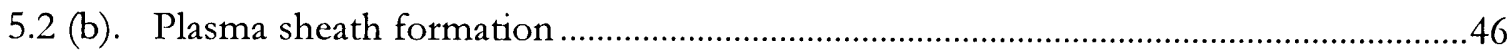

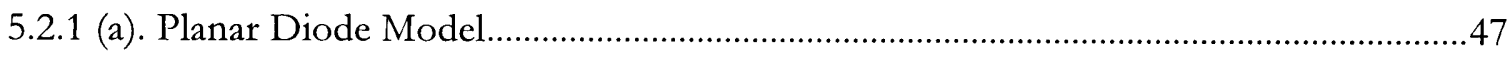

5.2.1 (b). Voltage profile for Parallel Plate Model .................................................................48

5.2 .2 (a). Low Frequency Voltage vs. Time .........................................................................5

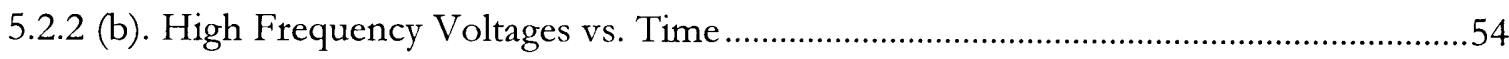

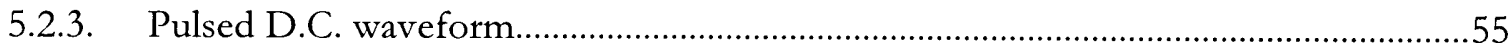

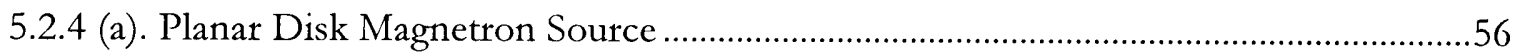

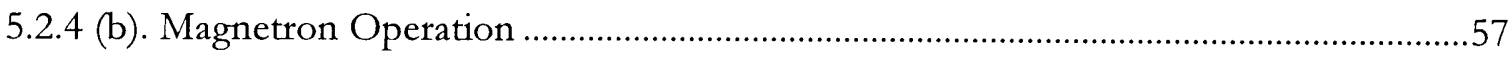

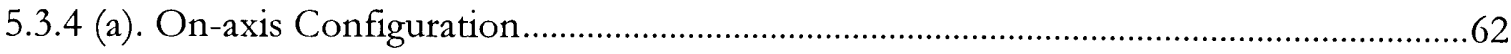

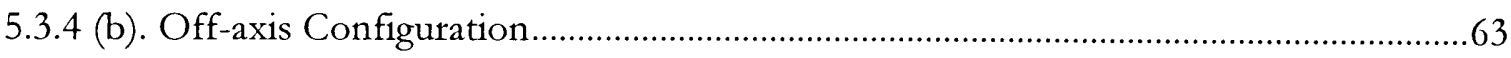

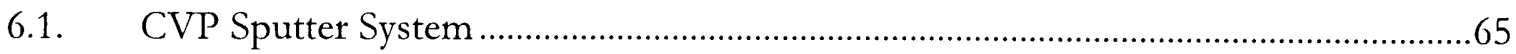

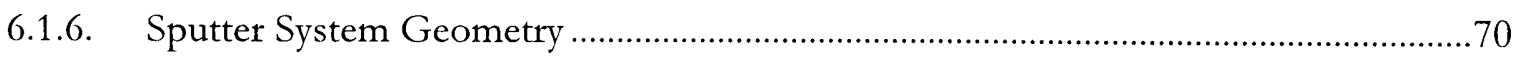

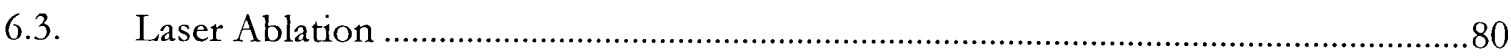

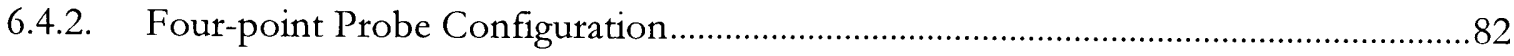


7.1 (a). X-ray spectrum of sample \#1 $\left(440^{\circ} \mathrm{C}\right)$

7.1 (b). Rocking Curve of sample \#1 .87

7.1 (c). X-ray spectrum of sample $\# 2\left(225^{\circ} \mathrm{C}\right)$ 87

7.1 (d). X-ray scan of sample \#4. .88

7.1 (e). Rocking curve for sample \#4. .89

7.1 (f). $R$ vs. T for sample \#1 .90

7.1 (g). R vs. T for sample \#2 .90

7.2 (a). X-ray scan of sample \#8. .93

7.2 (b). Phi-plot of sample \#8. .93

7.2 (c). $\mathrm{R}$ vs. T of sample \#8. 94

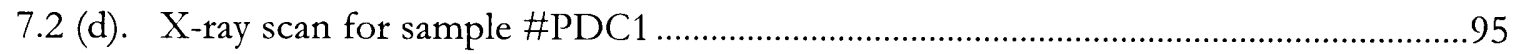

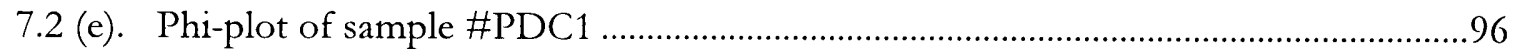

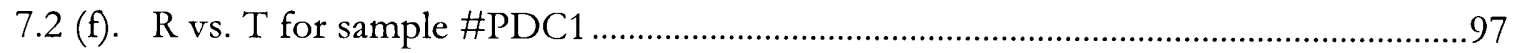

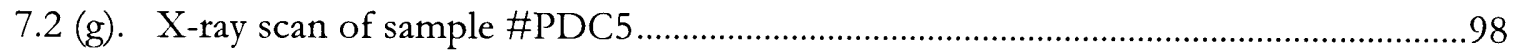

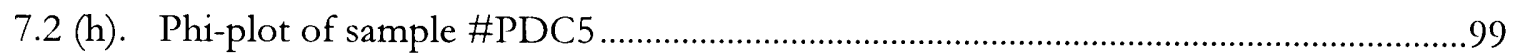

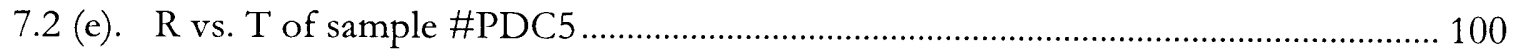

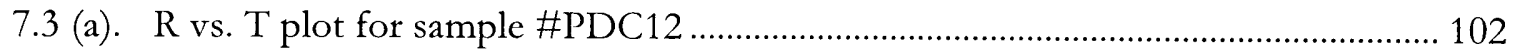

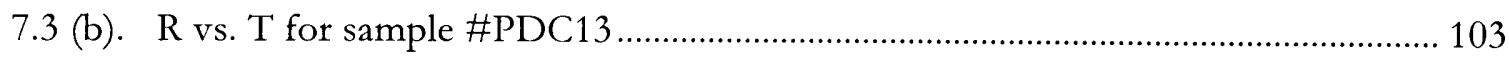

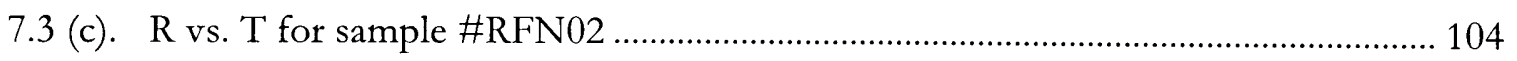

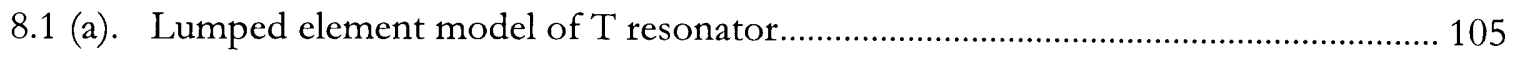

8.1 (b). Co-planar Waveguide Transmission Line .................................................................... 106

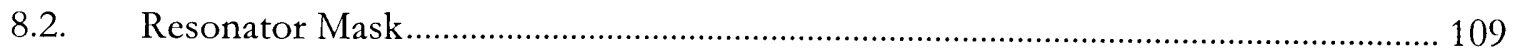

8.5(a). Experimental Resonator Response …………….................................................... 112 


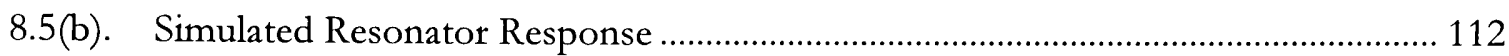

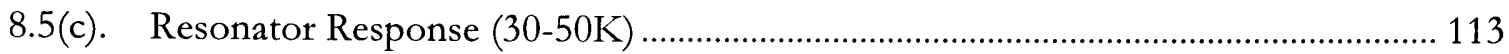

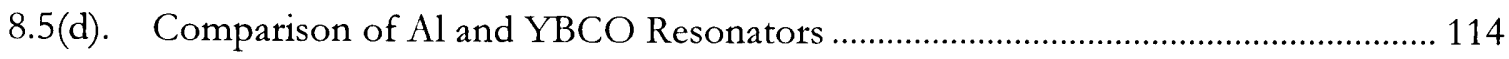


The use of high temperature superconductors in a variety of applications has been rapidly developing. In particular, communication electronics and power generation and distribution are two of the most widely researched for commercial use. Popular high temperature superconductors used are: $\mathrm{Bi}_{2} \mathrm{Sr}_{2} \mathrm{CaCu}_{2} \mathrm{O}_{8}$ (BSCCO), $\mathrm{YBa}_{2} \mathrm{Cu}_{3} \mathrm{O}_{7}(\mathrm{YBCO})$ and $\mathrm{Tl}_{2} \mathrm{Ba}_{2} \mathrm{Ca}_{2} \mathrm{Cu}_{3} \mathrm{O}_{10}$ (TBCCO). For high frequency applications YBCO is a popular choice due to its ease of preparation and high power handling capability [1].

In designing planar circuits such as microwave resonators/filters, a suitable substrate for growth of YBCO is needed. These include single substrates or substrates with a buffer layer to provide a barrier to inter-diffusion and chemical reactions. In particular silicon, with a buffer layer, is of interest for the development of hybrid superconductor-semiconductor devices [1-3].

In this thesis, silicon with a buffer layer of yttria-stabilized zirconia (YSZ) is studied as a substrate for YBCO thin films. Experiments were carried out to determine suitable parameters for depositing YSZ buffer layers on silicon. The most common methods for depositing such buffer layers include electron-beam evaporation, pulsed-laser ablation and magnetron sputtering. In this work the methods of deposition used included R.F. magnetron and pulsed D.C. magnetron sputtering. The ultimate goal was to prepare $\mathrm{YBCO} / \mathrm{YSZ} / \mathrm{Si}$ samples that could then be pattered with high frequency planar structures and their microwave performance evaluated. 
This project is comprised of three parts. The first part consists of a search for optimal deposition parameters to produce good quality YSZ films on silicon. The second part includes the evaluation of the resulting films through film characterization methods such as $\mathrm{x}$-ray diffraction. This provided a way to further fine tune the deposition parameters to optimize the growth of the YSZ film. For the YBCO, films were characterized by their resistance-temperature characteristic providing an additional method for evaluating the quality of the YSZ layer. The third part entails the patterning of a coplanar waveguide "T" resonator on to a $\mathrm{YBCO} / \mathrm{YSZ} / \mathrm{Si}$ sample and evaluating its microwave performance.

The following gives a brief overview of what each chapter covers. Chapter 2 gives some background on superconductivity and its applications. Chapter 3 goes through some basic theories of superconductivity and some electromagnetic characteristics of superconductors at high frequencies. In chapter 4 , a review of the literature on substrate materials is given, particularly YSZ buffered silicon substrates for YBCO thin films. Methods of deposition are discussed and the results compared. Chapter 5 explains the method of sputtering with details on R.F. and pulsed D.C. magnetron variety.

Chapter 6 provides detailed descriptions of the experimental techniques used, while chapter 7 enumerates the experiments carried out and the results obtained. Chapter 8 supplies details on the " $T$ " resonator and results of microwave testing are shown. Conclusions and a discussion of future work are given in chapter 9 . 


\section{BACKGROUND OF SUPERCONDUCTIVITY}

\subsection{Historical Details}

In 1911 Dutch scientist Heike Kamerlingh Onnes, discovered that mercury (Hg) lost all electrical resistance when cooled to the temperature of liquid helium [4]. It was observed that while being cooled, the mercury's resistance decreased linearly with temperature. At a temperature of $4.2 \mathrm{~K}\left(-268.8^{\circ} \mathrm{C}\right)$ above absolute zero, all resistance was lost as shown in figure 2.1. This characterized mercury as the first superconductor, with the phenomena occurring at a critical temperature $\left(\mathrm{T}_{c}\right)$ equal to $4.2 \mathrm{~K}$.

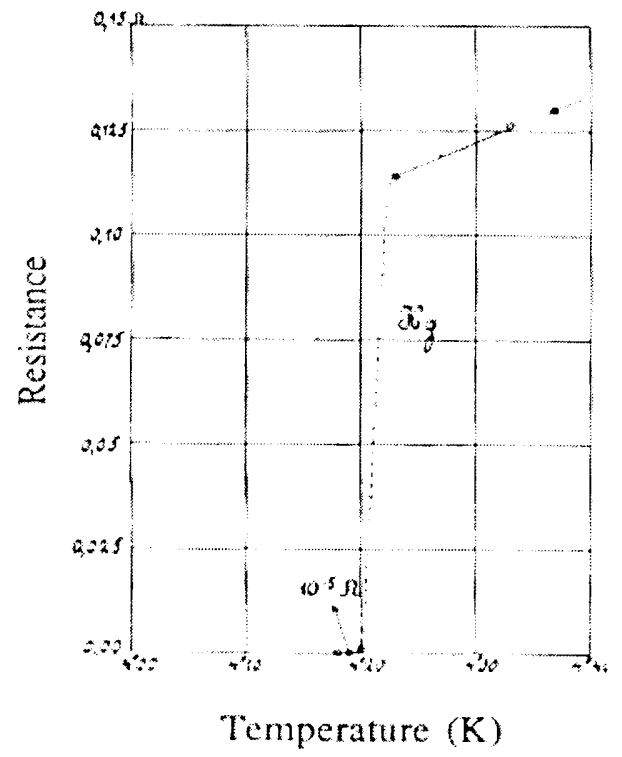

Figure 2.1 Resistance vs. Temperature of Mercury 
This spurred interest in scientists to better understand how matter behaves at extremely low temperatures. In 1933, Walter Meissner and Robert Ochsenfeld discovered that a superconducting material expels a magnetic field [5]. Faraday's Law tells us that a conductor in the presence of a time-varying magnetic field induces an electric field in the conductor, producing a current. However, in a superconductor, the induced currents produce magnetic fields that exactly cancel the field that would have otherwise penetrated the superconducting material. This absence of magnetic penetration, known as diamagnetism, is what is known as the Meissner Effect.

Over the next few years, a number of other metallic elements were found to be superconductors, such as Lead $(\mathrm{Pb})$ with a $T_{c}=7 \mathrm{~K}$ and Niobium $(\mathrm{Nb})$, with a $T_{c}=10 \mathrm{~K}$. In 1941 Niobium-Nitride (NbN) was found to superconduct at 16K. In 1953 VanadiumSilicon $\left(\mathrm{V}_{3} \mathrm{Si}\right)$ displayed superconductivity at $17.5 \mathrm{~K}$. At this point, researchers sought to apply this new technology to practical applications. Westinghouse scientists successfully displayed the viability of using superconductor material in a true world application when they developed the first commercial superconducting wire in 1962, an alloy of Niobium and Titanium (NbTi).

Up until 1986, the material with the highest recorded critical temperature had been that of a compound of Niobium and Germanium $\left(\mathrm{Nb}_{3} \mathrm{Ge}\right)$, with a $\mathrm{T}_{\mathrm{c}}=23.4 \mathrm{~K}$ [6]. This record was broken when scientists George Bednorz and Alex Müller at IBM's Research Laboratory in Rüschlikon, Switzerland, discovered superconductivity in a new material [7]. Their material, a brittle ceramic compound of Lanthanum, Barium, Copper and Oxygen $(\mathrm{LaBaCuO})$, superconducted at $35 \mathrm{~K}$ and became the first in a class of materials called "cuprates". This discovery was remarkable since a ceramic material had been found that was 
a superconductor, unlike most ceramics, which are poor conductors of electricity. It is with this discovery that the first "high" temperature superconductor was discovered. Within weeks of the discovery, research groups at the University of Tokyo fully identified the superconductor phase as $\mathrm{La}_{2-\mathrm{x}} \mathrm{Ba}_{\mathrm{x}} \mathrm{CuO}_{4 y}$. Subsequently, the substitution of Strontium ( $\mathrm{Sr}$ ) for La increased the $T_{c}$ to $40 \mathrm{~K}$. In 1987, Chu from the University of Houston first reported superconductivity in a new compound that substituted Yttrium $(\mathrm{Y})$ for $\mathrm{Sr}$ resulting in $\mathrm{YBaCuO}_{7-\mathrm{x}^{*}}$ This material superconducted at an incredible $92 \mathrm{~K}$, and pushed the $\mathrm{T}_{c}$ limit even further [8]. Now a material had been found that could be cooled to the temperature of liquid nitrogen and be superconducting. This sparked great interest in the scientific community to consider the possibility of developing novel electronics that would benefit from the superior performance of superconductors. The higher operating temperatures meant that the cost associated with cooling could be greatly reduced, as liquid nitrogen is both easier and cheaper to obtain than liquid helium type cooling systems. Since then, other materials have been synthesized that have critical temperatures as high as $140 \mathrm{~K}$. Figure 2.2 shows the trend of critical temperature with each discovered superconductor. 


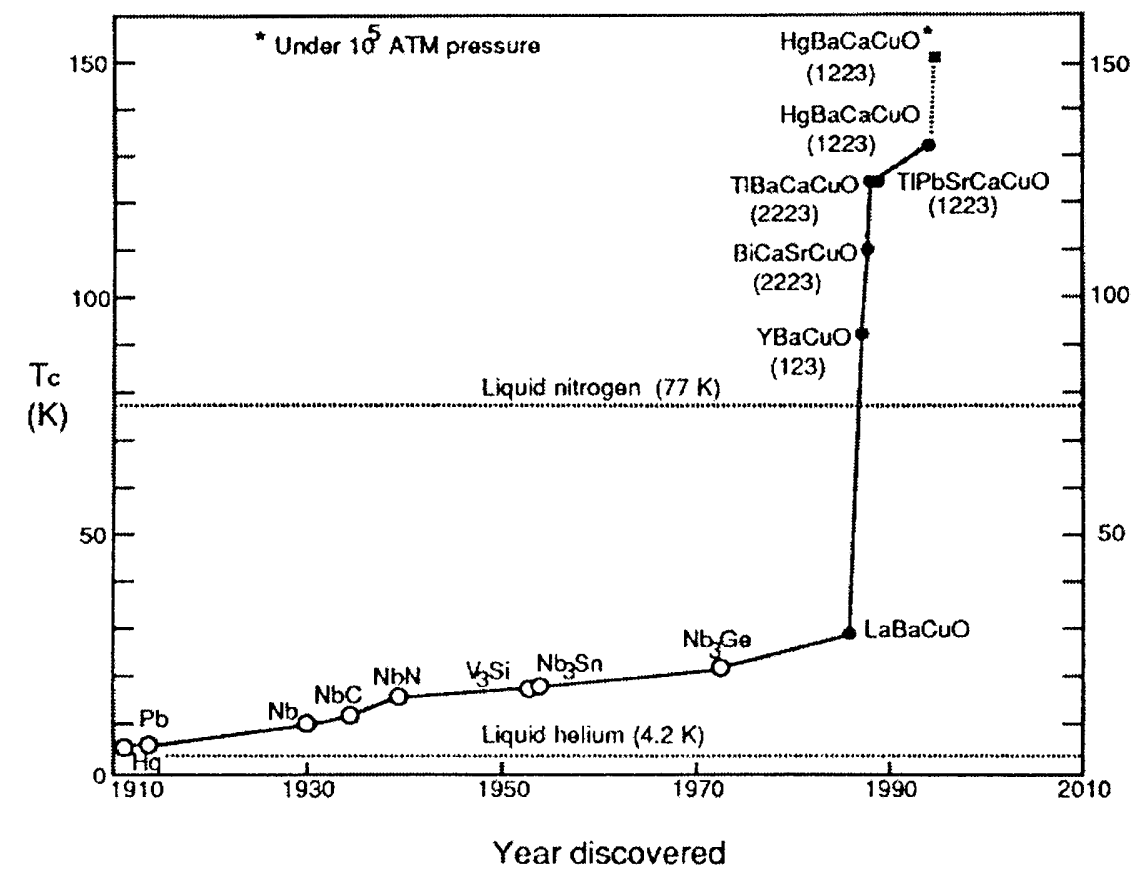

Figure 2.2 Superconductive materials and their year of discovery

\subsection{Low Temperature vs. High Temperature Superconductors}

Low temperature superconductors (LTS), those with $T_{c}<25 \mathrm{~K}$ are mostly comprised of single elements, such as $\mathrm{Pb}, \mathrm{Nb}, \mathrm{Al}$ and metallic alloys. Only 6 out of the 25 elements that are superconducting have $T_{c}<4.2 \mathrm{~K}$, ruling out the practical use of many of the singleelement superconductors. It is because for optimal performance superconductors' generally preferred operating temperature is one-half that the material's $T_{c}$ or below [9]. Hence with liquid helium refrigeration technology, early applications involved only materials with $T_{c} \geq$ 8K. As it was several years before high temperature superconductor (HTS) materials were discovered, quite a number of applications did develop for LTS use.

The first application of superconductors came with the use of $\mathrm{Nb}_{3} \mathrm{Sn}$ and then $\mathrm{NbTi}$ to make superconducting wires. Niobium $\left(\mathrm{T}_{\mathrm{c}}=9.2 \mathrm{~K}\right)$ has found wide use in electronic 
applications because of its chemical and mechanical stability. Other alloys and compounds of $\mathrm{Nb}$ such as $(\mathrm{NbN})$ and niobium titanium nitride $(\mathrm{NbTiN})$ have also been investigated for use in electronics. These LTS materials are used in two major areas. Large-scale applications usually involved the use of wires and conductor tapes to make coils for electrical machines. Small-scale applications involved use of thin films to fabricate RF circuits and other electronic components.

\subsubsection{Large Scale Applications}

The major large-scale applications have been in the following areas:

1. High field magnets for magnetic resonance imaging (MRI) and nuclear magnetic resonance spectroscopy (NMR).

2. Particle Accelerators for experimental physics.

3. In the separation of magnetic ore from an inhomogeneous slurry.

4. Electric power generators and transformers.

5. Power energy storage devices, such as superconducting magnetic energy storage system (SMES), that can be used for UPS backup and other power conditioning applications.

6. Magnets for levitation of transportation vehicles.

7. Motors for ship and submarine propulsion.

8. Magneto-hydrodynamic and fusion power systems. 
From the list above, the following illustrates some current examples of LTS technology in use. Particle Accelerators such as the superconducting Tevatron at the Fermi National Accelerator Laboratory has demonstrated that higher particle energies with a much lower power input are obtained when superconducting magnets are employed. High-energy particle research hinges on being able to accelerate sub-atomic particles to nearly the speed of light. Superconductor magnets make this possible. CERN, a consortium of several European nations, is currently developing a similar unit with its Large Hadron Collider (LHC) now under construction some details of which may be found in $[10,11]$.

MRI units that use superconducting magnets have been available for years and are present in many hospitals in North America. Systems that use superconducting magnets have greater sensitivity and obtain clearer, more detailed imagery. NMR spectrometers made with superconducting magnets, are able to achieve high fields with reduced size [12]. Several of which are available on the market today.

The US Navy already has plans to develop large horsepower (3000 - 25,000hp) motors that use LTS coils, for ship and submarine propulsion systems [13]. In Japan, research into magnetic levitation systems has led to the development of a fully operational Maglev test train running at 343-mph [14]. In all the above-mentioned applications, LTS materials, in particular NbTi and $\mathrm{Nb}_{3} \mathrm{Sn}$ have held their place over HTS due to 1) Easier preparation because of better malleability and ductility of the materials, 2) Ability to obtain higher current densities without loss of superconductivity, with excellent isotropy and 3) Also the ability to sustain higher magnetic fields before breakdown occurs.

Current research is enabling HTS materials to approach the performance of LTS in some the applications mentioned above. One major application where HTS has shown its 
superiority in both cost-effectiveness and performance is with HTS power transmission conductors. HTS cables have been developed that carry $2.4 \mathrm{kA}$ at $24 \mathrm{kV}$ with ease, exceeding the capability of normal conductors [15]. In fact, Detroit Edison is planning to install HTS cables in their main power grid, the first commercial application HTS cables.

\subsubsection{Small Scale Applications}

John Clarke loosely breaks down the small-scale applications into three main categories [16]. Those related to the detection of signals, the processing of analog signals and those dealing with digital signals as shown in figure 2.1.2. For each of these areas, LTS has performed quite well and has set the stage for HTS application development.

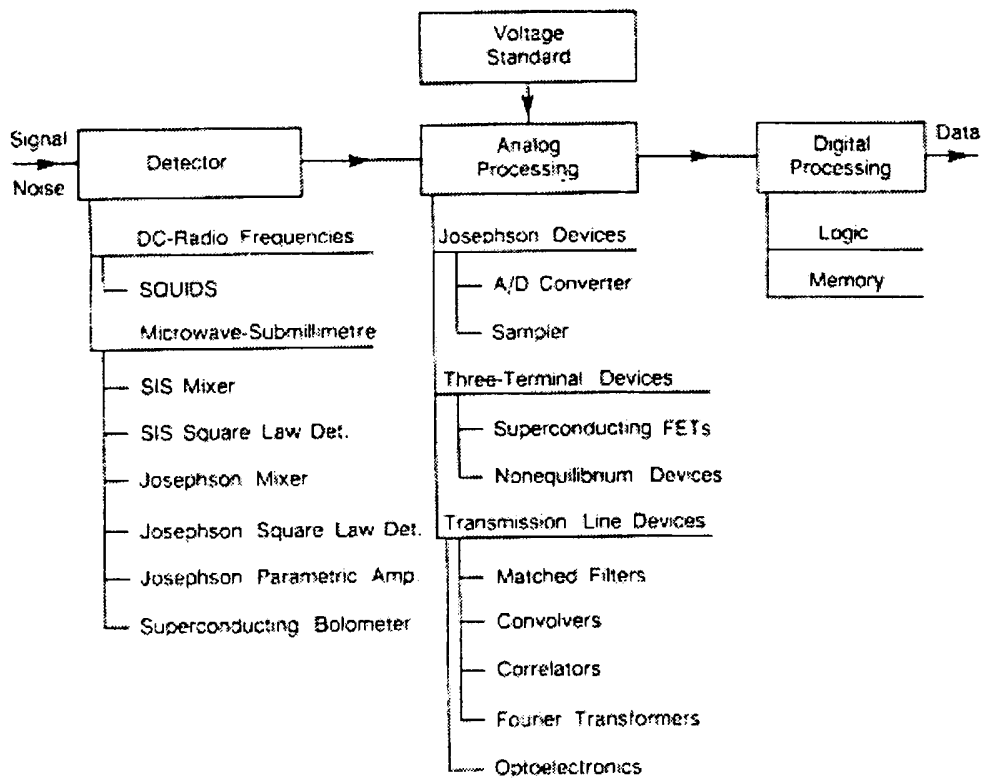

Figure 2.2.2 Small-scale applications of superconductors 
In 1963, Anderson experimentally verified the superconducting tunneling phenomena predicted by Brian Josephson the previous year $[17,18]$. This discovery led to the development of devices based on Josephson Junctions.

These devices depend on weak interactions that occur when two superconducting materials are in close proximity, separated only by a thin insulating layer. If a weak link exists, it is possible to have current flow across the junction by way of superconducting electrons tunneling through the insulating layer. The transition through the tunneling barrier introduces a phase difference $\delta$ in the current that is well defined up to a critical current $I_{o}$. Upon increasing current $I>I_{o}$, a voltage $V$ is developed across the junction. The current may be applied by adding a signal to the operating current or by placing the junction in a magnetic field. This voltage can switch its state in $\sim 3-5$ ps. This is attractive for digital circuits that could operate in excess of $100 \mathrm{GHz}$.

Devices such as the Superconducting Quantum Interference Devices (SQUIDs) developed in 1964 [19] are based on Josephson junction technology. SQUIDs are devices that can detect extremely small magnetic fields. Typical applications are in precision measurements of electrical currents and voltages, or in bio-magnetism measurements such as Magneto-cardiograms of the human heart (MCG) and Magneto-encephalography (MEG).

The sensitivity of SQUIDs is about 1 - $10 \mathrm{fT}$, for LTS SQUIDs and 10-100 fT, for HTS SQUIDs [16,20]. Clearly LTS have the advantage of higher sensitivities due to lower intrinsic noise from operating at lower temperatures.

Another important application developed first with LTS is Rapid Single Flux Quantum (RSFQ) logic [9]. Based on Josephson Junctions, RFSQ realizes logic functions by the storage of a single quantum of magnetic flux that is controlled by an applied current. 
The presence of this single flux quanta represents a logic " 1 " while the absence represents a logic "0". The switching speeds that can be achieved are extremely fast (>10 Ghz) and do so with much lower power consumption than traditional CMOS devices [2,9]. LTS RSFQ is applied in several areas, including A/D converters, D/A converters, digital SQUIDs, digital auto-correlators, and pseudo-random signal generators [9,16,21-23]. Research into enhanced fabrication technology, in the near micron or sub-micron range, shows the potential for ultra-fast digital switching, complex digital signal processing (DSP), as well as high end supercomputing.

In fact, the Hybrid Technology Multi-Threaded (HTMT) Architecture project led by an American consortium, has set plans to develop a supercomputer that would be two hundred and fifty times faster than today's fastest supercomputer. RSFQ superconducting circuits and other cryoelectronic components form the core of this computer's design [24]. John Rowell gives a review of the requirements for such a "petaflop" computer [25,28].

\subsubsection{RF Applications}

In the 1950s, RF applications began to be developed around LTS materials. The key interest, at the time, was in the development of microwave resonant cavities. $\mathrm{Pb}$ and $\mathrm{Nb}$ cavities were developed with extremely high Q-values, on the order of $10^{10}-10^{11}$ at liquid helium temperatures [26]. Other applications involving passive components were also investigated but because of the extremely low operating temperatures, saw limited use [26]. However since metallic LTS materials have the advantage of lower processing temperatures and easier fabrication. Oates and researchers at Lincoln Laboratory, MIT, currently use $\mathrm{Pb}$ 
in designing passive superconducting filters with ferrite tuning elements [27]. All of these applications are based on the use of thin film form of the material.

Since the discovery of high temperature superconductors (HTS), there has been great interest in utilizing the material to build novel electronic devices that can take advantage of the higher operating temperatures. Components such as resonators, filters, delay lines and patch antennas have been shown to perform significantly better when HTS material is used [29]. The surface resistance of superconductor films to RF signals is 10-100 times lower than that of normal conductors. This translates into lower insertion loss, lowered conductor losses, lower noise figures and the ability to design resonators/filters that have higher $\mathrm{Q}$ values and hence sharper filter skirts [29].

Such superconducting filters are presently being used in cellular communication systems to provide enhanced channel selectivity, increasing signal-to-noise ratio and hence better overall site performance $[29,30]$. The technology used in such applications is that of $\mathrm{YBaCu}_{3} \mathrm{O}_{7}(\mathrm{YBCO})$ and $\mathrm{Tl}_{2} \mathrm{Ba}_{2} \mathrm{CaCu}_{2} \mathrm{O}_{8}(\mathrm{TBCCO})$ thin films, patterned on $\mathrm{LaAlO}_{3}, \mathrm{MgO}$ and sapphire $\left(\mathrm{Al}_{2} \mathrm{O}_{3}\right)$ substrates $[1,29]$.

The other type of RF application was in active devices. Based on the Josephson junction, a number of devices such as flux-flow transistors, mixers, detectors, oscillators and parametric amplifiers have been made [31]. These applications, previously dominated by LTS, are now being replaced slowly with HTS technology.

For this thesis the application that will be explored is that of HTS resonators. HTS resonators have been successfully demonstrated by a number of groups [21,32-37]. For microwave frequencies, these resonators are made with a microstrip, stripline or co-planar waveguide configuration patterned on a suitable substrate. The choice of substrate material 
is important since HTS materials such as YBCO need to have a chemically inert, crystallographically compatible surface on which to grow. For high frequency applications, electronic properties such as effective dielectric constant and dielectric loss also are also of paramount importance. 


\subsection{History}

In 1955, Bardeen, Cooper, and Schrieffer developed the BCS theory, a microscopic theory of superconductivity based on interactions between electrons and the crystal lattice of the material [38]. When an electron moves in a crystal, a slight perturbation of the crystal lattice results from Coulomb attractive forces between the negatively charged electron and the positively charged ions of the crystal. If the perturbation occurs long enough, another electron in the vicinity will be affected. Under certain circumstances, this can result in a weak interaction between two electrons. This interaction may be such that the Coulombic repulsion normally experienced may be overcome and the electrons bind together to form a pair. Each electron in this "Cooper Pair" must have opposite magnetic spin in order to satisfy Pauli exclusion rules. BCS showed that a wave function could be constructed in which all electrons are paired. The electrons in the pair are "coherent" over a certain distance $\xi_{0}$, called the coherence length. This distance defines the size of the wave packet considered to be a result of the interaction between paired electrons. The coherence length also designates superconductors as being either of Type I or Type II variety. Type I superconductors are those that generally have large $\xi_{\mathrm{o}}$, on the order of a few thousand 
angstroms. For these, an applied magnetic field is completely excluded below a certain critical field $\mathbf{H}_{\mathbf{c}}$. Above this critical field, superconductivity is totally destroyed. For type II materials, two critical fields appear. When the first $\mathbf{H}_{\mathrm{c} 1}$ is exceeded, the superconductor goes into a mixed state. In this state, flux begins to penetrate the superconductor in the form of vortices, each with a circulating supercurrent. Upon further increase of applied field, the vortex density increases until at the second critical magnetic field $\mathbf{H}_{\mathrm{c} 2}$, superconductivity is destroyed. Because the second critical field is much greater than the first, this enables Type II superconductors to carry large currents before loss of superconductivity occurs. This critical current density, $J_{c}$, generally exceeds that of Type I materials. The coherence length in Type II superconductors is generally small, typically less than the penetration depth, which is discussed in the next section. For a more in-depth treatment of Type I and Type II superconductors, the reader is directed to $[9,39-42]$.

\subsection{Meissner Effect}

In 1933 Meissner and Ochsenfeld, observed two things about superconductors. First, when a magnetic field is applied to a superconductor, it is expelled. That is, no magnetic flux appeared to penetrate the material. Second, they observed that if a magnetic field was applied to a superconductor while in its normal state (i.e., $\mathrm{T}>\mathrm{T}_{\mathrm{c}}$ ) and the material was cooled to below $T_{c}$, then the magnetic field would be expelled. There is also a maximum field $H_{o}$, however, that once exceeded the superconductor becomes nonsuperconducting, as there is now penetration of magnetic flux [5]. This field is known as the 
thermo-dynamical critical field $H_{c}$. It has been shown empirically that $H_{c}$ had a temperature dependence that followed:

$$
H_{c}(T)=H_{c}(0)\left[1-\left(\frac{T}{T_{c}}\right)^{2}\right]
$$

where $H_{c}(0)$ is the critical field at $\mathrm{T}=0 \mathrm{~K}$.

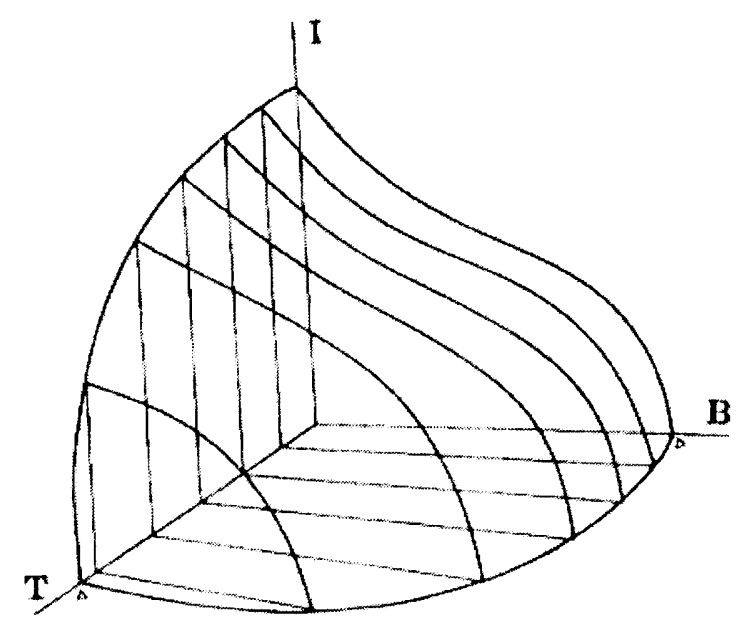

Figure $3.2 \quad$ I-B-T surface which within, superconductivity occurs

Consider a superconductor that is at $\mathrm{T}<\mathrm{T}_{\mathrm{c}}$. The force due to the acceleration of the carriers balances the force due to the electric field.

$$
q_{s} \mathbf{E}-m_{s} \frac{d \mathbf{v}}{d t}=0
$$


Where $m_{s}$ is the effective mass of the superconductive carriers, $\mathbf{v}$ is the drift velocity of the carriers, and $q_{s}$ the charge. The current density will then be:

$$
\mathrm{J}_{\mathrm{s}}=n_{s} q_{s} \mathbf{v}
$$

where $n_{s}$ is the number of superconductive carriers. Substituting (2) into (1) yields:

$$
\mathbf{E}=\Lambda \frac{d \mathbf{J}_{\mathrm{s}}}{d t}
$$

where $\Lambda$, the London constant is a phenomenological constant equal to:

$$
\Lambda=\frac{4 \pi \lambda_{L}^{2}}{c^{2}}=\frac{m_{s}}{n_{s} q_{s}^{2}}
$$

Where $c$ is the speed of light and $\lambda_{L}$ is the London penetration depth. Equation (3), denoted the first London equation, after F. and H. London [43] describes perfect conductivity since an electric field will accelerate the carriers $q_{s}$ instead of competing with a resistance to maintain their velocity as in normal conductors.

If the curl of (3) is taken and Faraday's law, $\nabla \times \mathbf{E}=-\mu \frac{d \mathbf{H}}{d t}$ is applied then: 


$$
\nabla \times \mathbf{E}=\Lambda \frac{d}{d t}\left[\nabla \times \mathbf{J}_{\mathrm{s}}\right]=-\mu \frac{d \mathbf{H}}{d t}
$$

and,

$$
\frac{d}{d t}\left[\nabla \times \mathbf{J}_{\mathrm{s}}+\mu \frac{\mathbf{H}}{\Lambda}\right]=0
$$

Application of Maxwell's equation (neglecting the displacement current), $\nabla \times \mathbf{H}=\mathrm{J}_{\mathrm{s}}$ to (6) yields:

$$
\frac{d}{d t}\left[\nabla^{2} \mathbf{H}-\frac{\mathbf{H}}{\lambda_{I}^{2}}\right]=0
$$

where the London penetration depth is defined as:

$$
\lambda_{\mathrm{L}}=\sqrt{\frac{\Lambda}{\mu}}=\sqrt{\frac{m_{s}}{\mu n_{s} q_{s}^{2}}}
$$

Equation (7) shows that if the magnetic field is initially zero inside a superconductor, and a magnetic field is then applied, there will be no resultant magnetic field. This explains the original observation of Meissner. However, if the field were non-zero to begin with, then (7) does not predict that there will be no net magnetic field. In fact, it suggests that magnetic flux would be trapped inside the superconductor. 
The London brothers explained this by prosing that not only was the time derivative of (7) equal to zero, but the argument as well. Hence, from (6) and (7):

$$
-\lambda_{L}^{2}\left[\nabla \times \mathbf{J}_{\mathbf{s}}\right]=\mathbf{H}
$$

and,

$$
\nabla^{2} \mathbf{H}-\frac{\mathbf{H}}{\lambda_{L}^{2}}=0
$$

Equation (9) is known as the second London equation and leads directly to Equation (10). In one dimension, (10) has the solution:

$$
\mathbf{H}(x)=\mathbf{H}(0) e^{-x / \lambda_{L}}
$$

Equation (11) shows that an applied magnetic field will decay exponentially inside a superconductor to a depth of $\lambda_{L}$, after which the magnetic flux $\mathbf{B}=0$. By rearrangement, it can be shown that the same applies to an electric field [44].

The penetration depth is an important parameter when considering superconducting thin films used in passive microwave circuits since it directly relates to the surface resistance $\mathrm{R}_{\mathrm{s}}$. With a time varying field, losses in the superconductor are quantified through its surface resistance $R_{s}$. From (8), we see that London Constant $\Lambda$, can be expressed in terms of the penetration depth. Considering an electric field with sinusoidal time-dependence and 
applying the first London equation, it can be shown that the penetration depth relates to the conductivity of the material and hence to surface resistance. This is explained in the next section with the use of the two-fluid model.

\subsection{Two-fluid Model}

Before the BCS theory, Gorter and Casimir $[45,46]$ developed the two-fluid model. This model is based on the assumption that a current flowing in a superconductor is due to two components, a normal component and a superconductive component. They showed that the total number of carriers $n=n_{s}+n_{n}$, the sum of superconducting and normal carriers and the ratios of each to the total number of carriers were related to the temperature as follows:

$$
\begin{aligned}
& \frac{n_{n}}{n}=\left(\frac{T}{T_{c}}\right)^{4} \\
& \frac{n_{s}}{n}=1-\left(\frac{T}{T_{c}}\right)^{4}
\end{aligned}
$$

Substituting (13) into (8) yields the temperature dependence of the penetration depth.

$$
\lambda_{L}(T)=\frac{\lambda_{L}(0)}{\sqrt{1-\left(\frac{T}{T_{i}}\right)^{4}}}
$$


The value of $\lambda_{L}$ at $T=0 \mathrm{~K}$ is defined as:

$$
\lambda_{L}(0)=\sqrt{\frac{m_{s}}{\mu n q_{s}^{2}}}
$$

\subsection{Surface Impedance}

The surface impedance $Z_{s}$ is defined as the impedance experienced by an electromagnetic plane wave, perpendicularly incident to the surface of a conductor. The mobile charge carriers within the material primarily determine the result of interactions of electromagnetic waves with conducting media. For both normal conductors and superconductors, this parameter is the electrical conductivity $\sigma$. From Ohm's law:

$$
\mathrm{J}=\sigma \mathrm{E}
$$

and Maxwell's equation in phasor form:

$$
\nabla \times \mathrm{E}=-j \omega \mu \mathrm{H}
$$

we can derive a wave equation that determines the penetration of electromagnetic fields into the conductor. If the curl of both sides of (17) are taken then:

$$
\nabla \times \nabla \times \mathbf{E}=-j \omega \mu \nabla \times \mathbf{H}
$$


Substituting,

$$
\nabla \times \mathbf{H}=(\sigma+\varpi \varepsilon) \mathbf{E}
$$

and noting that for good conductors, $\sigma>>\omega \varepsilon$, then,

$$
\nabla^{2} \mathbf{E}=\left[j \omega \mu \sigma-\mu \varepsilon \omega^{2}\right] \mathbf{E}=j \omega \mu \sigma \mathrm{E}
$$

Solutions of this equation are of the form,

$$
E_{i}=E_{0} e^{-\Gamma x}
$$

where $\Gamma$ is the propagation constant and is defined as ,

$$
\Gamma=\sqrt{j \omega \mu \sigma}=\frac{(1+j)}{\sqrt{2}} \sqrt{2 \pi f \mu \sigma}=\frac{(1+j)}{\delta}
$$

where

$$
\delta=\frac{1}{\sqrt{\pi j \mu \sigma}}
$$

Substituting (22) into (21), 


$$
E_{i}=E_{0} e^{-x / \delta} e^{-j / \delta}
$$

Equation (24) describes the behavior of the perpendicular component of the electric field on the surface of a plane conductor. It should be noted that similar forms of equation (20) might be obtained for both the magnetic field and the current density yielding similar forms (24). Thus, (24) tells us that the magnitude of the electric field (also magnetic field and current density, for that matter) decay exponentially within the conductor, where at a depth of $\delta$, they have decreased by about $63 \%$ of their value at the surface of the conductor. Hence it can be said that the fields have penetrated to a depth of $\delta$. This depth is known as the skin depth. It should be noted the London penetration depth $\lambda_{I}$ is frequency independent, unlike the skin depth $\delta$ in classical conductors, which has frequency dependence.

The surface impedance is then defined by the perpendicular electric field divided by the surface current density's transverse component.

$$
Z_{s}=\frac{E_{i}}{J_{s i}}
$$

where $E_{\tau}$ can be defined as,

$$
E_{\tau}=\frac{J_{0}}{\sigma}
$$


$J_{\kappa}$ is the total current flowing through a cross-section of unit width on the surface of the conductor. Recalling that $J_{\tau}$ has the form of (24), then $J_{s_{\tau}}$ can be found by summing the contributions of $J_{\tau}$ along the axis of penetration.

$$
J_{s \tau}=\int_{11}^{\infty} J_{i} d x=\int_{11}^{\infty} J_{10} e^{-x / \delta} e^{-j \times / \delta}=\frac{\delta}{(1+j)} J_{11}
$$

which is the limiting value. Thus $Z_{s}$ is,

$$
Z_{s}=\frac{J_{0}}{\sigma} \frac{(1+j)}{\delta J_{0}}=\frac{(1+j)}{\delta \sigma}
$$

Noting that the surface impedance has both real (resistive) and imaginary (inductive) parts then it can be expressed as,

$$
Z_{s}=R_{s}+j \omega L
$$

where $R_{s}$ is the resistance seen at the surface and $\omega L$ is the reactance seen internally within the conductor due to the fields penetrating the conductor. For normal conductors, $\sigma$ is real and thus,

$$
R_{r}=\omega L=\frac{1}{\delta \sigma}=\sqrt{\frac{\pi f \mu}{\sigma}}
$$


from (30) we see that the surface impedance is proportional to the square root of the frequency. This is an important difference from superconductors.

For superconductors, the difference lies in the conductivity parameter. From the two-fluid model, the current density $J_{\tilde{\tau}}$ has two components due to the superconductive and the normal carriers:

$$
J_{\tilde{\tau}}=J_{s}+J_{n}
$$

Also, the conductivity $\sigma$ has two components $\sigma_{\mathrm{s}}$ and $\sigma_{\mathrm{n}}$. The superconductive component of the conductivity $\sigma_{\mathrm{s}}$ can be found from equation (3), the first London equation. Expressed in phasor form,

$$
\mathbf{J}_{\mathrm{s}}=\frac{1}{j \omega \Lambda} \mathbf{E}=\sigma_{s} \mathbf{E}
$$

so,

$$
\sigma_{s}=\frac{1}{j \omega \Lambda}=\frac{1}{j \omega \mu \lambda_{L}}
$$

which shows that $\sigma_{s}$ is purely imaginary and does not contribute to any loss. For the normal component,

$$
\mathrm{J}_{\mathbf{n}}=n q\langle\mathbf{v}\rangle
$$


The average velocity is used since it assumed that the portion of carriers in the superconductor that are in a normal state, are in an excited state relative to the superconductive carriers. They are subject to multiple collisions and thus their net velocity can only be expressed as an average. Using Newton's second law, we can equate the forces due to motion with the force due to the electric field.

$$
m\left[\frac{d\langle\mathbf{v}\rangle}{d t}+\frac{\langle\mathbf{v}\rangle}{\tau}\right]=q \mathbf{E}
$$

where the second term accounts for that portion of the carriers undergoing collisions with a finite relaxation time $\tau$. Substitution of (34) into (35) yields,

$$
m\left[\frac{d}{d t}\left(\frac{\mathbf{J}_{\mathrm{n}}}{n q^{2}}\right)+\frac{\mathbf{J}_{\mathrm{n}}}{n q^{2} \tau}\right]=\mathbf{E}
$$

recalling that $\mathbf{J}_{\mathbf{n}}$ has sinusoidal time dependence,

$$
\begin{aligned}
& \mathrm{J}_{\mathbf{n}} \frac{m}{n q^{2}}\left[\frac{\tau j \omega+1}{\tau}\right]=\mathbf{E} \\
& \mathbf{J}_{\mathbf{n}}=\left[\frac{\tau}{\tau j \omega+1}\right] \frac{n q^{2}}{m} \mathbf{E}=\sigma_{n} \mathbf{E}
\end{aligned}
$$


We see that because of the sinusoidal time dependence, the normal state conductivity $\sigma_{\mathrm{n}}$ has a complex component:

$$
\sigma_{n}=\left(\frac{n q^{2} \tau}{m}\right) \frac{1-j \omega \tau}{\tau^{2} \omega^{2}+1}=\left(\frac{n q^{2} \tau}{m}\right)\left[\frac{1}{\tau^{2} \omega^{2}+1}-\frac{j \omega \tau}{\tau^{2} \omega^{2}+1}\right]
$$

Hence the total conductivity in a superconductive conductor is,

$$
\sigma=\sigma_{n}+\sigma_{s}=\left(\frac{n q^{2} \tau}{m}\right)\left[\frac{1}{\tau^{2} \omega^{2}+1}-\frac{j \omega \tau}{\tau^{2} \omega^{2}+1}\right]+\frac{1}{j \omega \mu \lambda_{L}}
$$

For frequencies below $10^{11} \mathrm{~Hz}$, which is usually the case for microwave applications, $\omega \tau<<1$, hence (39) can be simplified to,

$$
\sigma=\sigma_{1}-j \sigma_{2}=\frac{n q^{2} \tau}{m}-j \frac{1}{\omega \mu \lambda_{\mathrm{L}}}
$$

For the surface impedance of a superconductive plane conductor,

$$
Z_{s}=\sqrt{\frac{j 2 \pi f \mu}{\sigma_{1}-j \sigma_{2}}}=\sqrt{\frac{2 \pi f \mu}{\sigma_{1}+\frac{\sigma_{2}}{j}}}=j \sqrt{\frac{2 \pi f \mu}{\sigma_{2}}}\left(1+\frac{j \sigma_{1}}{\sigma_{2}}\right)^{-1 / 2}
$$


Noting that because of the extremely small value of $\tau, \sigma_{1}<<\sigma_{2}$ and (41) may be approximated by a binomial expansion such that,

$$
Z_{s}=j \sqrt{\frac{2 \pi f \mu}{\sigma_{2}}}\left(1-j \frac{\sigma_{1}}{2 \sigma_{2}}\right)=\sqrt{\frac{2 \pi f \mu}{\sigma_{2}}}\left(\frac{\sigma_{1}}{2 \sigma_{2}}+j\right)
$$

Which after substitution of (40) yields,

$$
Z_{s}=R_{s}+j X_{s}=\frac{2 \pi^{2} f^{2} \mu^{2} \lambda_{\mathrm{I}}^{2} n q^{2} \tau}{m}+j \omega \mu \lambda_{L}
$$

The real component $R_{s}$ contributes the loss and differs from the normal conductor case in that it is proportional to the square of the frequency. The reactance term in (43) is inductive and is know as the kinetic inductance as it reflects the kinetic energy of the superconductive carriers. This reactance is significantly greater than $R_{s}$ and dominates the surface impedance at microwave frequencies. The graph below shows how the surface resistance of the $\mathrm{HTS}$ material $\mathrm{YBa}_{2} \mathrm{Cu}_{3} \mathrm{O}_{7}$ compares with that of copper at $77 \mathrm{~K}$. 


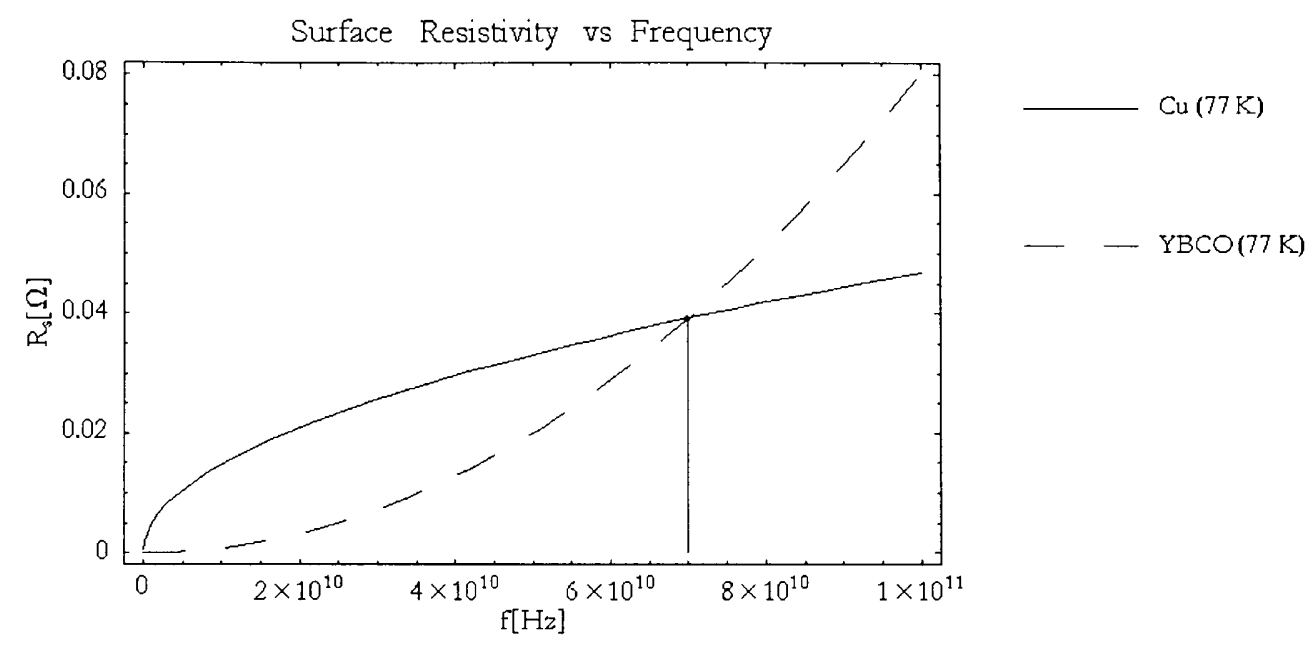

Figure $3.4 \quad$ Surface resistivity of Copper and YBCO at 77K

This shows that at a frequency of around $70 \mathrm{Ghz}$, the surface resistance of $\mathrm{Cu}$ at $77 \mathrm{~K}$ becomes less than that of $\mathrm{YBa}_{2} \mathrm{Cu}_{3} \mathrm{O}_{7}$, illustrating the advantage of the HTS material at frequencies below. It should also be mentioned that when used in thin film electronics, factors that influence the surface resistivity include thickness of the film and the temperature of operation. As the figure below shows, the variations in the impedance occur mainly at temperatures close to $T_{c}$. Below $T_{c} / 2$, there is little variation, making this temperature a popular operating point for most devices. 


\subsection{Substrates}

In order to realize HTS thin film circuits/devices, the material has to be deposited on a suitable substrate. For this thesis, the HTS material of interest is the compound $\mathrm{YBa}_{2} \mathrm{Cu}_{3} \mathrm{O}_{7-\mathrm{x}}(\mathrm{YBCO})$. The molecular structure is shown in the figure below.

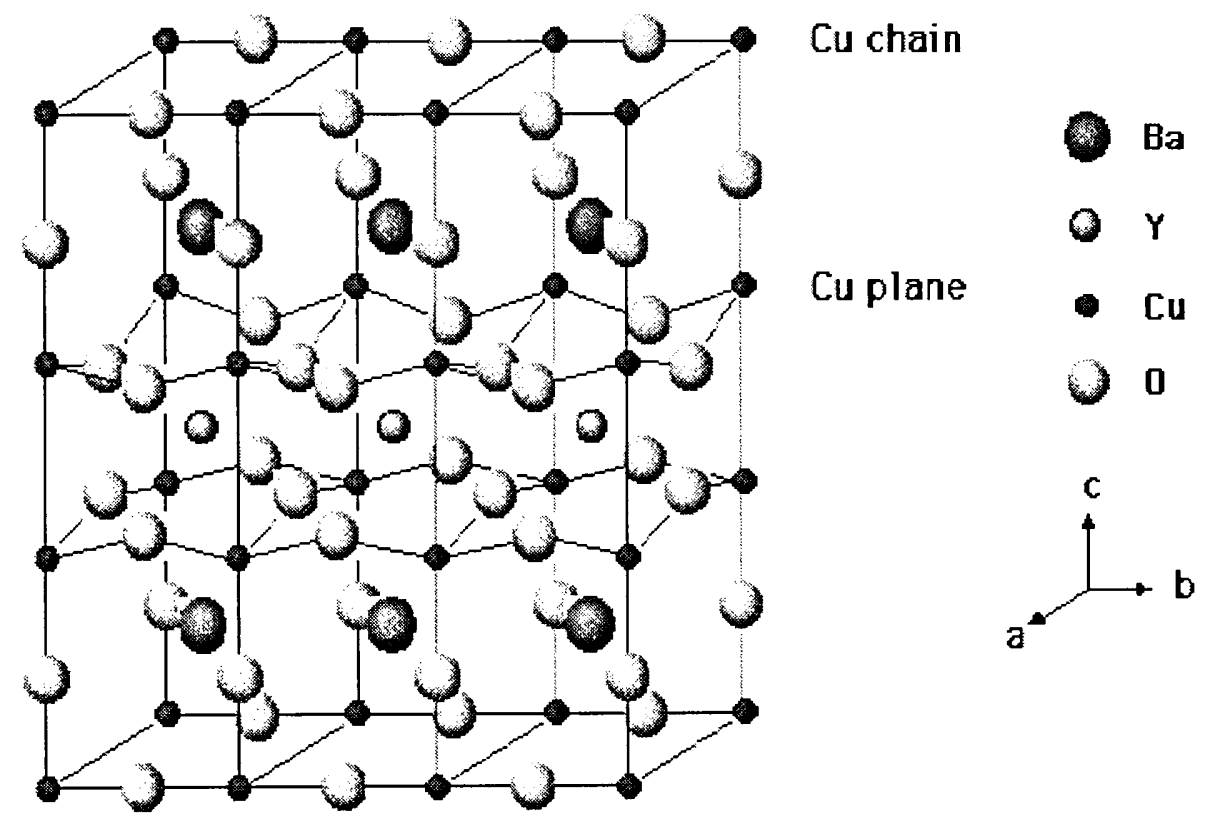

Figure 4.1 YBCO structure with $\mathrm{a}=3.821, \mathrm{~b}=3.885, \mathrm{c}=11.676 \AA$ 
YBCO is has two known phases that are related to the oxygen content $x$. For low oxygen content $(0.6<x<1)$, the result is compound that has a tetragonal crystal structure. This form is either non-superconducting or superconducts at a very low temperature. For $x$ $<0.6$, the compound forms in an orthorhombic structure and superconducts at temperatures of about $92 \mathrm{~K}$.

For most RF applications, YBCO is deposited with the c-axis perpendicular to the substrate. With reference to the above figure, c-axis orientation means that the copper oxygen planes are parallel with the substrate. For RF and microwave thin film applications, c-axis oriented films are preferred since the coherence length $\xi_{0}$ is longer, typically about 14 $\AA$, within $\mathrm{Cu}-\mathrm{O}$ planes than the $4 \AA$ perpendicular to them. This means that superconducting pairs have a longer range of order, parallel to the $\mathrm{Cu}-\mathrm{O}$ planes translating into enhanced current flow. The requirement of c-axis orientation also means that there must be a high degree of alignment of the unit cells on the substrate. This condition is known as epitaxy.

In order to realize an epitaxial relationship between the substrate and YBCO, the substrate itself must have similar a-b lattice parameters. A close lattice match translates into less stress/strain between substrate and the superconductor film. Minimizations of stress/strain results in higher quality films with less structural defects and hence better current transport properties.

In addition to the requirement of close lattice match, substrates must also be chemically inert. This requirement ensures that there is no inter-diffusion of chemical species between substrate and superconductor and that no chemical reactions occur that may destroy or change the structure and quality of the superconductor. 
Thermal stability of the substrate is also required such that crystallographic changes that may occur at elevated deposition temperatures and disrupt the growth of the superconductor are prevented. In addition, the thermal expansion properties should be close to that of YBCO since deposition usually occurs at elevated temperatures,

Finally, for RF and microwave applications, the substrate used should have good high frequency properties such as low dielectric loss and somewhat high dielectric constant. A high dielectric constant material allows feature sizes to be reduced, enabling better miniaturization. HTS materials have the advantage of better performance, when miniaturized, over normal conductors due to their lower surface resistance. Also, chemicals used in the processing of thin film circuits should be inert with respect to the substrate.

A variety of substrates have been used to deposit YBCO. They can generally be divided into three categories. (1) Those belonging to the perovskite family. (2) Nonperovskites that satisfy the conditions mentioned in section 4.1, and (3) those that do not satisfy the conditions and need further modification to allow YBCO growth.

The substrates that are classified as perovskites, derive their name from a specific mineral known as "perovskite". They are compounds that combine metallic elements with non-metals such as oxygen, and have a specific atomic arrangement. In their ideal form, perovskites, which are generally described by the chemical formula $\mathrm{ABO}_{3}$, consist of cubes made up of distinct chemical elements $(A, B$, and $O)$ that are present in the ratio of 1:1:3. The $\mathrm{A}$ and $\mathrm{B}$ atoms are metallic cations and the $\mathrm{O}$ atoms are non-metallic oxygen anions. YBCO has been shown to superconduct when deposited directly on substrates of this family, including $\mathrm{SrTiO}_{3}$ [47], $\mathrm{LaAlO}_{3}$ [48], $\mathrm{LaGaO}_{3}$ [49], $\mathrm{NdGaO}_{3}$ [50], $\mathrm{YAlO}_{3}$ [51]. These compounds also possess pseudo-cubic crystal structures like YBCO, with the exception of 
$\mathrm{SrTiO}_{3}$, which is a cubic crystal. The YBCO compound is itself a member of the perovskite family and has the advantage of being well lattice-matched to those perovskite substrates.

Substrates of the non-perovskite type that have been shown to be suitable for YBCO deposition include Magnesium Oxide, $\mathrm{MgO}$ [52] and Yttria-stabilized Zirconia [53]. Both of these materials have a cubic structure, however, their lattice constants do not match up as well with YBCO as the perovskites do. However, good quality YBCO films have been demonstrated on these substrates. A table of some popular substrates and their relationship with YBCO are shown in Table 4.1.

Substrates of the third category are required to have an additional layer of material grown on them such that the proper conditions for YBCO deposition are met. Two such substrates that have widely been used are Sapphire $\left(\mathrm{Al}_{2} \mathrm{O}_{3}\right)$ and Silicon $(\mathrm{Si})$. Sapphire when used without an intermediate layer out-diffuses $\mathrm{Al}$ into the YBCO during deposition. This renders the YBCO film useless. It has been found that a thin layer of $\mathrm{CeO}_{2}[1]$ buffers the $\mathrm{Al}_{2} \mathrm{O}_{3}$ and prevents any adverse reactions. There is great interest in buffering silicon with a variety of materials for different applications. As this is the subject of this thesis, the discussion on buffered silicon technology will be expanded in the next section.

\begin{tabular}{|l|l|l|l|l|}
\hline Substrate & $\begin{array}{l}\text { Crystal } \\
\text { Structure }\end{array}$ & $\begin{array}{l}\text { \% Lattice } \\
\text { mismatch with } \\
\text { YBCO }\end{array}$ & $\begin{array}{l}\text { Thermal Expansion } \\
\text { coefficient } \alpha\left(10^{-6} /{ }^{\circ} \mathrm{C}\right)\end{array}$ & $\begin{array}{l}\text { Relative } \\
\text { permittivity } \\
\varepsilon_{\mathrm{r}}\end{array}$ \\
\hline $\mathrm{LaAlO}_{3}$ & Rhombohedral & -0.7 & 10.0 & 24.5 \\
\hline $\mathrm{YSZ}$ & Cubic & -7 & 11.4 & 27 \\
\hline $\mathrm{MgO}$ & Cubic & 9 & 8.0 & 10 \\
\hline $\mathrm{SrTiO}_{3}$ & Cubic & -0.8 & 9.4 & $\sim 300$ \\
\hline $\mathrm{NdGaO}_{3}$ & Orthorhombic & 0.4 & 11.0 & 23 \\
\hline
\end{tabular}

Table 4.1 Popular substrates and their characteristics 


\subsection{Buffered Silicon}

Since the discovery of superconductors with critical temperatures, $T_{c}>77 \mathrm{~K}$, there has been interest in integrating these materials with existing silicon devices [54]. Devices such as high electron mobility transistors (HEMT), hetero-junction bipolar transistors (HBT) and some CMOS digital components, have shown to have enhanced operability at low temperatures. [55]. Since at this temperature most HTS materials are superconducting, there is a possibility of creating some novel silicon devices that also could benefit from the superior properties of superconductors. The zero resistance and high current density properties of superconductors make them ideal candidates for packaging and interconnect material in giga-scale integration applications [56]. Contacts and interconnects would have lower intrinsic delays and also the dimensions of the device could be minimized.

In making hybrid devices using YBCO and silicon, the need for an interfacial layer between the substrate surface and the growing film arises. This layer prevents the interdiffusion of atoms between substrate and film and hence prohibits any chemical reactions at the substrate surface with species of the deposited film. It has been shown that without a layer, $\mathrm{Ba}$ atoms out-diffuse from the $\mathrm{YBCO}$ and react with $\mathrm{Si}$ to form $\mathrm{BaSiO}_{4}$ [57]. This "buffer" layer should also be thermally stable, allowing the growing film to nucleate without undergoing any structural disturbance from possible temperature related phase transitions of the buffer layer. As mentioned in the previous section, compatibility with the substrate's unit cell size and thermal expansion properties are also necessary for high quality buffer layer growth. Similar compatibility with YBCO must exist to ensure its growth is of high quality by the reduction of mechanical stress, which arises from differences in thermal expansion coefficients of YBCO and Si. 
Silicon has diamond lattice with a cubic primitive unit cell. This unit cell has a lattice constant $a_{0}=5.43 \AA$. The crystal has a thermal expansion coefficient of $3.8 \times 10^{-6} /{ }^{\circ} \mathrm{C}$ and a relative dielectric constant $\varepsilon_{\mathrm{r}}=11.4$. It is attractive as a substrate for three main reasons. These are:

1. Its relatively lower cost than other substrate materials.

2. There is a possibility of creating hybrid microelectronics and combining them with existing silicon technology.

3. The techniques of silicon processing are well established in the semiconductor industry.

The pioneering work of Myoren et al in using Zirconium Oxide $\left(\mathrm{ZrO}_{2}\right)$ [58] as a buffer layer for YBCO on Si, led to many researchers to study the use of different insulating compounds as buffer layer material. Harada et al first reported the use of Magnesium Oxide $(\mathrm{MgO})$ as a suitable buffer layer [59]. Strontium Titanate $\left(\mathrm{SrTiO}_{3}\right)$ was shown by Mori and Ishiwara [60] to also be suitable. Inoue et al showed that Cerium Oxide $\left(\mathrm{CeO}_{2}\right)$ was also an excellent candidate [61]. Other materials such as Yttrium Oxide $\left(\mathrm{Y}_{2} \mathrm{O}_{3}\right), \mathrm{BaTiO}_{3} / \mathrm{MgAl}_{2} \mathrm{O}_{4}$ and $\mathrm{SrTiO}_{3} / \mathrm{MgAl}_{2} \mathrm{O}_{4}$ have been used as buffer layer between $\mathrm{YBCO}$ and $\mathrm{Si}$ [62-64].

The choice of buffer layer material is important when considering the type of application that the final hetero-structure is to be used for. In this work the scope of application is passive microwave resonators. For these applications, dielectric properties such as relative permittivity $\varepsilon_{\mathrm{r}}$ and dielectric loss factor $\tan \delta$ of the buffer layer material may 
become important depending on its thickness. Resistance to processing chemicals during resonator fabrication is also important

For this work, the substrate choice was silicon $(\mathrm{Si})$ with (100) orientation, i.e. the surface of the wafer represents the (100) face of a cubic unit cell. The superconducting material to be grown, in thin film form is a ceramic cuprate superconductor, $\mathrm{YBa}_{2} \mathrm{Cu}_{3} \mathrm{O}_{7}$, (also know as "YBCO” or "123").

Researchers at the Future Aerospace Science and Technology Center (FAST Center) at FIU have previously studied the use of materials such as $\mathrm{MgO}$ [65] and $\mathrm{ZrO}_{2}$ [66] as buffer layers on Si, for YBCO thin film electronic application. However, some properties of these materials prevented the growth of high quality superconducting YBCO. These were:

1. $M g O$ : High affinity for water vapor which destroys film quality.

2. $\mathrm{ZrO}_{2}$ : non-cubic unit cell resulting in poor lattice mismatch with silicon substrate

As a solution, the material proposed for buffer layers on $\mathrm{Si}$ is Yttria-stabilized Zirconia (YSZ, $\left.\left(\mathrm{Y}_{2} \mathrm{O}_{3}\right)_{\mathrm{x}}\left(\mathrm{ZrO}_{2}\right)_{1-\mathrm{x}}\right)$. This material consists of Zirconia with a certain mole percentage $(x)$ of Yttria $\left(\mathrm{Y}_{2} \mathrm{O}_{3}\right)$, enough to make a stable compound with a single cubic phase. Normal Zirconia experienced phase transitions from monoclinic $\rightarrow$ tetragonal $\rightarrow$ cubic depending on the growth temperature, with the tetragonal phase forming at $600^{\circ} \mathrm{C}$ and the cubic phase forming at $900^{\circ} \mathrm{C}$. The result is that Zirconia films deposited on Si would not grow uniformly and possessed mixed phases. By being stable in the cubic phase, YSZ is better matched for growth on the (100) Si and hence forms more homogenous films when grown on Si as compared to normal Zirconia. YSZ has a melting point of $2727^{\circ} \mathrm{C}$, has a 
refractive index on $2.12-2.22$ at a wavelength of $0.4-1.11 \mu \mathrm{m}$ and a high oxygen diffusion coefficient at temperatures above $300^{\circ} \mathrm{C}$. It has a lattice constant of $5.09 \mathrm{~nm}$ and a dielectric constant of 27. YSZ is virtually inert to processing chemicals such as etchants used in the fabrication of thin film components.

\subsection{YSZ Buffered Silicon}

YSZ thin films have been deposited on Si (100) substrates by a variety of methods such as electron-beam evaporation [67-69], laser ablation [70-72] and reactive sputtering [7378]. Lubig et al produced epitaxial (001) YSZ buffer layers on (100) Si by electron-beam evaporation from $\left(\mathrm{Y}_{2} \mathrm{O}_{3}\right)_{0.06}\left(\mathrm{ZrO}_{2}\right)_{10.94}$ pellets [67]. Their $\mathrm{Si}$ substrates were (100) oriented $\mathrm{p}$ type with a native oxide of several nanometers in thickness. They report that the YSZ films were highly oriented in spite of a native amorphous $\mathrm{SiO}_{2}$ layer present on the substrate. Bardal et al also produced epitaxial YSZ films on (100) Si substrates by way of electronbeam evaporation. However, they determined that although it is possible to grow epitaxial YSZ on oxidized Si, the thickness of the native oxide plays a great role in the growth process. They observed that with a $1.7 \mathrm{~nm}$ thick native oxide, highly (001) oriented YSZ films grew with grains having significant in-plane misalignment. It was observed that the $\mathrm{SiO}_{2}$ layer experienced some re-growth and was $4 \mathrm{~nm}$ thick after deposition, as measured by high-resolution transmission microscopy (HRTEM) [68]. Mathée et al concur with this result except that they found in growing the YSZ film, if the oxygen pressure is kept very low $\left(<7.5 \times 10^{-7}\right.$ Torr $)$ during the first $10 \AA$ of growth, the native oxide is significantly reduced by the following reaction [54]: 


$$
\mathrm{Zr}+2 \mathrm{SiO}_{2} \rightarrow \mathrm{ZrO}_{2}+2 \mathrm{SiO}
$$

This occurs because at the elevated deposition temperatures in high vacuum, there is some disassociation of the amorphous $\mathrm{SiO}_{2}$ layer from sections of the substrate [79]. This provides nucleation centers for $\mathrm{Zr}$ to react due to its high affinity for oxygen. The notion that $\mathrm{Zr}$ metal is present to react is supported by the fact that at low oxygen pressure, the evaporation of the YSZ pellets yields species deficient in oxygen and some completely depleted of oxygen.

Boikov et al made similar reports but used pulsed laser deposition to grow preferentially oriented (100) YSZ films on (100) Si. They too used oxidized Si substrates and used the process of initially having a low oxygen pressure during the first few angstroms of growth. YBCO grown on top of the YSZ/Si structure showed good (001) texture although not completely epitaxial [70]. Fenner et al present an interesting study of the interfacial reactions between YSZ and $\mathrm{Si}$ [71]. They examined reactions at the interfaces of laser ablated YSZ films on Si substrates with and without and oxide layer. They found that it is possible to obtain good YSZ films in both cases, with the exception of slightly better results on the non-oxidized Si. Fork et al proved this by producing high quality YSZ films on Si substrates that had their native oxide removed and its surface terminated instead by Hydrogen [72].

Deposition by sputtering is well known to be a simple and reproducible method of growing thin films. Commonly used in the microelectronics industry for coating of such 
things as hard disk platters, CD's and DVDs, and other tasks such as contact metallization, sputtering provides several advantages over other deposition methods. These are:

1. More control over process parameters.

2. Better chance of repeatability of resulting film characteristics

3. High deposition rates of single elements, especially metals.

4. Scaling to larger deposition areas is easier.

5. Uniform and smooth films may be obtained over large areas (6 inch wafers and greater).

Laser deposition suffers from the tendency of the growing film to form with droplets, relatively large conglomerates of the deposited material that lead to films having rough surfaces. Electron-beam evaporation suffers from films tending to sometimes grow with non-uniform thickness. However, research is being carried out to improve the laser and evaporation processes. Sputtering is therefore our method of choice for depositing YSZ thin films on Si.

Three types of sputtering system configurations have been used to deposit YSZ on Si. Two of these methods have been well used; RF Magnetron and DC Magnetron Sputtering. The third, Pulsed DC Magnetron (PDC) sputtering has been used in the coatings industry for the deposition of $\mathrm{Al}_{2} \mathrm{O}_{3}, \mathrm{TiO}_{2}$ and $\mathrm{SiO}_{2}$ [80-82]. However the PDC method has not been applied to the deposition of YSZ buffer layers for YBCO films on Si. This method is explored in this thesis. 
Hung et al report dominantly (100) oriented YSZ films grown on (100) Si by RF magnetron sputtering. They used a stoichiometric YSZ target and sputtered in an atmosphere of $94 \% \mathrm{Ar}$ and $6 \% \mathrm{O}_{2}$ at a total pressure of $100 \mathrm{~m}$ Torr. With sputtering power of $150 \mathrm{~W}$, the deposition rate was $4.16 \mathrm{~nm} / \mathrm{min}$ with the sample $44 \mathrm{~mm}$ away from the target. (100) films were obtained with substrate temperatures of $700^{\circ} \mathrm{C}$. They too observe a re-growth of a $\mathrm{SiO}_{2}$ layer after deposition on previously oxide-free $\mathrm{Si}$ substrates. They surmise that this is the reason they did not obtain fully epitaxial YBCO films grown on the $\mathrm{YSZ} / \mathrm{Si}[77]$.

Horita et al also sputtered YSZ on Si. They report DC magnetron reactive sputtering of a $98 \mathrm{~mm} \mathrm{Zr}$ target on which six $10 \mathrm{~mm}^{2} \mathrm{Y}$ chips arranged circularly. Their operating conditions were $7 \mathrm{mTorr}$ of $92 \% \mathrm{Ar}$ and $8 \% \mathrm{O}_{2}$ with a substrate temperature of $800{ }^{\circ} \mathrm{C}$. They used oxide-free Si substrates that were subsequently thermally oxidized before deposition of YSZ. As with the electron-beam researchers, they too determine that it is necessary to have a thin oxide layer present in order to obtain high quality epitaxial YSZ films. The films produced were (100) oriented $\left(\mathrm{Y}_{2} \mathrm{O}_{3}\right)_{1.118}\left(\mathrm{ZrO}_{2}\right)_{0.92}$ films [73].

Schmidt et al studied the dependence of oxygen pressure on the quality of sputtered YSZ films. The sputtered YSZ films on Si using $50 \mathrm{~W} \mathrm{RF}$ power and an 8-mole \% YSZ target. The substrate temperature was $800^{\circ} \mathrm{C}$ and the target distance was $10 \mathrm{~mm}$. They used $15 \mathrm{mTorr}$ of Ar with different $\mathrm{O}_{2}$ pressures and obtained deposition rates of $2 \mathrm{~nm} / \mathrm{min}$. They found that good (100) YSZ films were obtained with no oxygen pressure, with the quality of the films decreasing as the oxygen pressure increased to a maximum after which film quality again increased. They determined that after the maximum, higher working pressures reduced the adverse effect of the oxygen due to a decrease in the average mean 
free path of $\mathrm{O}_{2}$ ions. They also found that by delaying the injection of oxygen into the deposition chamber by $5-15 \mathrm{~s}$, also helped to produce better quality films [78].

Amor et al reported $\mathrm{RF}$ magnetron sputtering from a pure $\mathrm{ZrO}_{2}$ target onto glass slides. They studied the dependencies of deposition rate and total sputtering pressure on the quality of the resulting films. They found that a high deposition rate was necessary to obtain compact non-porous films. The also found that an increase of total pressure resulted in not only a decrease in deposition rate but also an increase in random orientation of the growing film. A low sputtering pressure results in an inadequate amount of Ar ions to sustain a stable deposition rate. Thus they suggest that there is an optimum deposition rate of about 7.5 mTorr that ensures high deposition rates and minimal randomness in the resulting film. Interestingly, compared to the results of Schmidt et al, Amor found that if no oxygen is used and high RF powers are used to increase the deposition rate, there is preferential sputtering of $\mathrm{Zr}$ from the composite target leading to films that are deficient in oxygen [80].

All the sputtering experiments described use the on-axis geometry. That is, the target is positioned on the axis normal to the substrate. It has been reported by Tsai et al that using off-axis geometry can reduce effects from plasma bombardment on the growing film. Off-axis means that the target is now oriented $90^{\circ}$ to the substrate. They R.F. sputtered a 10-mole \% YSZ target, $50 \mathrm{~mm}$ in diameter, in a 49 mTorr mixture of $75 \% \mathrm{Ar}$ and $25 \% \mathrm{O}_{2}$. Substrate temperature was $750{ }^{\circ} \mathrm{C}$. They show that higher quality films almost $100 \%$ oriented are obtained on $\mathrm{Si}$ substrates using the off-axis method [76]. However, the disadvantage of off-axis geometry is that deposition rates are extremely low.

The variety of results as led to questions regarding the dependencies of YSZ growth mechanism. It is clear that deposition rate, oxygen partial pressure, substrate temperature, 
and the presence of native oxides and re-grown oxides strongly influence the structural and interfacial properties of YSZ/Si heterostructures. The YBCO/YSZ interface has also been extensively investigated [84-85].

In this thesis, the goal is to evaluate RF and PDC magnetron sputtered YSZ films to obtain reliable process parameters such that good quality YSZ buffer layers may be reproducibly deposited on (100) Si. 


\subsection{Glow Discharges}

The techniques of sputtering have been widely developed since the discovery of the method in 1852 by Sir W. R. Grove. In sputter deposition, energetic particles bombard a target surface with sufficient energy such that, through momentum transfer, one or more atoms are ejected or sputtered from the target. The energetic particles are usually ions from an inert gas such as Ar or $\mathrm{Kr}$. Ar is used more frequently because of its relative abundance and low cost.

The process of generating ions and supplying them with enough energy for bombardment is a result of a phenomenon known as a Glow Discharge. A Glow Discharge can be created by applying a potential (hundreds to a few thousand volts DC or RF) between two electrodes in a gas enclosed in some container. Under appropriate gas pressure and applied voltage, electrons accelerated by the electric field, may gain enough kinetic energy to ionize the At atoms through an inelastic collision process known as electron-impact ionization [113]. In this process a primary electron removes an electron from the atom producing a positive ion and two electrons: 


$$
\mathrm{e}^{+}+\mathrm{Ar} \rightarrow 2 \mathrm{e}^{-}+\mathrm{Ar}^{+}
$$

The two electrons then continue under the influence of the electric field, to initiate more collisions and hence more ionizations. Other species, such as photons and ions themselves can also be responsible for ionization $[113,90]$. The degree of ionization depends on the kinetic energy of the electrons/ions and the ionization potential of the gas atoms. The ionization potential defines the minimum energy required to remove an electron completely from the atom. Depending on the gas density, the particles that influence ionizations lose some of their kinetic energy through collisions with themselves. As a result, not all of the gas molecules are ionized. Of those that do not undergo ionization, some stay neutral while others enter into an excited state. The excited state results from an inelastic collision that imparts enough energy to a bound electron such that it enters a higher energy level. This excited state is not stable and eventually the electron falls back to its original level and the lost energy is emitted as a photon. This is the reason for the characteristic "glow" feature of glow discharges. The combination of ionized particles, electrons, and neutral atoms is called plasma and forms the fundamental means for coupling electrical energy into a gas.

\subsection{Plasma Basics}

Plasma is usually described as being quasi-neutral because, on average, there are an equal number of positively and negatively charged species [113]. The presences of neutrals 
in plasma suggest that the volume of gas is partially ionized. This volume can be divided into two distinct regions namely:

\section{Bulk Plasma}

\section{Plasma Sheath}

The bulk region of plasma is what was earlier described as being a mix of ions, electron and neutral atoms. It is sustained by the numerous ionization and excitation events, provided the gas density and the applied voltage remain at the proper levels.

The plasma sheath is a region, less dense of charged particles, that forms between a surface, such as the container walls and the bulk plasma [113]. At such a surface, there are current densities due to both ions and electrons. However, the average electron thermal velocity, $\bar{v}_{c}=\sqrt{\frac{8 \mathrm{k} T_{e}}{m_{e}}}$, is about 3 orders of magnitude greater than the average ion thermal velocity, $\bar{v}_{i}=\sqrt{\frac{8 \mathrm{k} T_{i}}{m_{i}}}$, since an electron is about 100,000 lighter than an ion and its effective temperature $T_{e} \gg T_{i}[113]$. As a result, the electron current density is greater than the ion current density, $J_{e}>J_{i}$. This means any surface in contact with the plasma will initially begin to charge negatively with respect to the plasma and hence develop a less positive potential than the plasma itself. As a result, the ion flux increases and the electron flux decreases, until in the steady state, a space charge region is formed between the surface and the bulk plasma [113].

In figure 5.2(a), we see that initially the net charge density $\rho=q\left(n_{i}-n_{0}\right)$ is zero since $n_{e}=n_{i}$ and the plasma potential, $\phi$, and electric field $E_{x}$ is initially zero everywhere [86]. In 
figure $5.2(\mathrm{~b})$, we see the space charge region formation at the two walls of the container. As shown the ion current density ends up being slightly higher than the electron current density since the walls are grounded. The bulk plasma maintains itself as the most positive body in the discharge with a potential $V_{p}$.
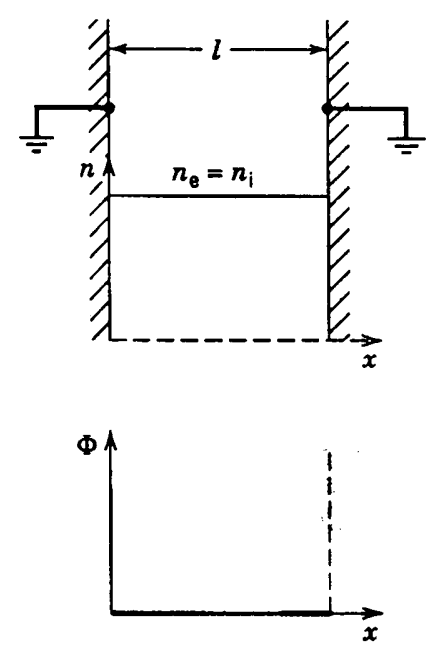

Figure 5.2(a) Initial state before sheath formation

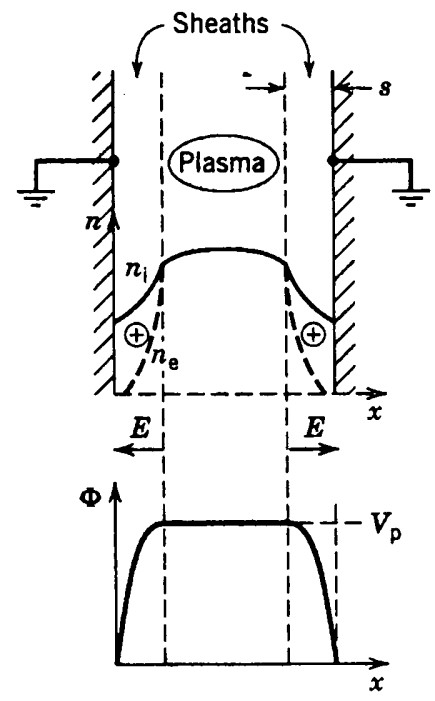

Figure 5.2(b) Plasma sheath formation 
If the surface had been floating, a potential $0<V_{f}<V_{p}$ would have developed on the surface causing the ion current density to less than what it was in the previous case. Finally, in the case of a negative electrode in a sputter system, the ion current becomes significantly higher than the electron current due the high negative potentials seen at such electrodes. The details of generating plasma for sputtering processes are explained in the next section.

\subsubsection{D.C. Plasma}

The DC sputtering configuration shown in figure 5.2.1 (a), provides us with the simplest model for generating plasma for film deposition purposes. In this model known as the parallel plate model or the planar diode model, a DC voltage is applied between the two electrodes as shown in figure 5.2.1 (a). The resulting voltage profile, after plasma formation, for this model is also shown in figure 5.2.1 (b).

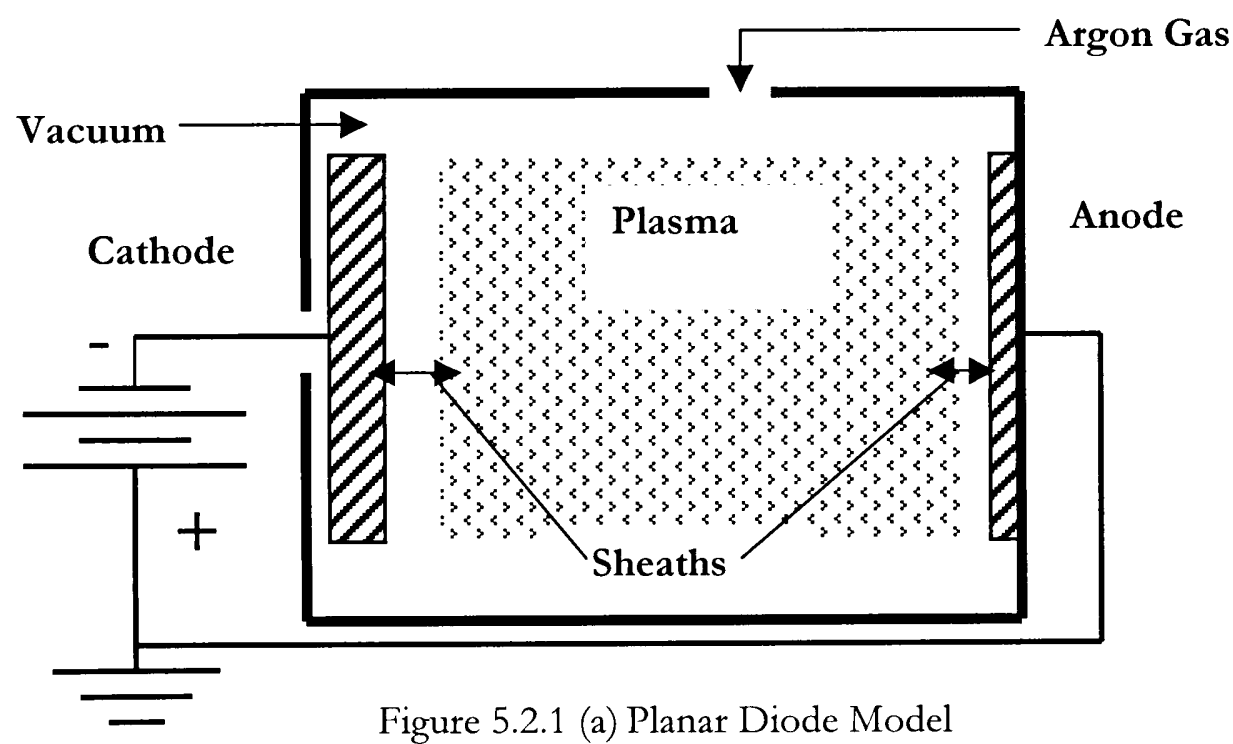




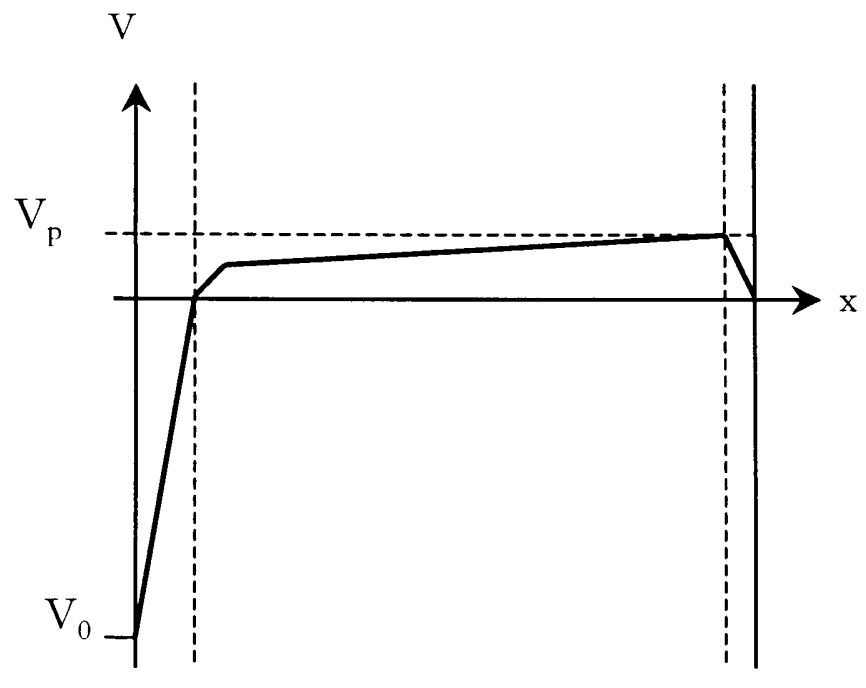

Figure 5.2.1 (b) Voltage profile for Parallel Plate Model

The material we wish to sputter is made into a "sputtering target" which becomes the cathode of the electrical circuit. This target is either metallic or insulating. If an insulating target is used, the material is usually a powder that has been sintered or hotpressed into a solid disk that is bonded to a copper cup to provide electrical contact. A large negative voltage $V_{o}$ is applied to the cathode while Ar gas is introduced into the vacuum chamber to some specific pressure. The substrate that we wish to coat is placed on an electrically grounded anode, usually a heater block made of stainless steel, some distance from the cathode.

Initially, electrons are accelerated by the electric field and in turn, collide with the Argon atoms inducing ionization as explained in the previous section. After the plasma is struck, the charged particles are accelerated by the electric fields in the sheaths. Although both ions and electrons are present in the sheaths, electrons are more heavily influenced at the anode while ions are predominant at the cathode and a current $I$ flows. Due to the large 
potential gradient at the cathode, Ar ions are accelerated with enough energy to sputter the target atoms away. They may also liberate secondary electrons, which are drawn into the plasma, sustaining the glow discharge $[88,89]$. The sputtered atoms fly of in random directions, but primarily toward the substrate, where they condense, nucleate, and form a thin film.

The voltage $V$ required to drive the current $I$ through the system is a function of the established gas pressure. The rate at which particles arrive at the substrate to form the film depends on the ion flux sputtering the target, and hence on the current. However, the ion energies required to sputter the material depends on the material being sputtered. Thus the yield of sputtered particles becomes dependent also on the voltage $V[113,90]$. Therefore the choice of sputtering pressure and the voltage-current relationship are important.

The glow discharge imposes a lower pressure limit. Electrons making ionizing collisions in the gas maintain the discharge. The number of ionizing collisions decreases with gas density, and hence gas pressure. The discharge current (for some constant voltage) will also decrease. Below approximately $30 \mathrm{mTorr}$, the current, and hence the ion flux at the target and thus the sputtering rate in a DC sputtering configuration, become quite small $[113,90]$.

Above a certain maximum pressure, a different problem arises. Sputtered material from the target may collide more frequently with gas atoms with a pressure increase. The result is more random deflection of the particles; sometimes back toward the target itself. This causes a decrease in the deposition rate. The reason lies in the fact that the mean free path of the sputtered particles decreases dramatically such that the deposition becomes less 
of a line-of-sight process and more of a diffusive process. Above $100 \mathrm{~m}$ Torr, the scattering process becomes more serious and hence the sputtering becomes ineffective [113].

Sputtering with DC plasma was the first method to be used and is still being used today. The method has its advantage in that the target is somewhat efficiently used and the operation of the system is simple. One of the disadvantages is that both the electrodes and the target must be good conductors. For insulating targets, the unipolar voltage characteristic at the cathode leads to charge accumulation on the target and can lead to arcing or even plasma extinction. Another major problem occurs when a reactive gas, such as oxygen, is needed for the sputtering films. This is the case when the film desired is a metallic oxide such as $\mathrm{MgO}, \mathrm{TiO}_{2}, \mathrm{ZrO}_{2}$ or $\mathrm{Al}_{2} \mathrm{O}_{3}[65,66,113,90]$. If oxygen is introduced into the DC plasma and the target is pure metal, the surface layers of the target rapidly become oxidized which decreases the ion current significantly, resulting in impractically low deposition rates.

\subsubsection{R.F. Plasma}

To be able to use insulating targets and metallic targets in a reactive process, an alternating voltage at a high frequency is used to generate the plasma. In a DC plasma, an insulating target at the cathode is bombarded by positive ions. The ions neutralize at the target surface through recombination with electrons that originate at the cathode. This leads to the surface layers of the target charging, like a capacitor, to some potential more positive than the cathode. The charging continues until, in the steady state, the surface potential of the target completely cancels out the potential at the cathode and the plasma is extinguished 
[113]. By using an AC voltage, the positive charge accumulated during the negative halfcycle can be neutralized by electron bombardment during the positive half-cycle [87]. The success of this depends on the frequency of the AC voltage used. The characteristics of the plasma also depend on the frequency used and hence two classifications exist:

$$
\begin{aligned}
& \text { 1. Low frequency }(<1 \mathrm{MHz}) \\
& \text { 2. High frequency }(>1 \mathrm{MHz} \text { but usually at } 13.56 \mathrm{MHz})
\end{aligned}
$$

At frequencies below $1 \mathrm{MHz}$, the $\mathrm{AC}$ plasma can be treated as a DC plasma that periodically reverses its polarity [91]. The plasma potential follows the oscillating electrode voltage as a half-wave rectifier with peak voltage of $V_{0}+V_{p}$ every half-cycle $[113,90]$. This is shown in figure 5.2.2 (a). At very low frequencies, the time taken to charge the surface of the insulating target is much less than the frequency of the alternating voltage and the result is a series of short discharges. At about $30 \mathrm{kHz}$, ions begin to respond slower to the electric field cycling and the electron current, during the positive half-cycles, begins to have greater influence. Because of the higher mobility of electrons, the cathode receives a much higher electron current during the positive half-cycle of the voltage than ion current on the negative half-cycle. In the steady state, the increasing effect of the electron current causes the cathode to develop a negative bias. This bias serves as potential to which the total ion flux on the target is proportional [113]. 
Figure 5.2.2 (a) Low Frequency Voltage vs. Time
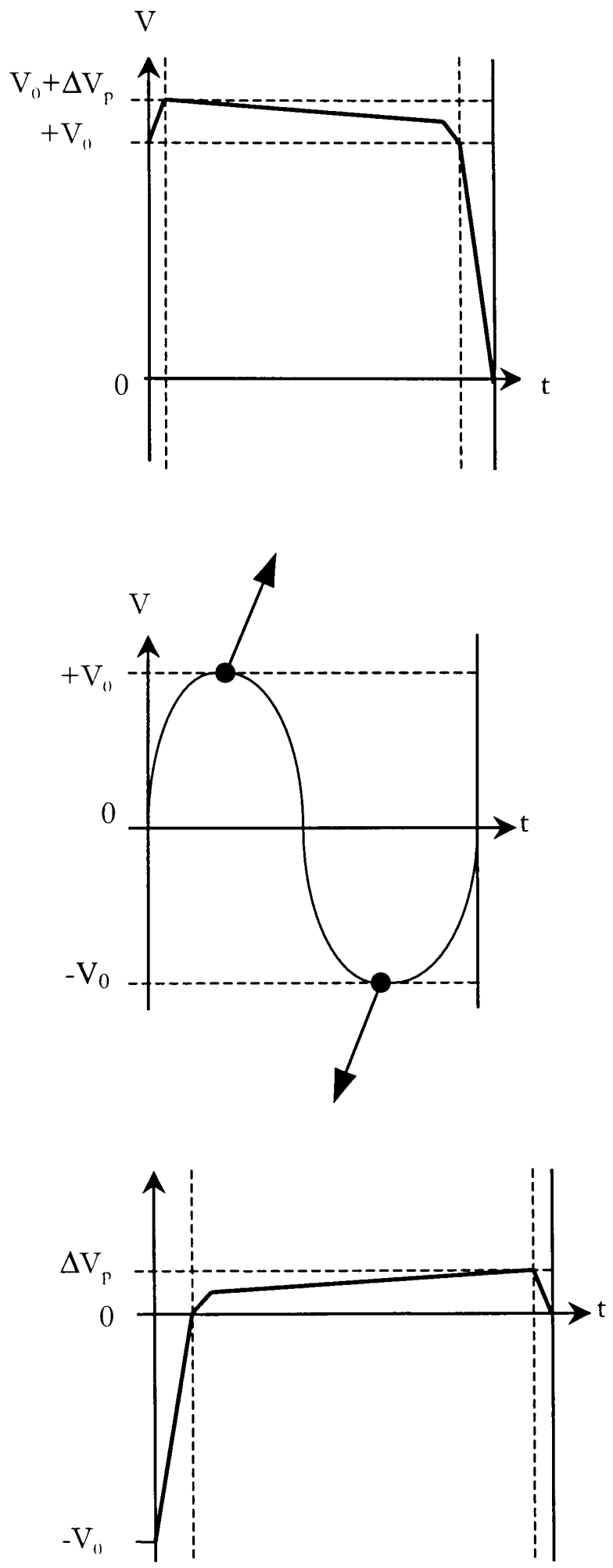
At frequencies above $1 \mathrm{MHz}$, plasma characteristics are notably different. First, the sheaths are no longer resistive. The oscillating electron current takes precedence over the ion current. This current is $90^{\circ}$ out of phase with the voltage suggesting that the sheaths are capacitive, the value of which is a function of the voltage at the cathode. Ions are now unable to respond to the rapidly changing electric field and hence are less influenced by the full voltage modulation [92]. Instead, the effect of the self-bias potential described in the previous section, is more pronounced. Now, positive half-cycles of the cathode voltage only exist for a very short time and the bias voltage approaches a value nearly half of the total RF peak-to-peak voltage. This leads to the target experiencing ion bombardment almost continuously.

There is still a significant electron current during the positive half-cycle, but it is exhibited at a lower potential. However, the effect of the oscillating field serves to accelerate the electron impact ionization process also. This can be explained by noting that if an electron undergoes an elastic collision and the recoil occurs exactly when the electric field changes direction, then the velocity and hence kinetic energy of the electron will continue to increase. In this way, electrons can obtain ionizing energies with a relatively weak electric field. For this reason, RF plasmas are able to sustain the glow discharge at lower pressures than DC plasmas. They can operate at pressures as low as $1 \mathrm{~m}$ Torr, enabling sputtered particles to be deposited with more directionality with fewer collisions [93].

At high frequencies, capacitors are required to couple power to the target to ensure impedance matching for maximum power transfer. The absence of charge build-up on the target allows high deposition rates to be maintained [89]. The fact that ions are not able to respond to quickly changing electric field leads to a plasma voltage maintaining a positive 
polarity during the negative half-cycle of the RF voltage [87]. The result is that the plasma voltage varies in a roughly sinusoidal form, proportional with the sheath capacitance. This is illustrated in figure 5.2.2 (b).

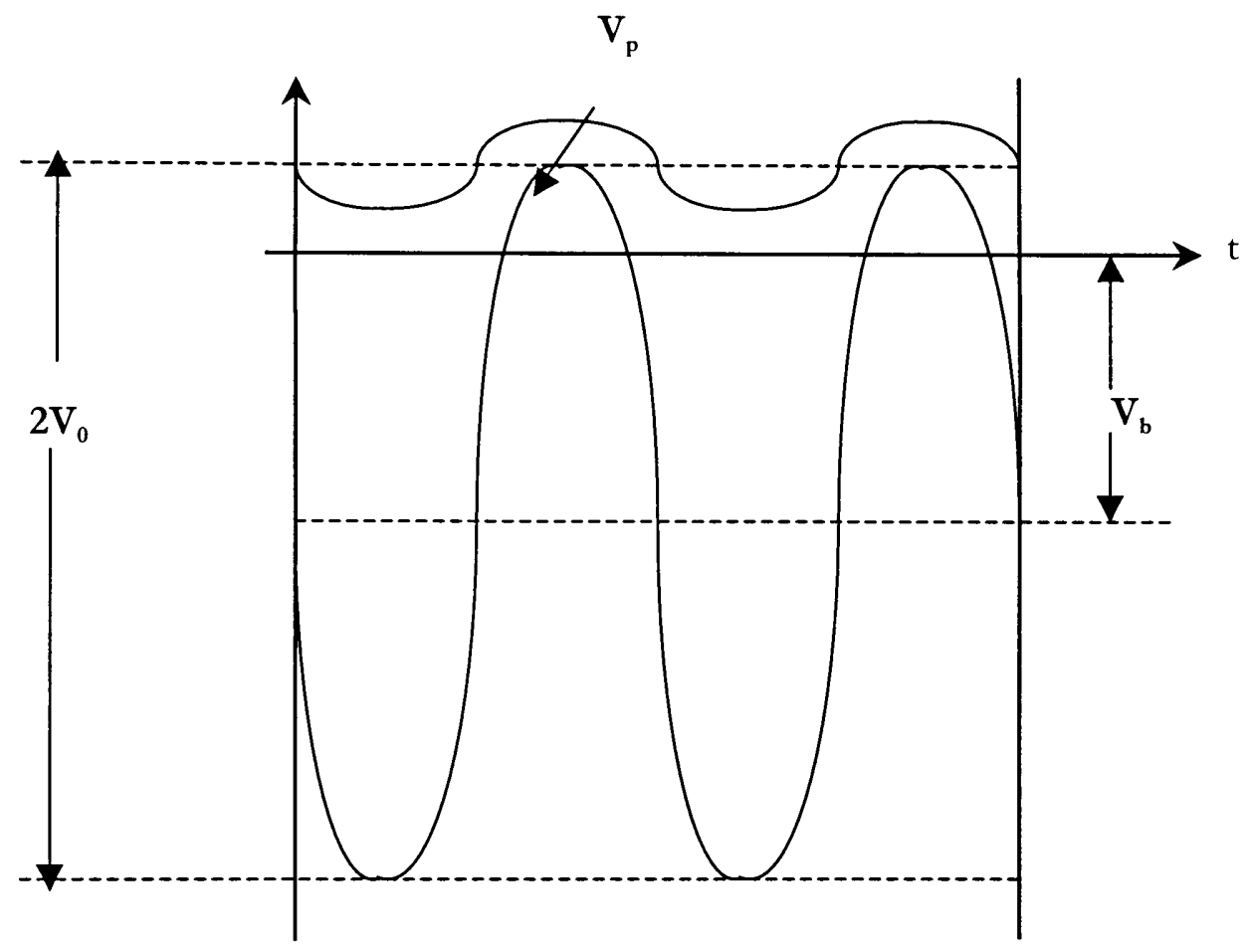

Figure 5.2.2 (b) High Frequency Voltages vs. Time

Typical RF frequencies used are between 5 to $30 \mathrm{MHz}$ but the Federal Communication Commission (FCC) has set $13.56 \mathrm{MHz}$ for sputtering operations. 


\subsubsection{Pulsed D.C. Plasma}

The case of using square wave excitation at frequencies of $30-120 \mathrm{kHz}$ to generate plasma for sputtering purposes has gained interest in recent years [81-83,94-97]. The behavior of such pulsed D.C. plasma is similar to regular D.C. plasma except that insulating targets able to be used. A typical pulsed D.C waveform is shown in figure 5.2.3.

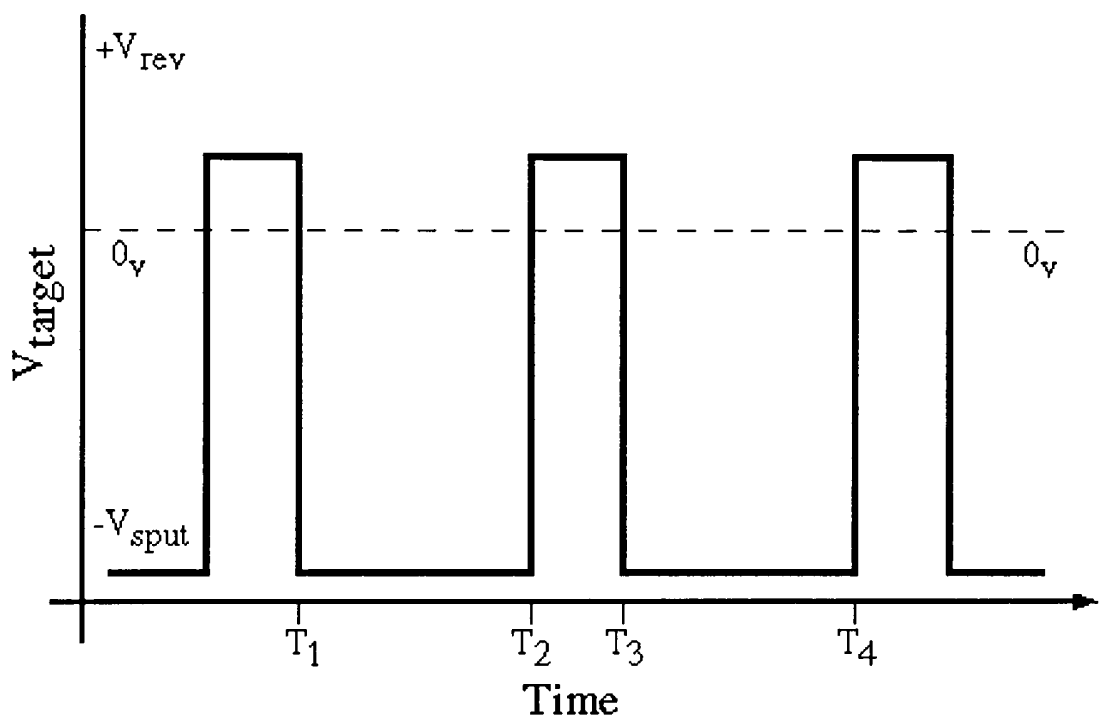

Figure 5.2.3 Pulsed D.C. waveform

During the negative pulse, sputtering of the target takes place. The pulse duration is kept long enough just to prevent too much of a charge build up on the target. The positive pulse that follows is generally shorter such that the overall deposition rate is unaffected since deposition only occurs during the negative pulse. Therefore, the positive pulse is kept short enough just to allow enough of an electron flux to discharge the surface layers on the target. Experiments have shown that, depending on the target material, the time required to 
accomplish this can be as short as one microsecond to as long as 50 microseconds. If the duty cycle of the waveform is not optimized, arcing can still occur through incomplete discharging of layers. For this reason, duty cycles of $20-40 \%$ are typically used $[81,98]$.

The overall effect of using pulsed D.C. power is that deposition rates are higher than those obtained with R.F. power, in the case of insulating targets or reactive deposition. This leads to films that have a greater packing density with good film morphology. Typically, methods of increasing the deposition rate with R.F. sources include increasing the sputtering power. This has adverse effects in that there is greater electron bombardment on the nucleating film and hence disturbs the growth process. The method of pulsed D.C was used in this project and more detail on the process in described in the next chapter.

\subsubsection{Magnetrons}

To further enhance the deposition process, Magnetron Sputtering is widely used for thin film deposition [99-104]. There are different variations of magnetron operation, such as cylindrical, balanced and unbalanced [104-107]. The planar disk balanced magnetron will be discussed in this section, as it is the only type used in this work. The basic planar magnetron is shown in figure 5.2.4 (a).

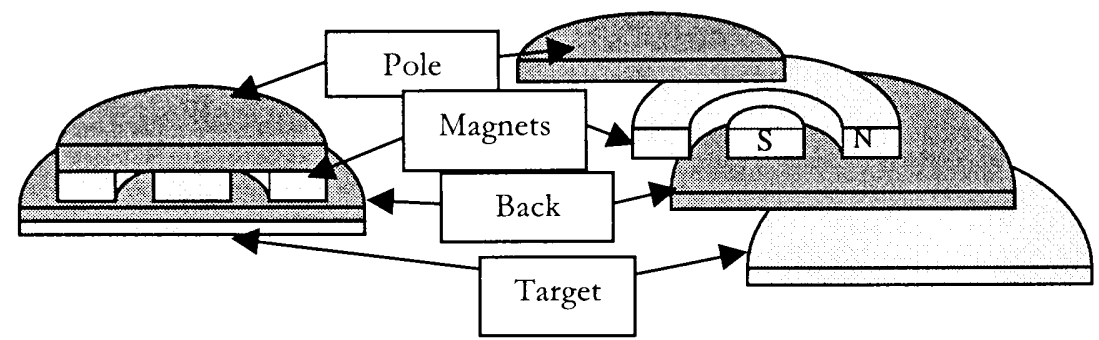

Figure 5.2.4(a) Planar Disk Magnetron Source 
The magnetron differs from standard diode configurations explained in the previous section with magnets ( 50 to $500 \mathrm{G}$ ) being placed behind the target as shown in the figure. This produces a magnetic field that is perpendicular to the electric field lines set up by the cathode, in a circular region in front of the target. The magnetic field lines originate from the center of the target and bend semi-circularly to complete the magnetic circuit at the ends of the target [91]. By the Lorentz force law [108],

$$
F=q(E+\mathbf{v} \times \mathbf{B})
$$

the component of the magnetic field parallel to the target causes a force on electrons that tends to pull them toward the target. This leads to electrons being trapped near the target in a circular orbit. The trapping mechanism causes more electrons to be involved in ionization processes than being lost to the walls of the chamber and the anode. The magnetron operation is shown in figure 5.2.4(b).

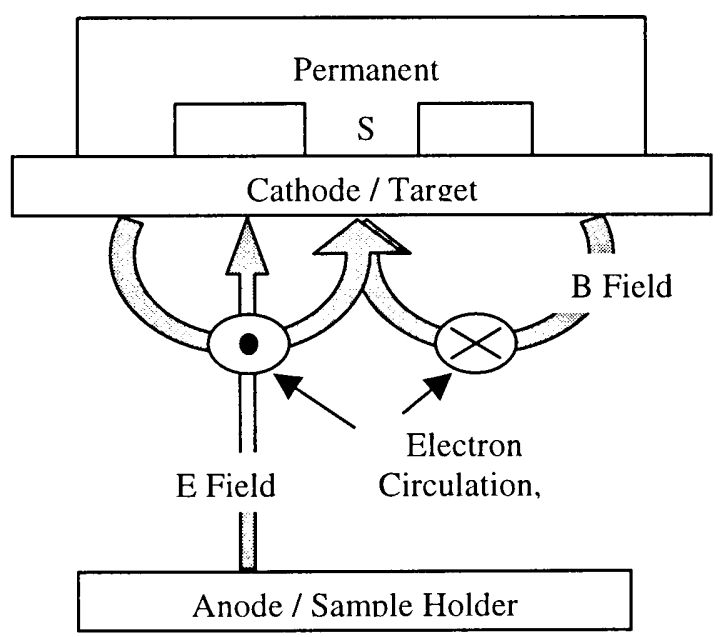

Figure 5.2.4(b) Magnetron Operation 
The actual motion of the electrons in the circular path at the target is cyclodial. Those that don't aid the ionization process reach the target surface at the end of every revolution of the cycloid. This action enhances the erosion of the target and helps sustain high, stable deposition rate [113].

Magnetron sputtering is the most widely used sputtering method in industry. The ability to sustain glow discharges at low pressures and the ability to obtain higher deposition rates than with planar diode configuration makes magnetrons attractive. It is important to note that since the average velocity of ions is much less than that of the electrons, they are virtually unaffected by the magnetic field. They are, however, affected by the electron motion through electrostatic attraction that can lead to charge exchange and their neutralization [92].

\subsection{Sputter Deposition of YSZ Thin Films}

Sputter deposition is widely used in industry and research to deposit a variety of materials. Metals, alloys, inorganic compounds, and ceramic thin films may be sputtered [109]. Common metals that are sputtered include copper and aluminum for contact metallization. Compounds such as silicon dioxide, magnesium oxide, zirconium oxide, silicon nitride and titanium oxide are also common materials that are sputtered, to name a few. Films may be sputtered from a target composed of the exactly the material desired. This form of deposition is referred to as direct sputtering. Alternately, multi-component films may be deposited by sputtering individual targets of each compound. This method is called co-sputtering and is generally more complicated to implement. Ensuring that each 
target is sputtered at the proper rates to obtain the required composition is hard to control. Small deviations in the proper rates of sputtering can cause poor homogeneity in film composition. Another method of depositing compound films is to react a gas, such as oxygen or nitrogen, with the sputtered material to obtain the desired material. This is known as reactive deposition.

\subsubsection{Direct Sputtering}

Direct sputtering has its advantages and disadvantages. Using a single target as the advantage of simple operation. The disadvantage appears when compound targets are used with elements that have different sputtering rates. The result is that preferential sputtering of one element over another may occur. For instance, when sputtering YSZ targets at high power levels, oxygen may disassociate yielding films that are oxygen deficient. As a result careful choice of power levels is important. To counteract the disassociation problem, a small flow of oxygen may me needed during the deposition. Another consideration is how well a material will sputter. Metallic materials are easily sputtered with R.F., D.C. or pulsed D.C. power, and have high sputter yields. Insulating materials such as YSZ have to be sputtered using R.F. or pulsed D.C. power and generally have low sputter yields. An interesting thing to note is that depending on how these targets are made decides whether pulsed D.C. power may be used. If the target is completely insulating, then pulsed D.C. power may not be suitable. In the case of YSZ, the method of manufacture yields targets that are either not completely oxidized (hot-pressed) or completely oxidized (vacuum sintering). In our own experiments, the hot-pressed targets worked well using pulsed D.C. 
power. Deposition rates were high and stable. When the sintered targets were used with pulsed D.C., deposition rates fell dramatically. This is explained by the fact that the sintered targets, being fully oxidized, are susceptible to charge build up in a much shorter time. The low frequency of the positive cycle is not fast enough to completely discharge the target. Thus the sputtering rate is kept at a bare minimum.

\subsubsection{Reactive Sputtering}

Reactive deposition is attractive since high deposition rates can be realized when depositing insulating compounds. In the case of YSZ, a pure $\mathrm{Zr}$ target on which pieces of $\mathrm{Y}$ metal are placed can be sputtered in an $\mathrm{Ar} / \mathrm{O}_{2}$ atmosphere to produce $\mathrm{YSZ}$ films [74]. The advantage here is that not only can the yttria content be controlled by how many pieces $\mathrm{Y}$ are used but also the growth rate of YSZ can be very high. This is possible since by keeping the target in "metallic" mode high sputtering rate is maintained. Ideally, the reaction to form the oxide takes place within the plasma and at the substrate where the compound forms. The disadvantage is that the partial pressure of the reactive gas (in this case $\mathrm{O}_{2}$ ) must be very carefully controlled in order to maintain the "metallic" condition at the target. If this is not done, the target enters the "poison" mode. In this mode, very thin oxide layers begin to form on the target surface that are enough to reduce the deposition rate and cause instabilities in the plasma. The other problem is that the substrate may react with the reactive gas. In the case of $\mathrm{Si}, \mathrm{SiO}_{2}$ forms on its surface causing disorientation in the growing YSZ film. 
Many different methods of partial pressure control exist. The use of mass spectrometers to sense the oxygen has been employed as a feedback controller for oxygen flow $[65,83]$. As the target approaches the "poison" mode, the partial pressure of oxygen increases. Oxygen flow is increased or decreased as needed to maintain a fixed partial pressure at which this is prevented. Another method of preventing target poisoning is to monitor the bias voltage. As the target becomes poisoned, the bias voltage decreases in magnitude since the current to the target is reduced. By monitoring this voltage, oxygen flow can be controlled to maintain a voltage that keeps the target in the "metallic" mode.

\subsubsection{Gas Delivery}

Argon may be delivered to the chamber from anywhere. Typically, it is let in through a stainless steel tube at the base or sides of the chamber (see figure 5.2.1(a)). Oxygen may be introduced in a similar manner or be let in close to the target. At first this may seem to nullify the cause of keeping the target non-oxidized. However, if very small flow rates are used in combination with partial pressure control then no problem is posed. The advantage of this method is that the chance of the substrate reacting with oxygen is kept to a minimum. For the case of substrates that do not react, another option is to flow oxygen through a gas ring tube close to the substrate. This lowers the chance of the target oxidizing and oxygen control may not even be needed. 


\subsubsection{Substrate Positioning}

The sample or substrate is usually mounted on flat stage oriented on the axis of the sputtering target. The sample stage is usually a heater block that serves to heat the substrate to a desired deposition temperature. The substrate is affixed to the heater such that there is proper thermal contact, typically by using a colloidal silver paste or through the use of clamps. Alternatively, the sample can be positioned with different orientations relative to the target. This is referred to as being off-axis with the target. The on-axis and off-axis geometries are shown in figure 5.3.4(a) and 5.3.4(b).

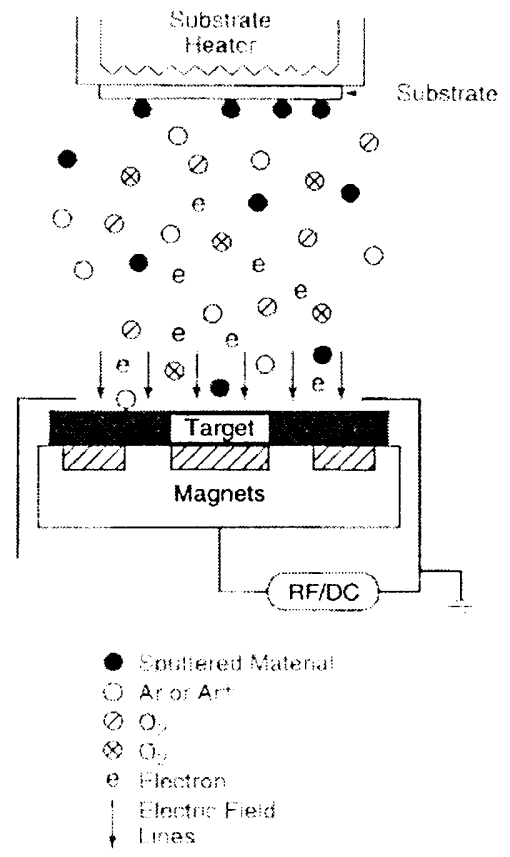

Figure 5.3.4(a) On-axis Configuration 


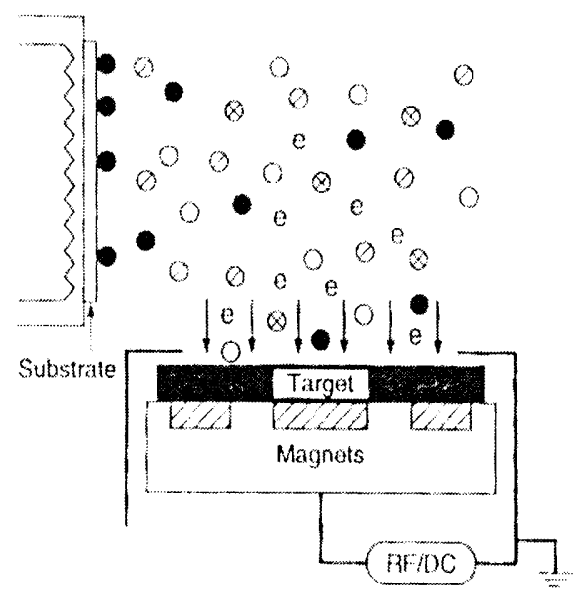

- Soultered Material

() Aror $\mathrm{As}^{+}$

() $\mathrm{O}_{2}$

(i) $\mathrm{O}_{3}$

e Electron

Electris Fred

- Lines

Figure 5.3.4(b) Off-axis Configuration

In the on-axis configuration, the sputtered flux is parallel to the electric field lines and perpendicular to the substrate. A portion of ions and electrons from the plasma discharge do hit the substrate surface and can have adverse effects on the growing film. In the off-axis geometry shown, the substrate is oriented $90^{\circ}$ to the particle flux. This has the effect of reducing the kinetic energies of the ions and electron such that they do not influence film growth as much. The disadvantage of off-axis sputtering is that the deposition rates are extremely low compared to on-axis. Substrate rotation is generally needed to ensure uniform film growth but the nucleation process of the thin film may be haphazard or even change completely from what is expected. 


\subsection{Sputter System Description}

The sputtering system used in this work is a custom built unit designed and manufactured by Cooke Vacuum Products (Norwalk, Connecticut U.S.A). A photograph of the system appears in figure 6.1.

A full description of the system and its equipment configurations appear in this chapter. Details of the thin film deposition experiments and some techniques of film characterization are also described. 


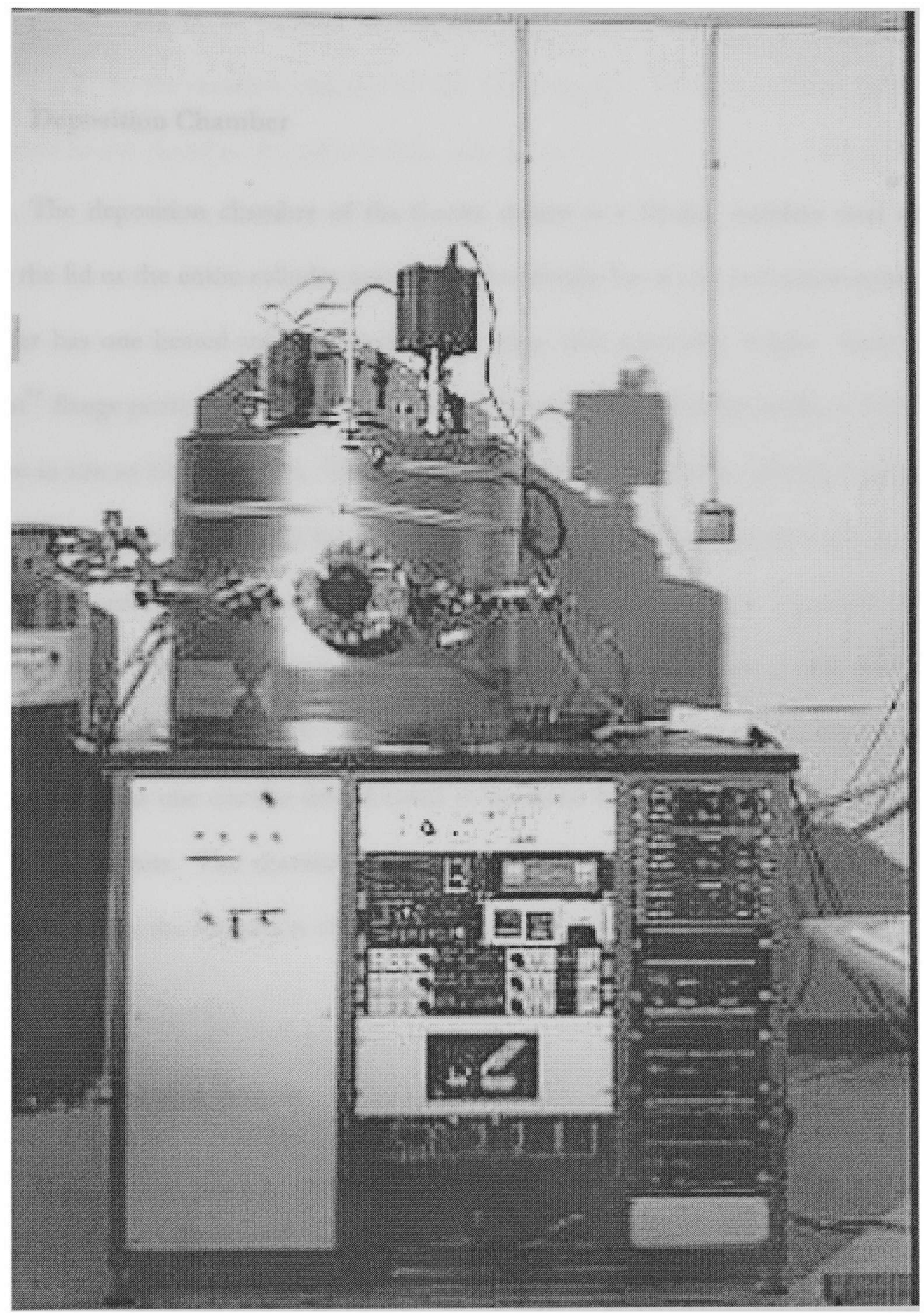

Figure 6.1 CVP Sputter System 


\subsubsection{Deposition Chamber}

The deposition chamber of the Cooke system is a $43-\mathrm{dm}^{3}$ stainless steel cylinder where the lid or the entire cylinder may be lifted vertically for access and maintenance. The chamber has one heated stainless steal sample stage with adjustable height. There are six Conflat ${ }^{\mathrm{TM}}$ flange ports spaced around the circumference of the chamber walls, of which only two are in use as viewing ports. The spare ports provide for flexible off-axis configuration of multiple sputtering guns and for mounting of in-situ analysis equipment such as residual gas analyzers and film thickness monitors. There is another flange at the top of the chamber for use with an on-axis sputtering gun. The breakable vacuum seals at the base of the chamber and on the lid are made with Viton o-rings while all the flanges use copper gaskets. The chamber has one circular door located at the front that is used for access and also for viewing the process. The chamber walls are externally heated using band heaters. This is done to minimize the formation of water vapor on the inside of the walls.

\subsubsection{High Vacuum System}

The vacuum system uses two mechanical pumps and one turbo pump, with appropriate valves. The two mechanical pumps serve two purposes. The larger of the two is used to evacuate the chamber from atmosphere $(760$ Torr) to an intermediate pressure of $50 \mathrm{~m}$ Torr. At this pressure, pumping is crossed over to the turbo pump by way of a swing gate valve. The second mechanical pump is smaller and uses Fomblin ${ }^{\mathrm{TM}}$, a special fluid that 
prevents contamination of vacuum system with carbon seen when regular oil is used. This pump serves at the foreline exhaust to the turbo pump. Both mechanical pumps are connected to the chamber through stainless steel piping with in-line zeolite traps. The traps help to block the potential back-streaming of the mechanical pump fluid. The turbo pump can only operate at or below $50 \mathrm{~m}$ Torr and will fail if operated at higher pressures. This pump is responsible for pumping the system down to pressures $<10^{-6}$ Torr. The pump has a quality factor of 10,000 meaning that it, ideally there are four orders of magnitude in pressure difference between the high vacuum and the low vacuum sides. Hence the need to use a mechanical pump on the low vacuum side to "back" the turbo pump becomes evident. Since most mechanical pumps provide ultimate pressures of $\sim 10^{-3}$ Torr this suggests that the ultimate pressure that the turbo pump can achieve is $\sim 10^{-7}$ Torr. The turbo pump uses magnetic bearings, thus requiring absolutely no oil for its operation. This eliminates the possibility of chamber contamination.

The foreline, roughing, trap and vent valves are all binary. There is a throttle valve whose opening can be adjusted to vary the pumping speed of the turbo pump.

\subsubsection{Gas Flow Control}

The flow of gases into the sputtering system is an important factor in any sputtering system. The sputtering gas, argon, is flowed into the chamber using a mass flow controller (MFC). The MFC is able to flow 1 to 100 standard cubic centimeters (sccm) of argon with a resolution of $0.1 \mathrm{sccm}$. The flow of argon is used to establish and sustain the sputtering pressure in the chamber. After a desired flow is set, the turbo pump and the position of the 
throttle valve maintain the pressure. The typical sputtering pressure is in the range of $10^{-3}$ to $10^{-2}$ Torr. As an example, a flow of $20 \mathrm{sccm}$ of argon into the chamber establishes a pressure of $2.8 \times 10^{-2}$ Torr with the throttle valves closed. The system has a second MFC for controlling the flow of oxygen. This controller has a range of 0.1 to $20 \mathrm{sccm}$ with a resolution of $0.01 \mathrm{sccm}$. The flow may be controlled remotely through an on-board RS-232 port.

The argon is let in through a stainless steel tube at the base of the chamber. Oxygen can be delivered through three different paths. It can be let in through a stainless steel tube also at the base of the chamber, through a gas ring tube close to the substrate heater stage, or directly onto the target face. There are isolation valves between the MFCs and the chamber. This prevents leakage from the MFCs, which are not positive closure devices. They can leak up to $1 \%$ of their maximum flow, even when they are turned off, preventing high base vacuum level from being achieved.

\subsubsection{Pressure Gauges}

The system has three pressure gauges. Two of them are thermocouple types and the other is an ion gauge. One of the thermocouple gauges monitors the pressure in the foreline. The other thermocouple gauge and the ion gauge monitor the chamber pressure. The thermocouple gauges have an operating range of $7 \times 10^{2}$ to $1 \times 10^{-3}$ Torr and the ion gauge operates in a range from $5 \times 10^{-4}$ to $<10^{-8}$ Torr. 


\subsubsection{Substrate Stage}

The substrate stage is the top of a 10.16-cm diameter cylinder. A resistive heater element made of Kanthal is set inside a Zirconia ceramic plate and a thermocouple is inside the cylinder, which is bolted to a $13.3-\mathrm{cm}$ Conflat $^{\mathrm{TM}}$ flange. The flange is welded to a $2.54-$ $\mathrm{cm}$ diameter stainless steel pole that runs through the center of the base of the chamber. The height of the stage can be adjusted from 7 to $31 \mathrm{~cm}$ with respect to the base of the chamber. The stage may be heated to a maximum temperature of is $900^{\circ} \mathrm{C}$. There is, however, a $120^{\circ} \mathrm{C}$ temperature gradient observed between the inside of the heater cylinder and the actual substrate temperature. Substrates are either simply placed on the stage or affixed to it using silver paste to improve thermal contact. In this project, all experiments were carried out with glued down substrates. The stage itself can also be rotated to provide uniform film growth during off-axis sputtering. The rotational rate can be varied between 0 to $1 \mathrm{rpm}$.

\subsubsection{Sputtering Guns and Targets}

The sputtering system has seven identical planar magnetron sputtering guns of which only two are installed. One is mounted through a port in the sidewall of the chamber providing the option for $90^{\circ}$ off-axis depositions. The other is mounted through a central port in the lid of the chamber and is oriented directly above the sample stage for on-axis depositions. This is illustrated in figure 6.1.6. The guns can accommodate $5.08 \mathrm{~cm}$ (2-inch) disk targets with $0.3175 \mathrm{~cm}(1 / 8$-inch) to $0.635-\mathrm{cm}(1 / 4$-inch) thickness. Each gun is 
mounted on a shaft to allow adjustment of the height/distance from the substrate. The range of motion depends on the position of the sample stage.

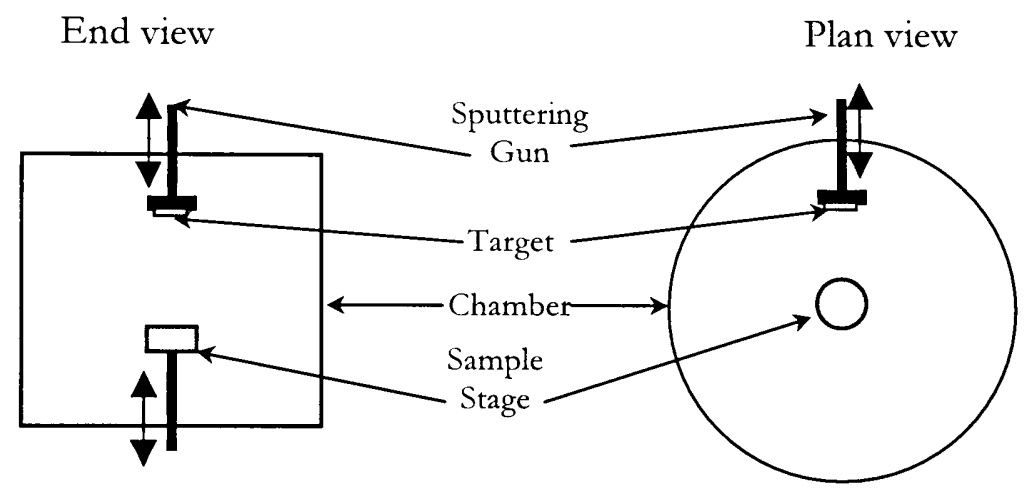

Figure 6.1.6 Sputter System Geometry

Two $99.9 \%$ pure 8 mole \% Yttria-Stabilized Zirconia $\left(\mathrm{Y}_{2} \mathrm{O}_{3}\right)_{0.118}\left(\mathrm{ZrO}_{2}\right)_{1-x}$ targets were used. Both were $5.08 \mathrm{~cm}$ in diameter bonded to a $0.636-\mathrm{cm}$ thick copper cup. The only difference between them is their method of manufacture. One target which we will call target $A$, was manufactured using the vacuum hot-pressed method and the other, target $B$, by the sintering method. The details of the processing will not be explained here. However, one important thing to mention is that the hot-pressed target loses oxygen during processing while the sintered targets remains fully oxidized. This has the effect of lower conductivity in the sintered target as compared to the hot-pressed target. YSZ is known to have a large oxygen diffusion coefficient of $2 \times 10^{-9} \mathrm{~cm}^{2} / \mathrm{s}[68,69]$. This causes the oxygen deficient target to respond differently during sputtering. First, a nominal flow of oxygen is required, during deposition to ensure proper oxygen stoichiometery in the grown films. Second, the target can form thin, fully oxygenated layers that alter the bias voltage at the cathode and can cause instability in the deposition rate. However, because of its greater electrical 
conductivity, pulsed D.C. power may be used to counter the problem. The sintered target's conductivity is too low for pulsed D.C. power and R.F. power then has to be used. The advantage of this target is that little or no oxygen is required during deposition.

\subsubsection{Plasma Drive Units}

The sputter system has seven plasma drive power supplies. Three are R.F. sources (RFX-600, Advanced Energy Corp) that supply up to $600 \mathrm{~W}$ of power and three are D.C. sources that supply a maximum of $500 \mathrm{~W}$ (MDX-500, Advanced Energy Corp). The seventh is a Pulsed D.C. (PDC) unit that has a maximum power of $5 \mathrm{~kW}$ (RPG-50, ENI Corp). The multiple plasma drive units make provision for multi-target depositions if needed. The three R.F. supplies RF1, RF2 and RF3 provide a variable amplitude sinusoid at 13.865 MHz. D.C. units DC1, DC2 and DC3 produce negatively polarized D.C power. The pulsed D.C. supply provides a variable amplitude, asymmetric bipolar square wave at frequencies of $30-200 \mathrm{kHz}$ with an adjustable duty cycle. The R.F. units are connected to the sputter guns via fully automatic impedance matching units. The matching units can also be manually controlled if desired. For our experiments only the R.F. and the PDC unit were used.

\subsection{Experimental Procedure}

To find the ideal parameters for deposition of a desired material, many experiments must be performed with equipment parameters being varied over a wide range. It is not 
possible to predict the change of a given parameter on the process. Therefore empirical data is taken on measurable effects such as changes in deposition rate and film structure as process parameters are changed. In our experiments the goal was to find the best thickness of YSZ film, that when grown on Si, provides a good buffer layer for YBCO films. Process parameters that were varied systematically over a number of experiments include:

1. Ar: $\mathrm{O}_{2}$ flow ratio.

2. Total sputtering pressure.

3. Plasma source power level (RF and PDC).

4. Target to substrate distance.

5. Deposition time.

6. Substrate temperature.

\subsubsection{Substrate Preparation}

The substrates used in these experiments were all (100) oriented, p-type 53-56 $\Omega$-cm, silicon, cut into $15 \mathrm{~mm}^{2}$ square pieces from a $100 \mathrm{~mm}$ diameter wafer. The wafers are shipped with less than $10 \mathrm{~nm}$ of native oxide to keep the silicon surface as clean as possible. These pieces were first degreased in acetone, dipped in ethyl alcohol and finally rinsed in deionized (DI) water. They were then stored in square plastic boxes for use as needed.

In preparing for a deposition, one or two Si pieces are selected and cleaned in a solution of 10:1:1 $\mathrm{H}_{2} \mathrm{O}: \mathrm{HCL}: \mathrm{H}_{2} \mathrm{O}_{2}$ to remove any organic and heavy metal contaminants that may be present. Afterwards, the substrates were rinsed in DI water and their surface oxide 
removed by dipping in a 10:1 solution of diluted hydroflouric acid for 10 seconds. Finally they were blown with dry $\mathrm{N}_{2}$ prior to loading into the deposition chamber.

\subsubsection{Sputtering Sequence}

Each deposition was carried out following a standardized sequence of steps, which is listed below. In a few cases minor deviations were made to see if they would have any significant effect.

Sputter Deposition Cycle:

1. The high-vacuum valve is closed and the chamber is vented to atmospheric pressure when the sample stage is below $100^{\circ} \mathrm{C}$. The previous set of sample(s) is removed with a thin blade and the sample stage is cleaned with sand paper.

2. The new set of samples is placed on the sample stage with silver paste on the back.

3. The chamber is sealed and rough pumped with the mechanical pump.

4. The gas line isolation valves are opened to release an pump out any trapped gas.

5. The sputtering guns are raised or lowered to their chosen distance.

6. When the pressure in the chamber reaches crossover pressure $\left(5 \times 10^{-2}\right.$ Torr $)$ the roughing valve is closed and the foreline valve is opened. The throttle is closed and the high-vacuum valve is opened. After a few moments the throttle is opened and the ionization pressure gauge is turned on. 
7. The chamber is then pumped down to a predetermined base pressure, normally between $5-8 \times 10^{-6}$ Torr.

8. Sometimes, during the pump down, an RGA mass spectrometer is used to check for leaks or contamination within the chamber volume. This model only operates at or below $5 \times 10^{-5}$ Torr.

9. On reaching the base pressure, the sample stage heater is set to the desired temperature.

10. The ion gauge is deactivated and the throttle valve is closed.

11. The desired pressure of argon is established by setting an appropriate flow rate.

12. The plasma is struck using the chosen power supply (RF or PDC).

13. The target is covered with a metal shutter and sputtered for a predetermined time in order to uncover fresh layers for deposition. This is sometimes done with oxygen flowing across the target.

14. Upon the substrate reaching the desired temperature, the target is uncovered and the deposition begins.

15. The deposition ends.

16. When the deposition has been completed, the gas flows are stopped, the gas isolation valves are closed and the throttle is reopened.

17. The chamber remains under turbo-pumping until the sample stage has cooled sufficiently for step 1 to take place. 


\subsubsection{Deposition Parameters}

The following sub-sections detail the deposition parameters stated at the beginning of section 6.2. From the literature, we were able to establish a suitable starting point in parameter space, for our experiments. However, for the purpose of optimizing for our needs, we investigated the effect each had on the overall process.

\subsubsection{Argon Flow/Total Pressure}

For all depositions Ar was used as the sputter gas. The flow rate of argon accounts for the majority of the total pressure in the chamber. Oxygen when used, accounts for $25 \%$ or less of the total pressure. As R.F. and P.D.C. power was being used, a reasonably low operating pressure was chosen nominally to be $10 \mathrm{mTorr}$. This corresponded to a having an argon flow of approximately $20 \mathrm{sccm}$. Experiments were carried out in a range of $6-320$ mTorr. Below 6 mTorr, it was hard to sustain a stable plasma discharge. Going up to 320 mTorr was done to observe the effects of high pressure when using R.F. or P.D.C. power. The total pressure, in most cases, was a result of an $\mathrm{Ar} / \mathrm{O}_{2}$ gas mixture with ratios of 4:1 to 40:1.

Because of the small amount of oxygen present, the total pressure has its greatest influence on the mean free path of oxygen atoms. If the pressure is low and the targetsubstrate distance is greater than the mean free path of oxygen, then oxygen atoms have a greater chance of reaching the substrate and cause back-sputtering of the growing film. This is not the case if the target-substrate distance is equal to or less than that mean free path. 
Thus the target-substrate distance also plays an important role which is also influenced by the total sputtering pressure. This is further elucidated by noting that even the material being sputtered has its chances of reaching the substrate undisturbed, heavily influenced by the total pressure. If the pressure is too high, a large amount of scattering of the sputter flux occurs since the mean free path is small, and this leads to more random deposition. The argon pressure also influences the sputtering rate of the target.

\subsubsection{Target-Substrate Separation}

The target's distance from the substrate may be varied over a wide range of $<1 \mathrm{~cm}$ to $23 \mathrm{~cm}$ relative to the vertical set point of the sample stage. From previous work we determined that using the full distance was futile because the deposition rates became just to small to be useful [66]. One experiment, however, was done where high power and low total pressure were used to deposit a $\mathrm{ZrO}_{2}$ film from that distance. Analysis showed that good films had been grown, however they were also very thin. There are some advantages to the so-called "long-throw" method of sputtering, but the proper configuration is quite complex [110]. However, in our experiments we confined our range of separation to be between 1 and $7 \mathrm{~cm}$. The deposition rates were measured for each separation used at a corresponding power level. 


\subsubsection{Power Levels}

The fact that YSZ is an insulator completely rules out using D.C power as stated in previous sections. Several depositions were made using the R.F. and P.D.C. supplies used at levels of 50 to $100 \mathrm{~W}$. This corresponds to power densities of 2.46 to $4.93 \mathrm{~W} / \mathrm{cm}^{2}$ appearing on the target face.

For the R.F. supplies, auto-tuning matching networks ensured good impedance match for maximum power transfer. The network's controller allows the operator to manually control tuning. It also displays the operating bias voltage. The power supplies display the operating power and also the magnitude of reflected power. Interconnection is by means of shielded RG-58U coaxial cables. The negative terminal is connected to sputtering gun (cathode) via the matching network and the ground return is ultimately connected to system ground, which includes the steel system chamber. Care is taken to ensure signal shorting of the sputtering gun does not occur. For each experiment, the operating power, reflected power and bias voltage were recorded. The bias voltage is an important parameter as it is affected by sputtering pressure and oxygen partial pressure.

The P.D.C. unit, being a low frequency source, does not require matching components and therefore is connected directly to the gun. The unit has a multi-function display that tells the operating power, voltage and current. The system also includes arc suppression circuitry that can extinguish micro-arcs from dielectric breakdown that occur during reactive deposition or when insulating targets are used. The frequency, duty cycle, and time period for arc suppression can be adjusted. For our experiments, frequencies of 72 $-145 \mathrm{kHz}$ were used with a duty cycle of $27-52 \%$. 


\subsubsection{Deposition time}

Deposition time generally only influences the final thickness of the film grown. However, another effect that might appear is changes in the substrate condition during deposition. Especially, in a reactive process using oxygen, Si substrates may become oxidized during deposition leading to poor growth surface for any depositing film. It was noted in our experiments that the faster the YSZ films were grown, the less effect the $\mathrm{Si}$ oxidation had. Therefore a key goal was to always maximize the deposition rate when possible with minimum deposition time. This involved carefully balancing the power levels, sputtering pressure and the overall deposition time.

The critical thickness of YSZ on $\mathrm{Si}$ that ensures minimum cracking from stress/strain was determined to be between 50 to $100 \mathrm{~nm}$. This provided a favorable growth surface for the YBCO films that were subsequently deposited on the YSZ/Si structure. In order to determine the proper deposition time that achieves this, considering total pressure levels, power levels and target-substrate distance, experiments were run for a length of $5 \mathrm{~min}$ to as long as $2 \mathrm{hrs}$.

\subsubsection{Substrate Temperature}

The substrate temperature is one of the easier deposition parameters to deal with. The only effect it has on the deposition is the type of crystal structure of the film that grows on the substrate, whether amorphous, polycrystalline, or epitaxial. Ideally, we desire an epitaxial YSZ film. This provides the best condition for YBCO to grow on. However, achieving this is extremely difficult since the threshold between epitaxial and polycrystalline 
growth is very small. Actually, all deposition parameters decide whether a film grown is epitaxial or not. In the quest for epitaxy, we initially varied substrate temperatures between $225^{\circ} \mathrm{C}$ and $900^{\circ} \mathrm{C}$. From the literature, temperatures of $760^{\circ} \mathrm{C}-800^{\circ} \mathrm{C}$ were stated as being required to grow at least highly oriented (200) YSZ films on Si. For this reason the majority of the experiments were carried out at temperatures of $760^{\circ} \mathrm{C}$ to $900^{\circ} \mathrm{C}$.

Cooling after deposition was done either by turning of the heater and letting the heat dissipated naturally or slowly decreasing the temperature at a rate of $3-7^{\circ} \mathrm{C} / \mathrm{min}$ until at $500^{\circ} \mathrm{C}$ the heater was switched of. This was thought to allow some relaxation of the stress in the YSZ lattice and reduce the possibility of cracking. However, no significant effect was observed.

\subsection{YBCO Deposition}

The YBCO deposition was carried out by the pulsed laser ablation, which is essentially a plasma enhanced evaporation method. A Nd-YAG laser $(\lambda=355 \mathrm{~nm})$ is focused on a $2.54 \mathrm{~cm}$ diameter sintered $\mathrm{YBCO}$ target. As the laser beam hits the target photons are absorbed by the target causing a molten surface layer to form, which subsequently vaporizes. Further interaction of the beam on the vapor results in multi-photon ionization events that produce a plasma plume as shown in figure 6.3. The rate of evaporation is proportional to the laser fluence, as in the energy and volume of the plasma plume. 


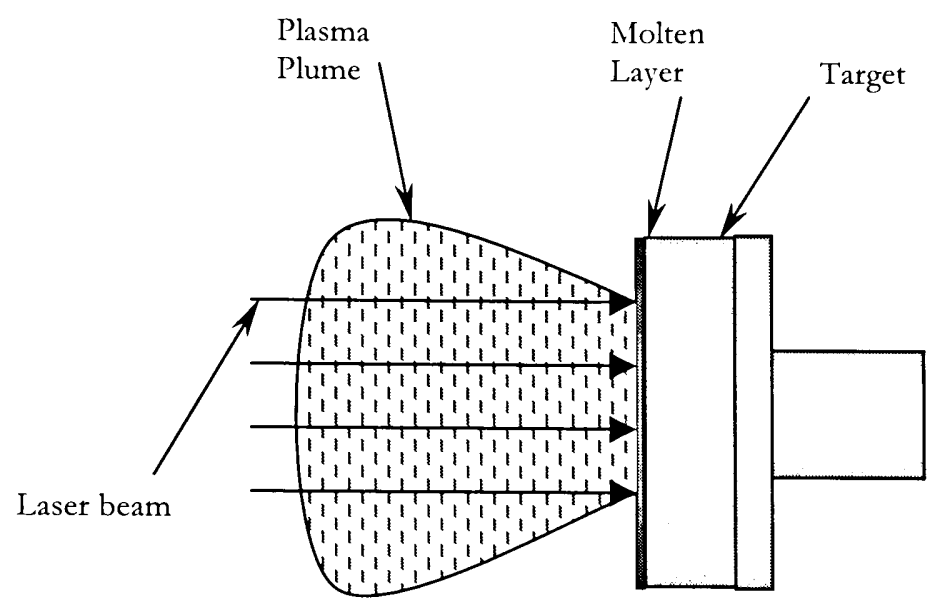

Figure 6.3 Laser Ablation

A substrate placed in the vicinity of the plume receives the evaporated particles where they nucleate and form a thin film. Due to the high photon energies involved, YBCO when vaporized loses oxygen and hence an oxygen flow rate is required to restore oxygen in the growing film.

Optimization of deposition parameters has been carried out in previous research $[65,66]$. The growth conditions were $500 \mathrm{mTorr}_{2}$ pressure and a substrate temperature of $780^{\circ} \mathrm{C}$. The laser was pulsed at a $10 \mathrm{~Hz}$ rate with energy of $120 \mathrm{~mJ} /$ pulse. The beam was focused to an $\sim 1 \mathrm{~mm}$ diameter spot which corresponds to an energy density of about 1.5 $\mathrm{J} / \mathrm{cm}^{2}$ on the target. The final film thickness after 30 minutes deposition time was about 200 nm. 


\subsection{Film Characterization}

After each YSZ deposition the films were analyzed by two methods. Ellipsometry was used to measure the thickness of the film and its index of refraction. The value of the refractive index is somewhat a measure of the film density. Denser films have higher refractive indices than do less dense films. Only a few films underwent this analysis and it was found that the equipment did not provide accurate enough readings.

$\mathrm{X}$-ray diffraction provides information about the structure of the film deposited. It tells us whether or not the film is crystalline and if it is, what orientation and how well organized it is.

\subsubsection{X-ray Diffraction}

A short explanation of $\mathrm{x}$-ray diffraction is given in this section since this was the primary method of analysis.

When x-rays are focused on a crystal at an angel of incidence $\theta$, the intensities of the reflections are analyzed. Crystals produce characteristic reflection peaks at specific angles of $\theta$, depending on the spacing of their inter-lattice planes. The information on values of $\theta$ that correspond to various crystals is usually stored in a database. After a scan is completed, the data is compared to the database and a plot is generated that shows the reflection intensity over a wide range of angles. By examining this $\theta-2 \theta$ plot, one can determine what crystal phases are present in the film. The greater the number of phases present, the greater the number of peaks in the plot. Since our ideal was to obtain epitaxial films, the degree of orientation can also be told from a phi-plot. The $\phi$ plot, also known as a texture map, is 
made by fixing the value of $\theta$ for a given crystal orientation, and analyzing the change in response while rotating the sample azimuthally. The range of rotation is from $\phi=0$ to $90^{\circ}$. A fully epitaxial film will produce peaks at discrete angles of $\theta$ and $\phi$ while partially oriented and random in-plane films produce rings and non-distinct plots. The rocking-curve is another plot that the degree of orientation may be gleaned. A particular peak is chosen and the width of the peak is measured according to the full width value at half the maximum intensity.

The x-ray analysis for our films were done by Dr. Patrica Stampe and Dr. Robin Kennedy at the National High Magnetic Fields Laboratory using a Philips Materials Research Diffractometer (MRD) PW3040 with a 4-circle goniometer and thin film optics. It uses Cu K-alpha radiation with a $\lambda=1.5604 \AA$.

\subsubsection{Resistance-Temperature Measurement}

Resistance measurements were carried out on the YBC0/YSZ/Si samples using the four-point probe method shown in figure 6.4.2.

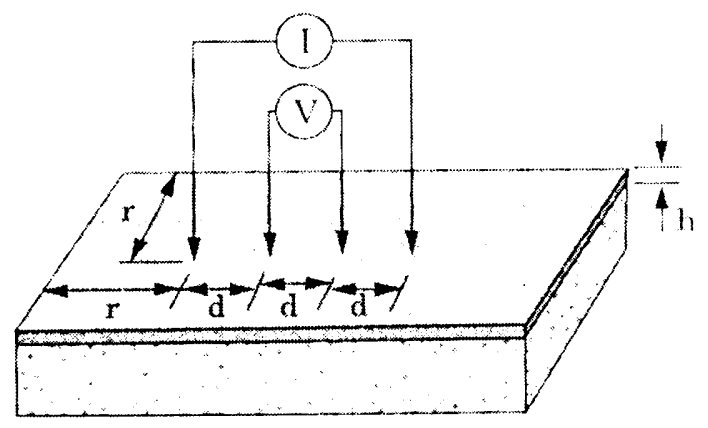

Figure 6.4.2 Four-point Probe Configuration 
The advantage of using the four-point probe technique is that the influence of the contact resistance is kept at a minimum. When a simple measurement of resistance using two probes attached to the sample is carried out, the resistance of the contact points included in the measurement. This may not have a great effect when measuring large resistances, since the contact resistance is usually very small. However, when measuring the extremely small resistances of superconductors such as YBCO, the contact resistance can dominate and mask small changes in the resistance of the sample itself.

As the current flows between the two terminals shown in the figure, any resistance will cause a voltage drop. The voltage probes shown in the figure record this voltage drop. The resistance is then obtained by calculating the ratio of voltage to current. An HP 3365A impedance meter with the four-probe feature was used to perform this task.

A helium closed-cycle refrigeration unit was used to provide cooling of the samples. Samples were mounted inside a vacuum enclosure and resistance readings were taken for temperature ranges of $20-270 \mathrm{~K}$.

Five characteristics are of interest when measuring the resistance of YBCO film.

1. The room temperature resistance: For a $300-\mathrm{nm}$ thick YBCO film it is usually on the order of $10 \Omega$.

2. Slope of curve: When a superconductor is cooled the resistance steadily decreases. The slope of this curve should be smooth and have a positive slope. 
3. Critical temperature: This is the temperature at which the resistance becomes zero. For good quality YBCO, this value should be in the range of $87-92 \mathrm{~K}$

4. Transition width: When the superconductor enters the superconducting state, the resistance drops sharply to zero. The difference in the temperature at which this phenomena starts to occur and the temperature at which the resistance actually becomes zero is called the transition width. YBCO that is single phase and highly oriented should ideally have a transition width of $5 \mathrm{~K}$ or less.

5. Number of Slopes: YBCO films that have grown with multiple phases can be detected by the presence of more than one slope in the transition region of the resistance-temperature curve. The presence of one slope is ideal. 
In this section we tabulate the experiments performed and present the results in the form of film characterization analysis done on the films. The primary tool was $\mathrm{x}$-ray diffraction, used to determine what film structure has been grown and its degree of orientation with the substrate. Other methods employed include ellipsometry, to determine index of refraction and film thickness, and Resistance versus temperature measurements of the final YBCO film grown on the YSZ buffered Si.

\subsection{Experiments: Set 1}

In table 7.1 below, the first set of experiments that were carried is tabulated.

\begin{tabular}{|l|l|l|l|}
\hline Sample\# & 1 & 2 & 4 \\
\hline Substrate & $\mathrm{Si}$ & $\mathrm{Si}$ & $\mathrm{Si}$ \\
\hline Target & YSZ-A & YSZ-A & YSZ-A \\
\hline RF Power(W) & 100 & 100 & 100 \\
\hline Argon Flow Rate (sccm) & 4 & 4 & 4 \\
\hline Oxygen Flow Rate (sccm) & 0.8 & 0.8 & 0.8 \\
\hline $\begin{array}{l}\text { Sputtering Pressure } \\
\text { (mTorr) }\end{array}$ & $10-11$ & $10-11$ & $10-11$ \\
\hline $\begin{array}{l}\text { Pre-Sputter Time (min) } \\
\text { Temperature (C) }\end{array}$ & 10 & 10 & 10 \\
\hline $\begin{array}{l}\text { Target-substrate Distance } \\
\text { (cm) }\end{array}$ & 6.35 & 6.35 & 6.35 \\
\hline Duration (min) & 120 & 120 & 120 \\
\hline
\end{tabular}

Table 7.1 Experimental Set 1 
In this set of experiments, we started out by choosing a nominal sputtering pressure of $10 \mathrm{~m}$ Torr. This was established using an $\mathrm{Ar}: \mathrm{O}_{2}$ ratio of 5:1 with the flow rates as shown in the table. Deposition times were held constant at $2 \mathrm{hrs}$ as was the target-substrate distance and R.F. power was used. The only parameter varied was the temperature. We needed to see what range of temperature gave a particular crystal structure when analyzed by x-ray diffraction. The target used, denoted YSZ-A, refers to the low oxygen content YSZ target previously mentioned.

For sample \#1, grown at $440^{\circ} \mathrm{C}$, the $\mathrm{x}$-ray diffraction appears in figure 7.1 (a). The presence of a peak at approximately $27.9^{\circ}$ indicates the presence of (111) oriented YSZ. We can tell it has a relative high orientation since it is the strongest peak. The rocking curve pictured in figure 7.1 (b) is a measure of how well the alignment is. At its best, a rocking curve's full width at half maximum (FWHM) should be $<1^{\circ}$, indicating full crystal alignment. Our sample had a measured FWHM of $8^{\circ}$ indicating that the film's in-plane orientation is random.

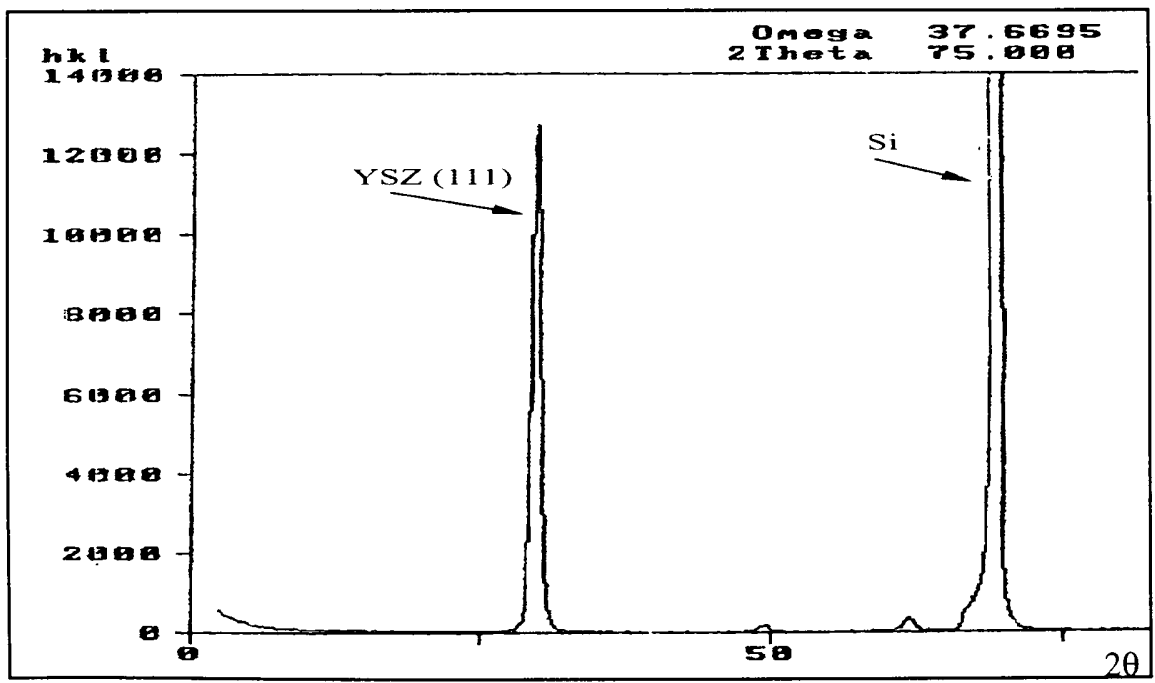

Figure 7.1(a) X-ray spectrum of sample \#1 $\left(440^{\circ} \mathrm{C}\right)$ 


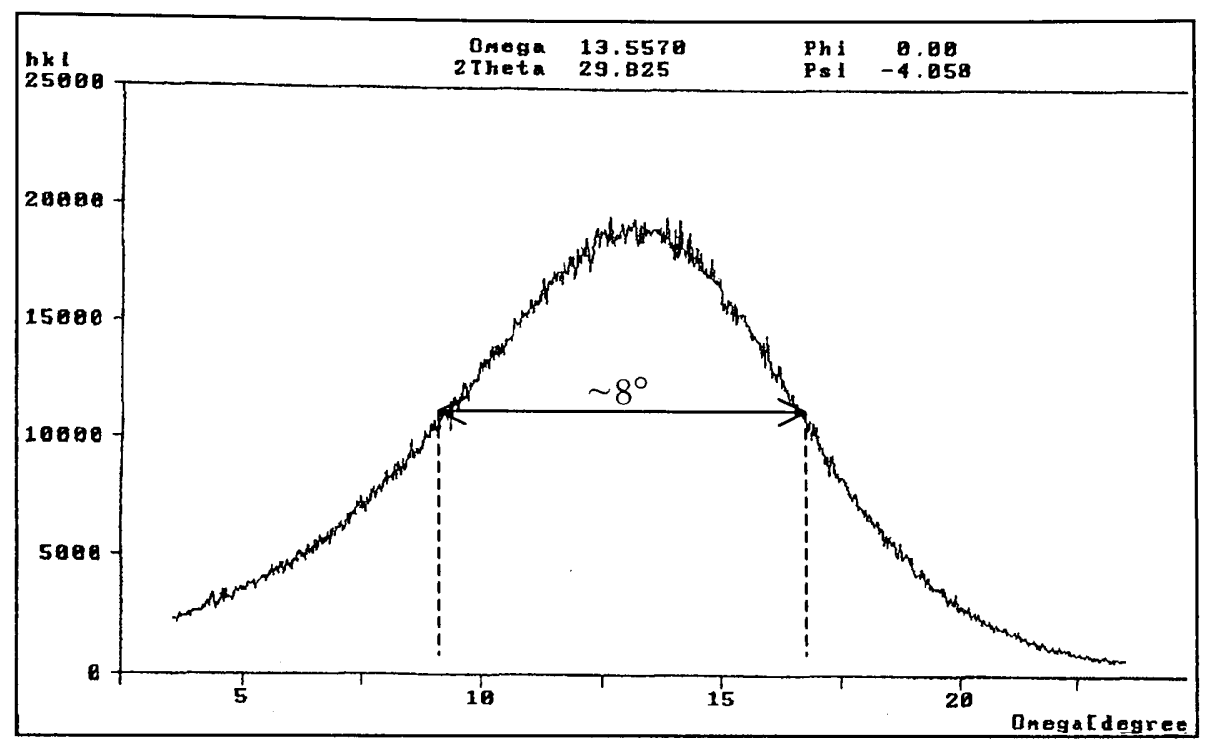

Figure 7.1(b) Rocking Curve of sample \#1

In sample \#2, the temperature was decreased to $225^{\circ} \mathrm{C}$. The appearance of another peak besides the (111) reflection is evident in figure 7.1(c).

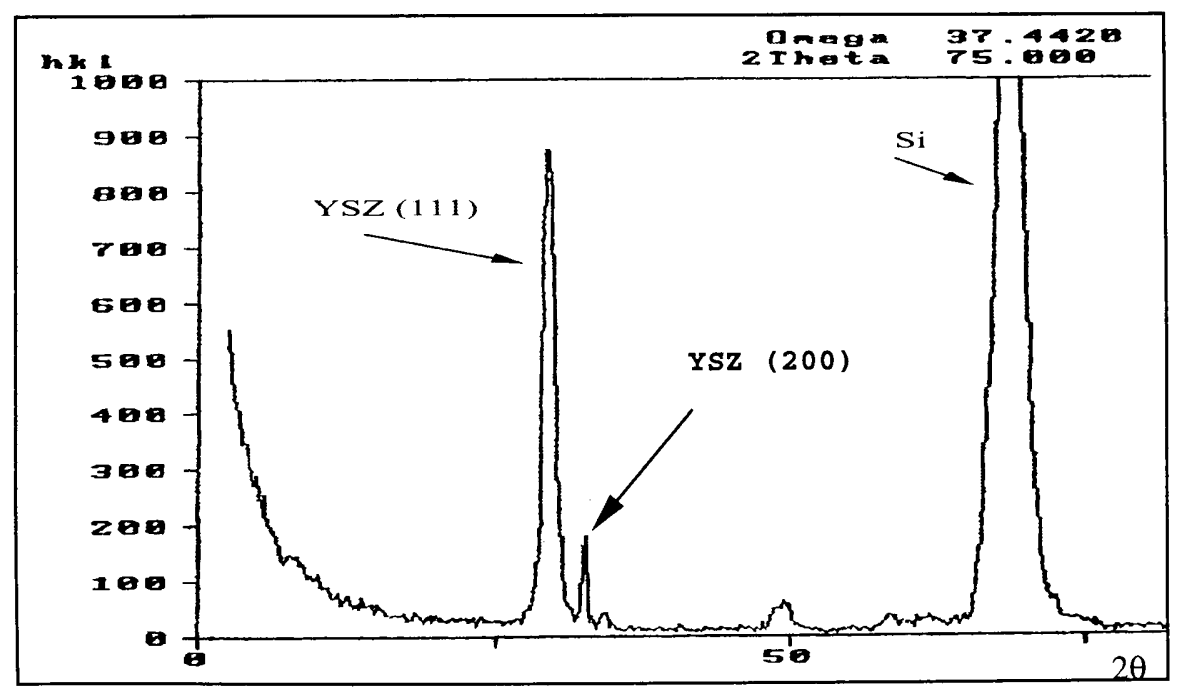

Figure 7.1(c) X-ray spectrum of sample \#2 $\left(225^{\circ} \mathrm{C}\right)$ 
This peak is measured at $28.2^{\circ}$ and corresponds to the (200) reflection of YSZ. The appearance of this phase suggests that at low temperature several orientations may exist corresponding to an extremely polycrystalline film. An increase of temperature above $500^{\circ} \mathrm{C}$ yielded an increase in the presence of the (200) reflection.

Sample \#4 was made at $700^{\circ} \mathrm{C}$ and the resulting x-ray scan and rocking curve are shown in figures 7.1(d) and 7.1(e). We note that although we get strong (200) oriented films, the rocking curve of $11^{\circ}$ indicates that the crystal orientation is not perfect.

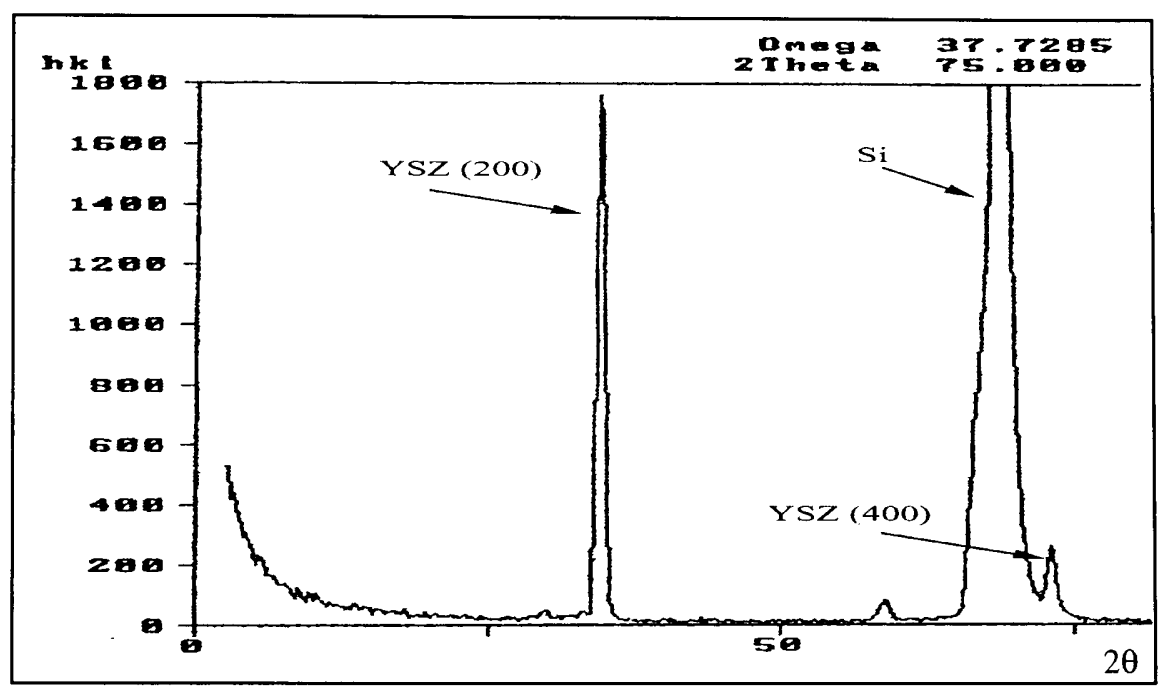

Figure 7.1(d) X-ray scan of sample \#4 


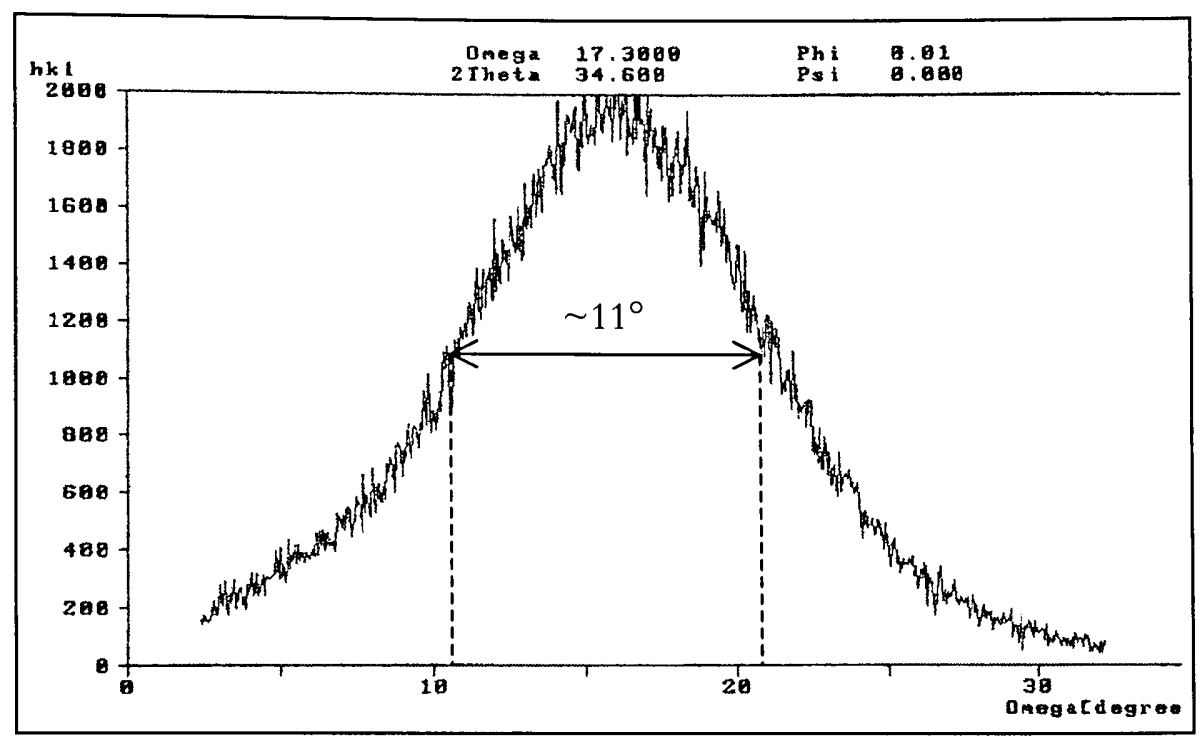

Figure 7.1(e) Rocking curve for sample \#4

From this first set of experiments we verified that substrate temperatures of at least $700^{\circ} \mathrm{C}$ was necessary to obtain (200) oriented YSZ films. Since our ultimate goal is to create substrates suitable for YBCO film growth, deposition of YBCO films was carried out on these samples and the resistance measured by the method explained in section 6.4.2. The results for sample \#1 and \#2 are shown in figures 7.1(f) and 7.1(g). 


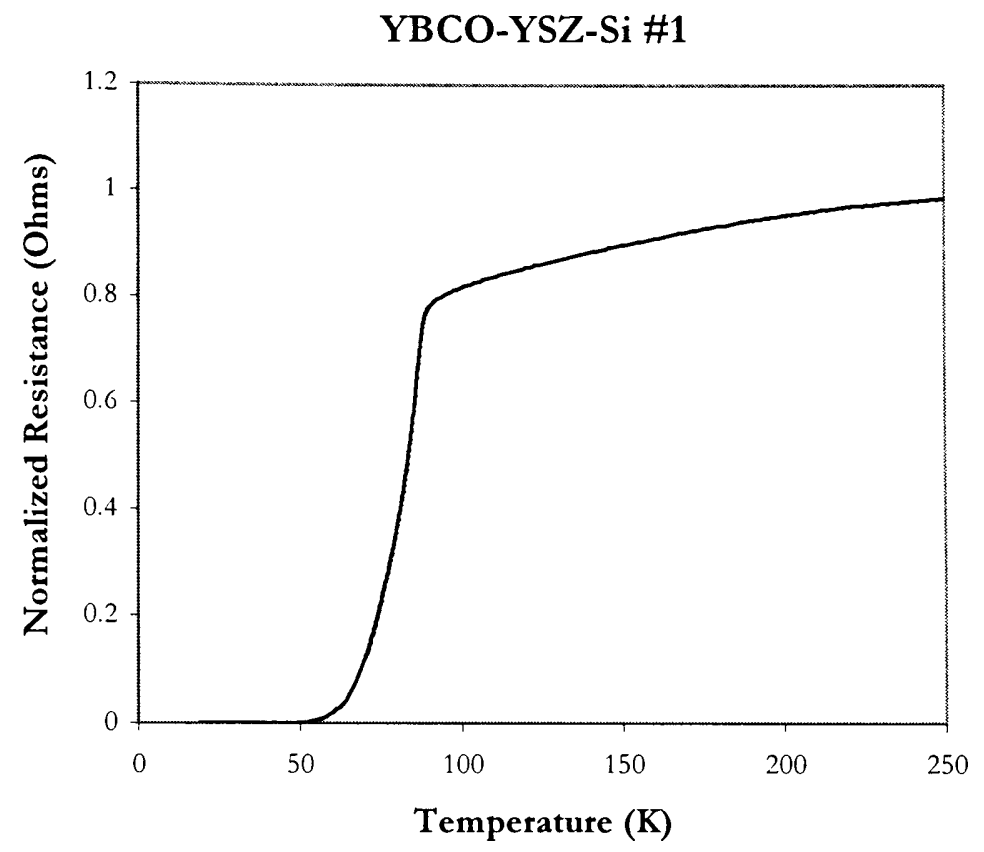

Figure 7.1(f) $\quad \mathrm{R}$ vs. $\mathrm{T}$ for sample \#1

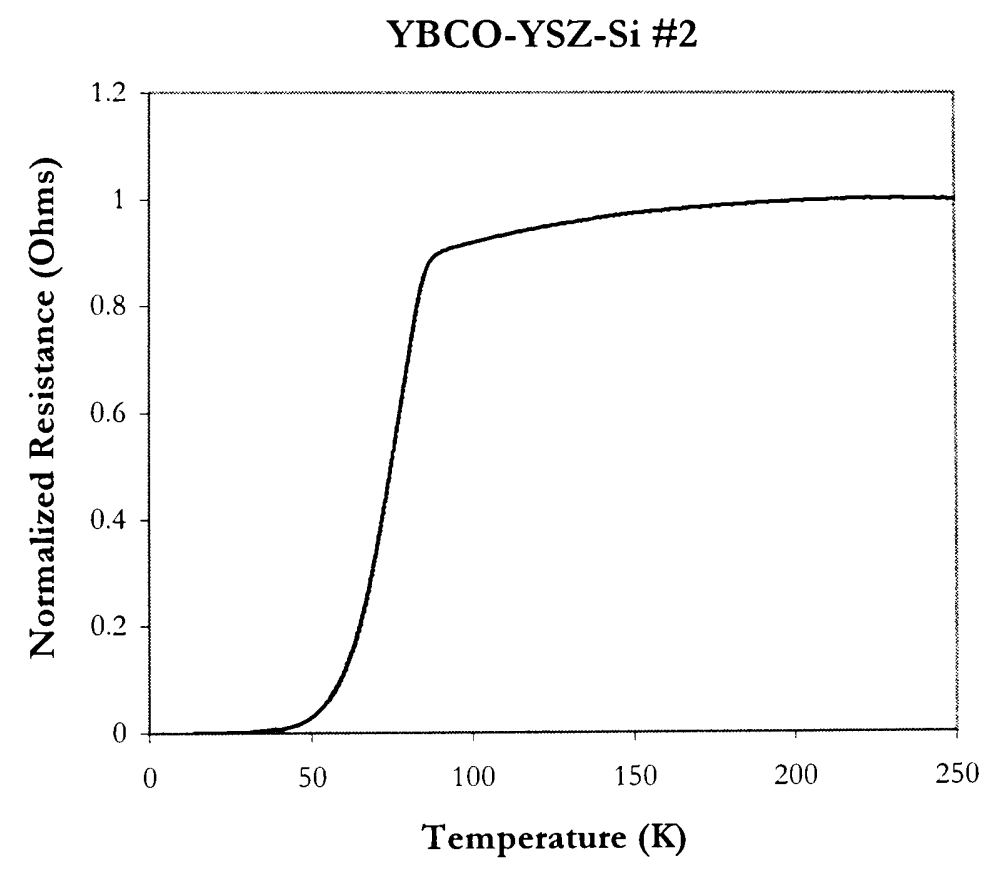

Figure 7.1(g) $\mathrm{R}$ vs. $\mathrm{T}$ for sample \#2 
We see that the $\mathrm{T}_{c(R=1)}$ of the YBCO film is lower on sample \#2, the sample that had YSZ deposited at $225^{\circ} \mathrm{C}$, than sample \#1 which was done at $440^{\circ} \mathrm{C}$. The appearance of multiple phases in the $\mathrm{x}$-ray scan of sample \#2 suggests that the random, polycrystalline nature of the film did not provide a good surface for the YBCO to grow on. Sample \#1, which had good (111) oriented YSZ, provided better results. The $\mathrm{T}_{c}$ of sample \#2 was measured to be $23 \mathrm{~K}$ while sample \#1 had a $\mathrm{T}_{\mathrm{c}(\mathrm{R}=0)}$ of $47 \mathrm{~K}$.

The $\mathrm{R}$ vs. $\mathrm{T}$ result for sample \#4 is not shown since it did not exhibit a critical temperature at all. When this happens, typically the data collection is turned off. Even though the sample had a good (200) oriented film, YBCO did not grow properly enough to be superconducting. Initially it was thought that (200) oriented YSZ grown on Si was not suitable for YBCO film growth. However, this notion conflicted greatly the literature. A more plausible explanation is that since a constant oxygen flow rate was being maintained during deposition, the Si substrates were more likely to be oxidized at the high deposition temperature of $700^{\circ} \mathrm{C}$. Too great of an oxidation of the silicon surface can lead to films that are porous with low packing density. YBCO deposited on such surfaces diffuse through the porous YSZ film and readily reacts with the $\mathrm{Si}$, which destroys its superconducting properties.

Our goal was to try and improve the film quality of the (200) YSZ films. (200) orientation is desired because it is the first step in trying to obtain a fully epitaxial (200) II (100) relation between YSZ and Si. 


\subsection{Experiments: Set 2}

\begin{tabular}{|l|l|l|l|}
\hline \multicolumn{1}{|c|}{ Sample\# } & 8 & PDC-1 & PDC-5 \\
\hline Substrate & Si & Si & Si \\
\hline Target & YSZ-A & YSZ-A & YSZ-A \\
\hline Power(W) & $100(\mathrm{RF})$ & $100($ PDC) & $100($ PDC) \\
\hline Argon Flow Rate (sccm) & 4 & 4 & 40 \\
\hline Oxygen Flow Rate (sccm) & 0.8 & 0.8 & $\boldsymbol{8}^{*}$ \\
\hline $\begin{array}{l}\text { Sputtering Pressure } \\
\text { (mTorr) }\end{array}$ & $10-11$ & $10-11$ & $36-37$ \\
\hline Pre-Sputter Time (min) & 10 & 10 & 10 \\
\hline Temperature (C) & 800 & 800 & 800 \\
\hline $\begin{array}{l}\text { Target-substrate Distance } \\
\text { (cm) }\end{array}$ & 6.35 & 6.35 & 6.35 \\
\hline \begin{tabular}{l} 
Duration (min) \\
\hline
\end{tabular} & 120 & 120 & 60 \\
\hline
\end{tabular}

Table 7.2 Experimental Set 2

In this set of experiments, we sought to find out what parameters we had to change in order to obtain good quality YSZ (200) films grown on Si. Sample \#8 was the best result gotten using R.F. power and high temperature. All previous samples from \#3 to \#7 yielded poor results. The YSZ film of sample \#8 grew with (200) orientation but random in plane. This is shown by figure 7.2(a) and 7.2(b)

\footnotetext{
* Oxygen flow rate not held constant
} 


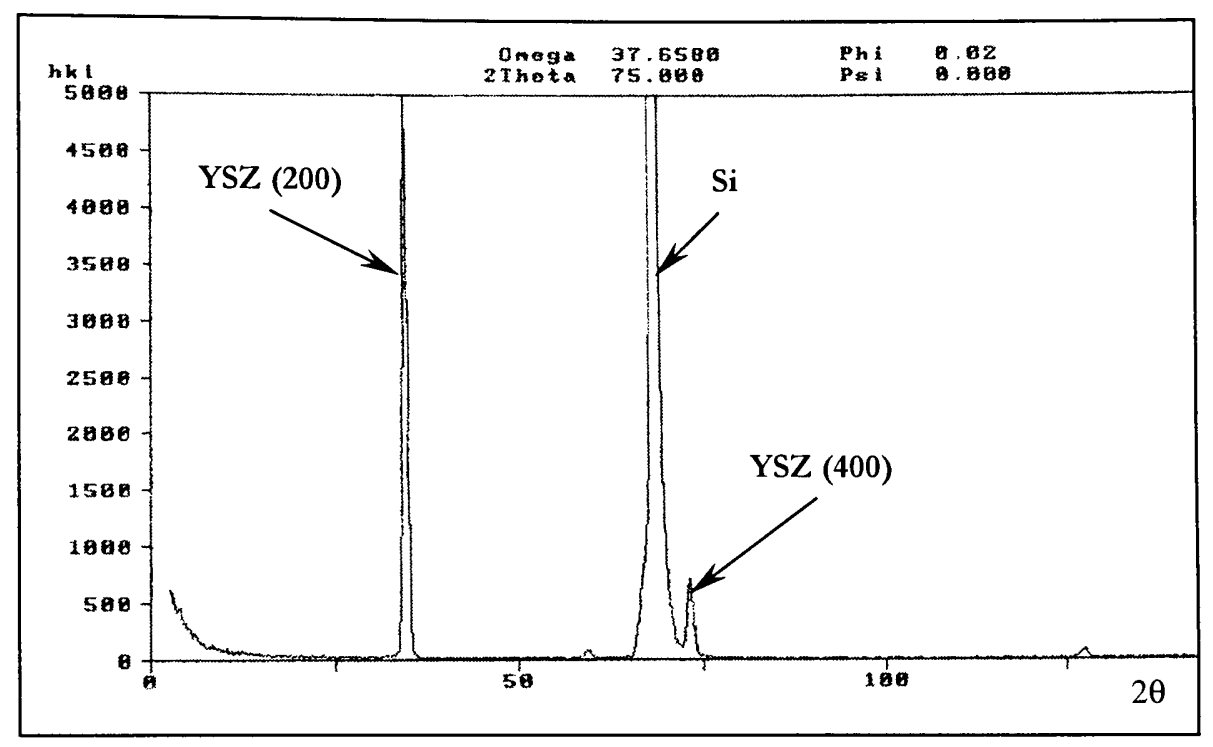

Figure 7.2 (a) X-ray scan of sample \#8

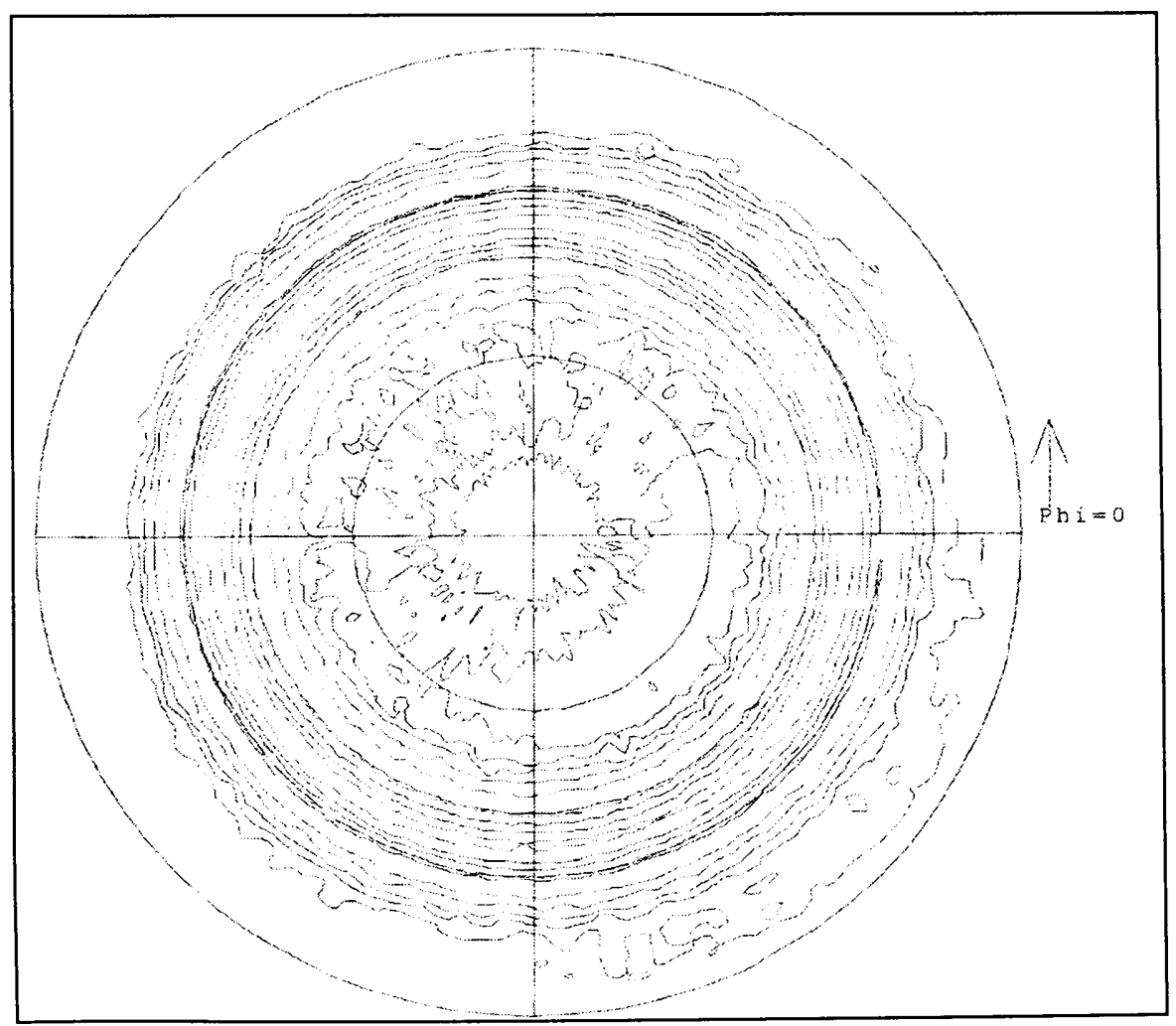

Figure 7.2(b) Phi-plot of sample \#8 
The unorganized patterns produced on the phi-plot suggest that the YSZ film had a high degree of disorientation. YBCO deposited on this sample measured a $T_{c(R=0)}=\sim 15 \mathrm{~K}$. As shown in figure 7.2 (c), the curre does not demonstrate a positive slope. The transition has multiple slopes over a relatively large transition width. These two observations are a clear indicator that the YBCO had a hard time growing on the surface of sample \#8. Howerer up to this point, this was the only sample done with R.F power to even exhibit some suggestion of a superconductive transition.

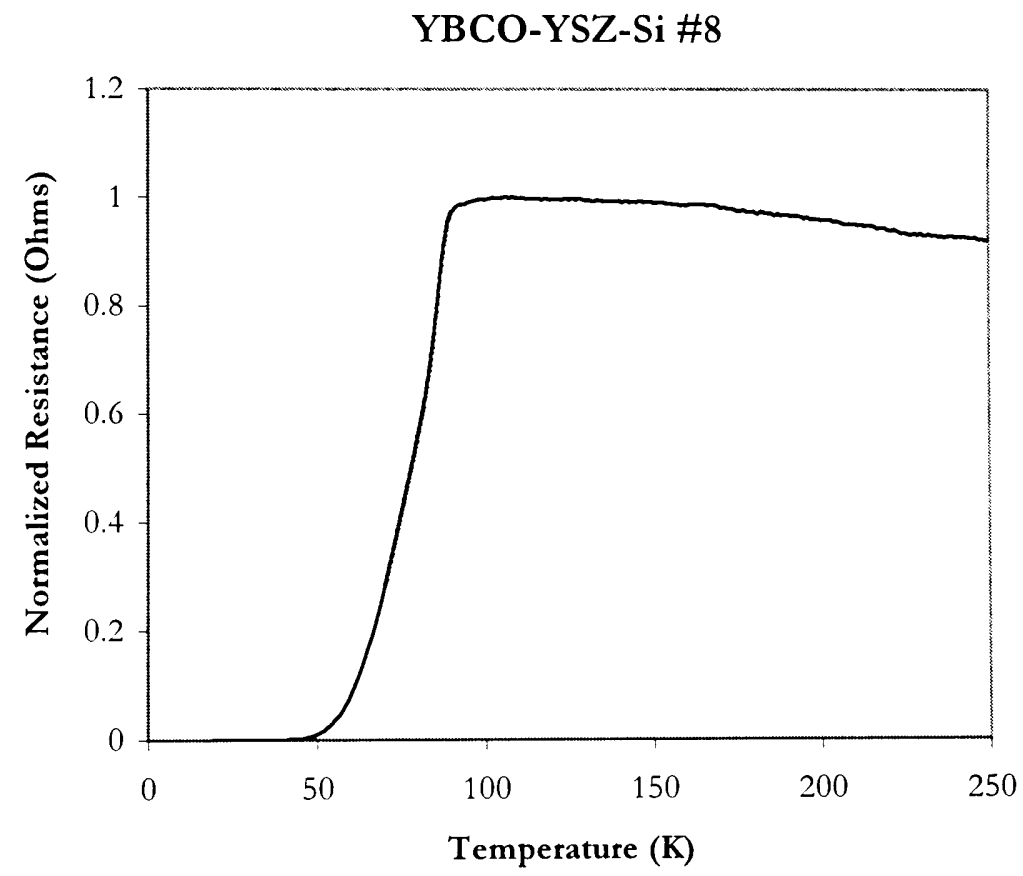

Figure 7.2(c) $\quad R$ vs. T of sample \#8

Now, we decided to use pulsed D.C. power instead, to see if any improvements could be made. It was thought that if higher deposition rate per second, could be maintained, then not only would the films' density increase but also the effective oxidation 
of the Si surface could be reduced by quicker YSZ coverage of the substrate. The first sample done in such a manner was sample \#PDC1. Besides the substitution of pulsed D.C. power, no other parameters were changed from sample \#8. The results obtained were astonishing. The $\mathrm{x}$-ray scan is shown in figure $7.2(\mathrm{~d})$.

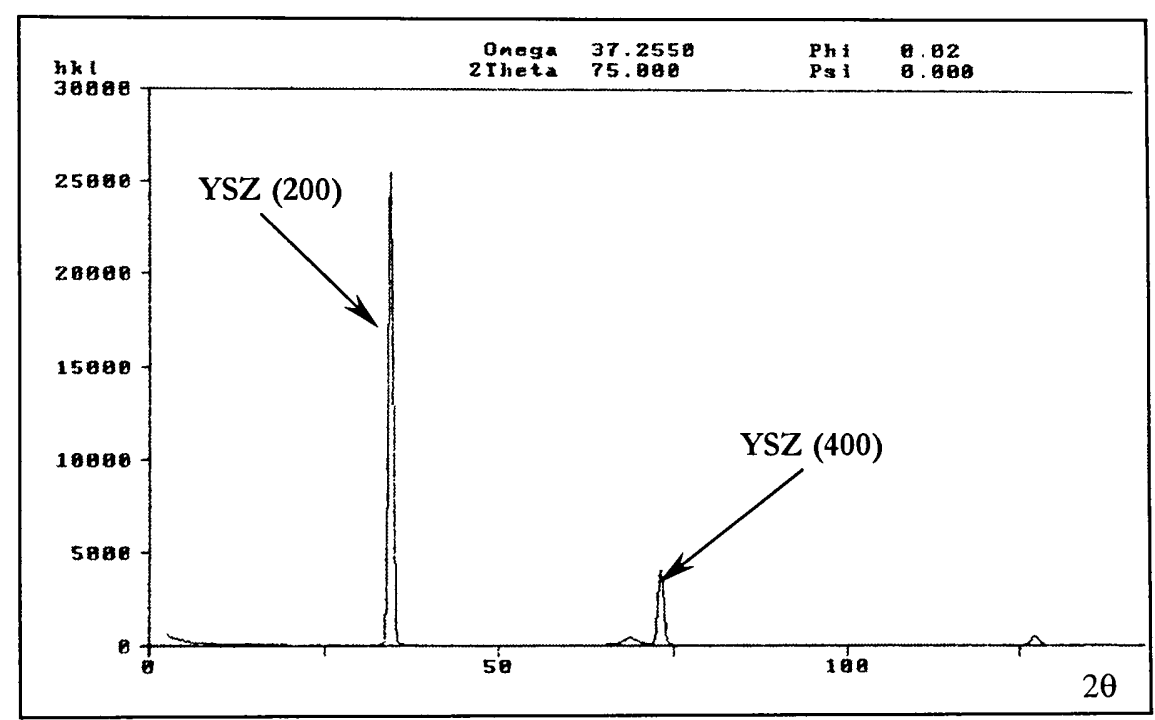

Figure 7.2(d) X-ray scan for sample \#PDC1

The first thing to notice on this $\mathrm{x}$-ray scan is that the relative intensity of the (200) peak is extremely high. The y-axis of the scan shows the peak intensity at 25,000 . Relative to the previous scans this is two times higher than (111) film and 10 times higher than the previous (200) oriented films. The increased intensity represents a stronger presence of the (200) orientation. This result was remarkable in that although the same operating conditions were set, as in sample \#8, the use of pulsed D.C. power made a huge difference. The phi plot shown in figure 7.2(e), illustrates the sample as being randomly oriented, however not as 
much as sample \#8. This suggested the possibility of oxygen still interacting with the Si surface, causing disorientation in the YSZ growth.

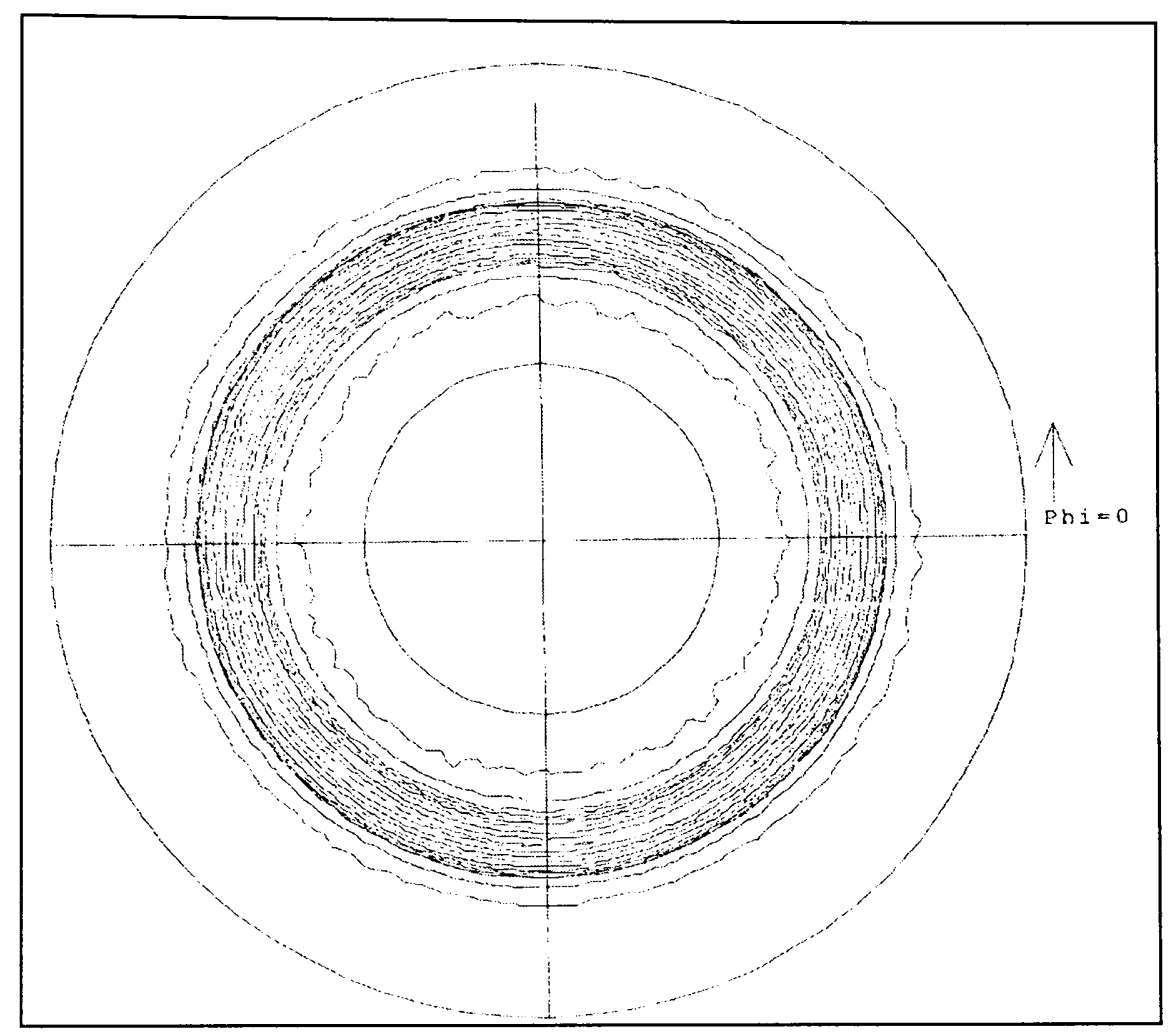

Figure 7.2(e) Phi-plot of sample \#PDC1

The $R$ vs. $T$ results are slightly better, with the sample showing a $T_{c(R=0)}=\sim 50 \mathrm{~K}$ as shown in figure $7.2(\mathrm{f})$.

The next thing we had to try was to modulate the oxygen flow and see if different results were produced. Sample \#5 represents this experiment. 
YBCO-YSZ-Si-PDC \#1

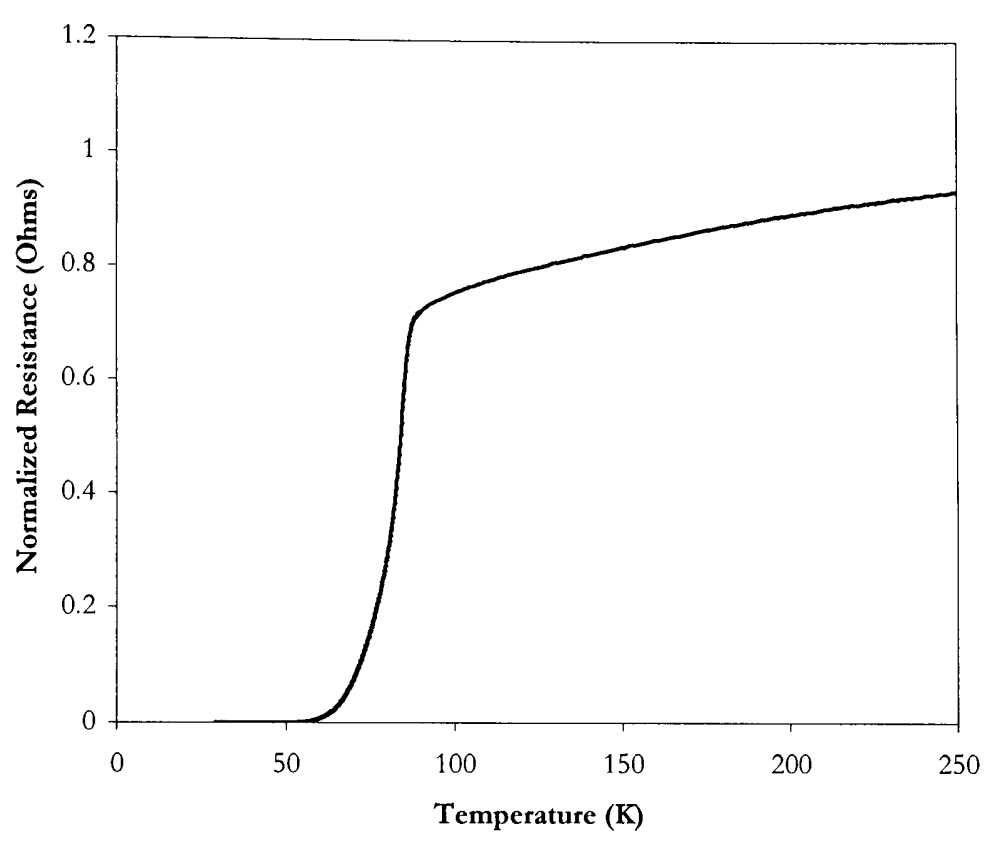

Figure 7.2(f) $\quad \mathrm{R}$ vs. $\mathrm{T}$ for sample \#PDC1

For the first 30 seconds during sample \#PDC5's deposition, the oxygen flow was ramped from 0 to $8 \mathrm{sccm}$. This flow was kept constant for 1 minute after which it was turned off. After 20 minutes deposition time the oxygen flow was again initiated with the 30 -second ramp time and kept constant for the rest of the 60 -minute run. It was believed that such a method would reduce the substrate's average time of exposure to oxygen.

We decided to decrease the deposition time for two reasons. First, a shorter deposition time could mean less time for the substrate to be in contact with oxygen. Second, the $1700+\AA$ thick films that were being produced before could have been too thick. The large difference in the thermal expansion coefficients of Si and YSZ (see section 4.1-4.2) 
causes the YSZ film to cool faster than the Si substrate. This leads to tensile stress in the film causing it to crack. Deposition times were cut by half in order to obtain thinner films.

Another change that we made was to raise the sputtering pressure but keeping the Ar: $\mathrm{O}_{2}$ ratio the same. By doing this, the oxygen and ion effects on the substrate could be reduced.

This proved to be the turning point in our research. From the x-ray scan in figure 7.2(g) we see that the film has high (200) crystallinity. The (200) peak had a relative intensity of 35,000 counts, the highest response obtained. The phi-plot in figure $7.2(\mathrm{~h})$ shows a film at is almost $100 \%(200)$ oriented. There still is slight disorganization representing a very small amount of random crystal alignment.

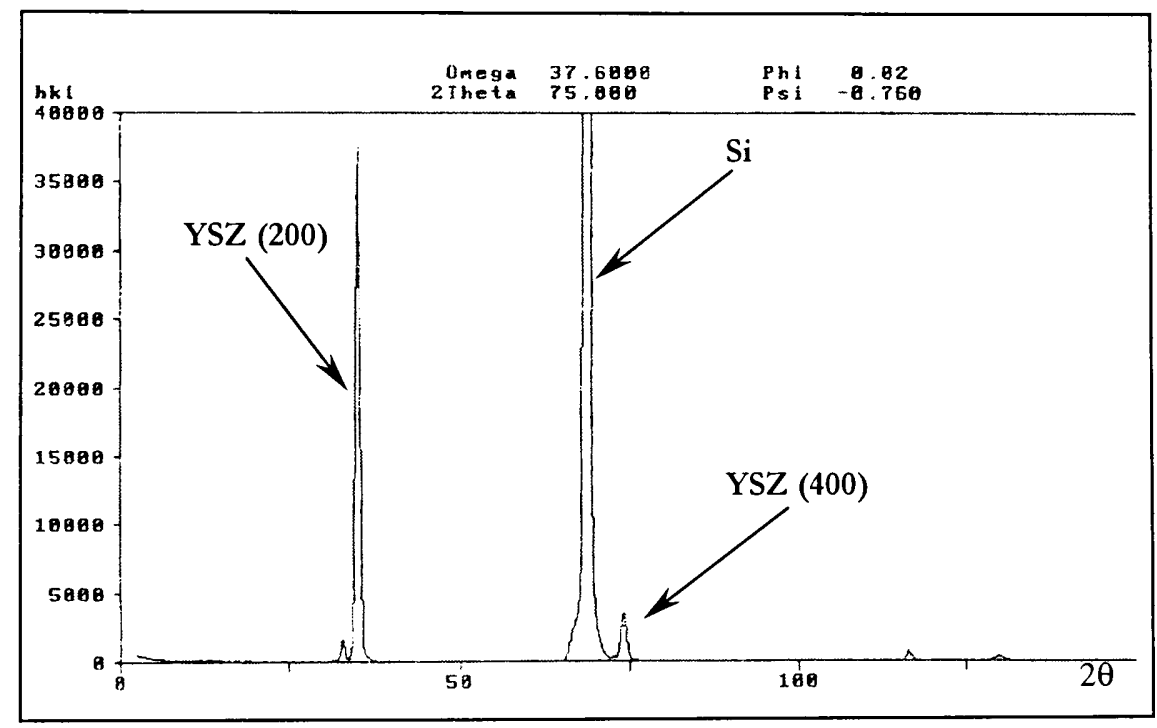

Figure 7.2(g) X-ray scan of sample \#PDC5 


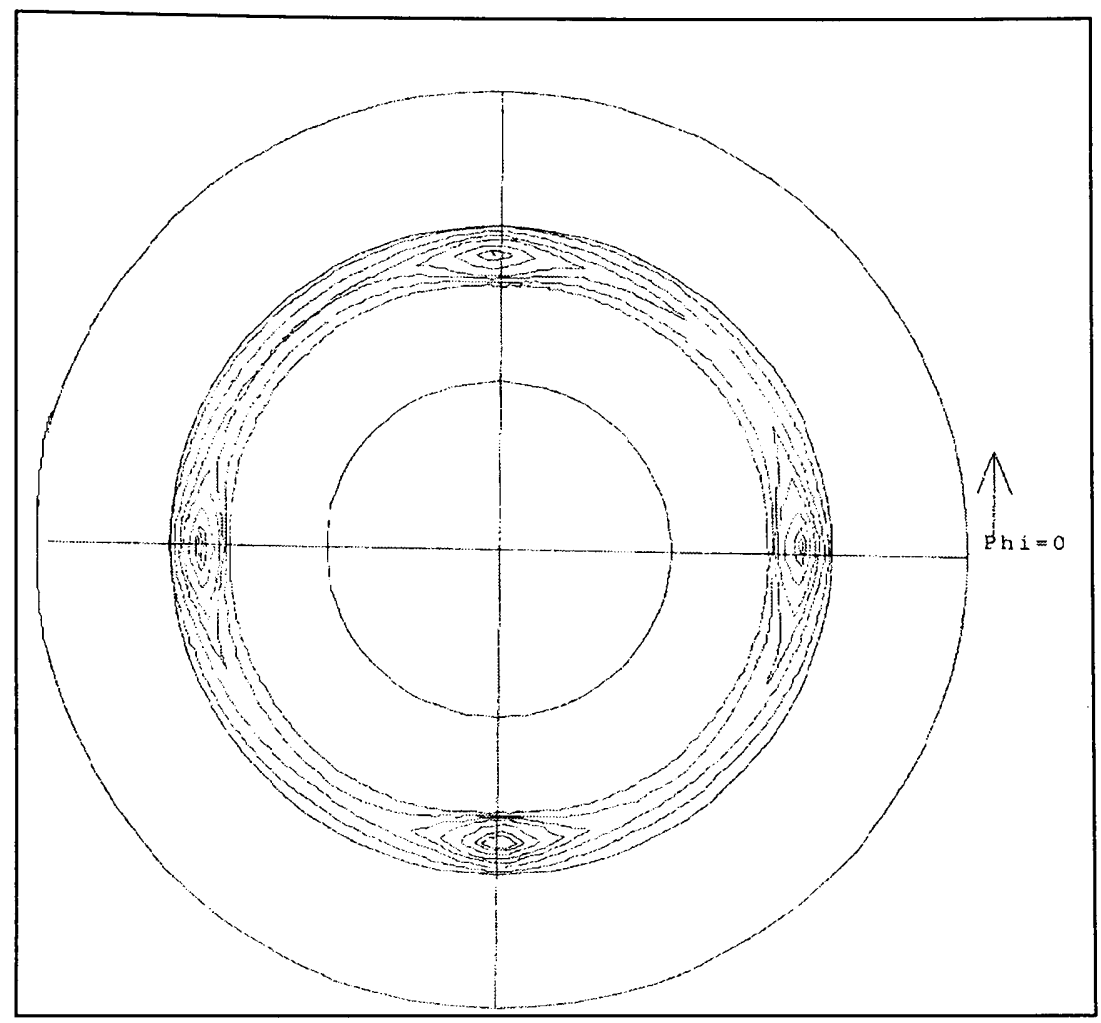

Figure 7.2(h) Phi-plot of sample \#PDC5

The formation of circular rings as shown, suggests near full epitaxial formation, with the film being partially oriented in plane.

This sample also had YBCO deposited on it and the resistance measured. Figure 7.2(e) shows the resistance-temperature plot. It is obvious that the critical temperature is much higher than previous samples. This sample had a $T_{c}=71 \mathrm{~K}$ and also had a much narrower transition width. Previous samples had transition widths of $\sim 25-30 \mathrm{~K}$ while this one had only a $\sim 18 \mathrm{~K}$ transition width, suggesting that YBCO growth on the near epitaxial YSZ film surface was improved. 
YBCO-YSZ-Si \#PDC5

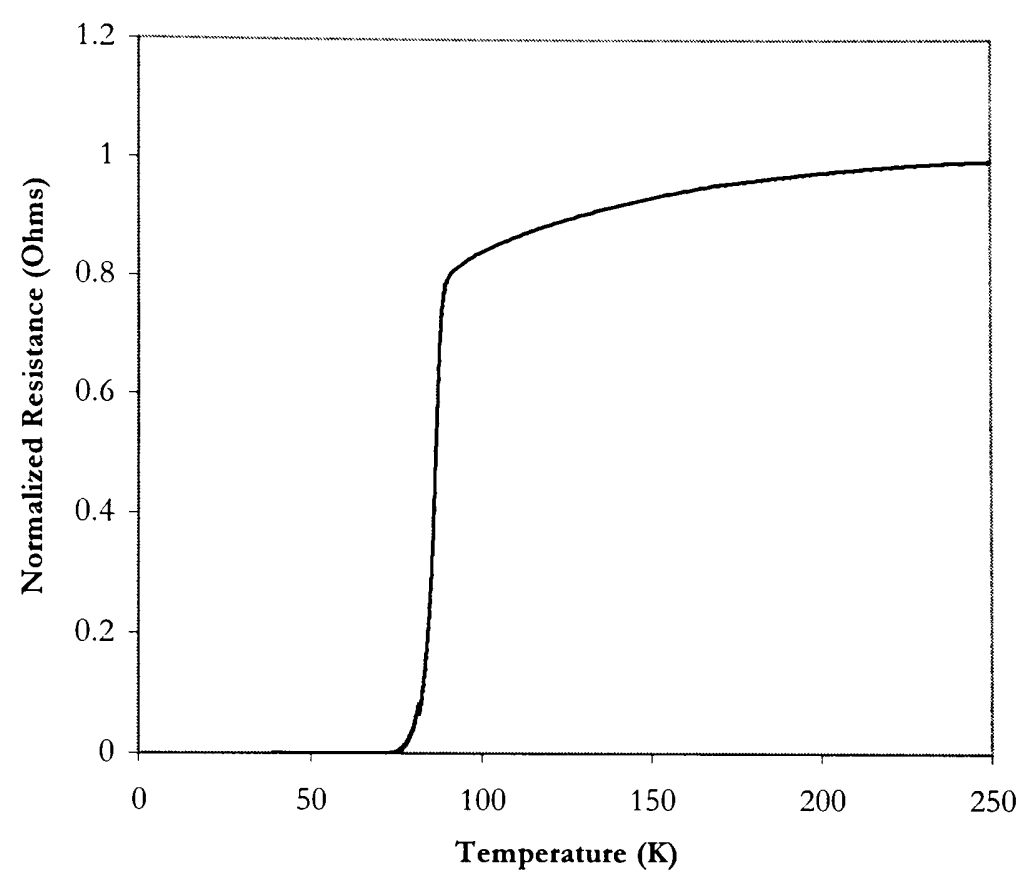

Figure 7.2(i) R vs. T of sample \#PDC5

This result marked a rise of $10 \mathrm{~K}$ in the $\mathrm{T}_{\mathrm{c}}$ obtained from previous samples. Clearly the combination of a shorter deposition time (thinner films) with control of $\mathrm{O}_{2}$ represented better operating conditions for depositing YSZ films. The task that followed was to reproduce these results or even improve them. 


\subsection{Experiments: Set 3}

The next set of experiments is shown in table 7.3.

\begin{tabular}{|l|l|l|l|}
\hline \multicolumn{1}{|c|}{ Sample\# } & PDC-12 & PDC-13 & RFN02 \\
\hline Substrate & $\mathrm{Si}$ & $\mathrm{Si}$ & $\mathrm{Si}$ \\
\hline Target & YSZ-A & YSZ-A & YSZ-B \\
\hline Power(W) & 80 (PDC) & 80 (PDC) & 100 (PDC) \\
\hline Argon Flow Rate (sccm) & 50 & 50 & 50 \\
\hline Oxygen Flow Rate (sccm) & $4^{*}$ & $4^{*}$ & - \\
\hline $\begin{array}{l}\text { Sputtering Pressure } \\
\text { (mTorr) }\end{array}$ & $26-27$ & $13-15$ & $14-15$ \\
\hline Pre-Sputter Time (min) & 10 & 10 & 10 \\
\hline Temperature (C) & 800 & 830 & 830 \\
\hline $\begin{array}{l}\text { Target-substrate Distance } \\
\text { (cm) }\end{array}$ & 6.35 & 6.35 & 6.35 \\
\hline Duration (min) & $\mathbf{3 0}$ & $\mathbf{1 5}$ & $\mathbf{3 0}$ \\
\hline
\end{tabular}

Table 7.3 Experimental Set 3

Having decided that pulsed D.C power produced better results, various methods of controlling the $\mathrm{O}_{2}$ flow rate had implemented with samples \#PDC6 - \#PDC12. However, there was little or no change in the $T_{c}$ of these samples. The only change was in the YSZ films. No longer were we able to get near epitaxial films. There are many reasons for this; the main one being that there is extremely small tolerance in the deposition parameters that produce epitaxial films. Any small deviation will result in films being polycrystalline. Another possible reason is that the substrate heater broke and was replaced by a smaller

\footnotetext{
* Oxygen flow rate not held constant
} 
heater. This heater provided us with temperatures of up to $900^{\circ} \mathrm{C}$ but radiated much less heat to the surrounding chamber volume. The result of this was that overall sputtering pressures were lower with the same amount of gas flow. This anomaly skewed the entire parameter space and we were presented with a new challenge.

With sample \#PDC12 we decreased the $\mathrm{O}_{2}$ partial pressure to $8 \%$ instead of $20 \%$ to further minimize the effects on the substrate. It was modulated in a similar manner to sample \#PDC5 but the flow was instead pulsed on and off, in 120 second intervals, for the duration of the deposition. The Ar flow rate was increased to $50 \mathrm{sccm}$ in order to maintain a sputtering pressure of $27 \mathrm{~m}$ Torr. The deposition rate was lowered to 30 minutes to obtain even thinner films. Only the resistance-temperature plot (figure 7.3(a)) is presented here because the $x$-ray scans were unavailable. However, the $R$ vs. $T$ plot shows us that the $T_{c}$ increased slightly to $75 \mathrm{~K}$.

YBCO-YSZ-Si \#PDC12

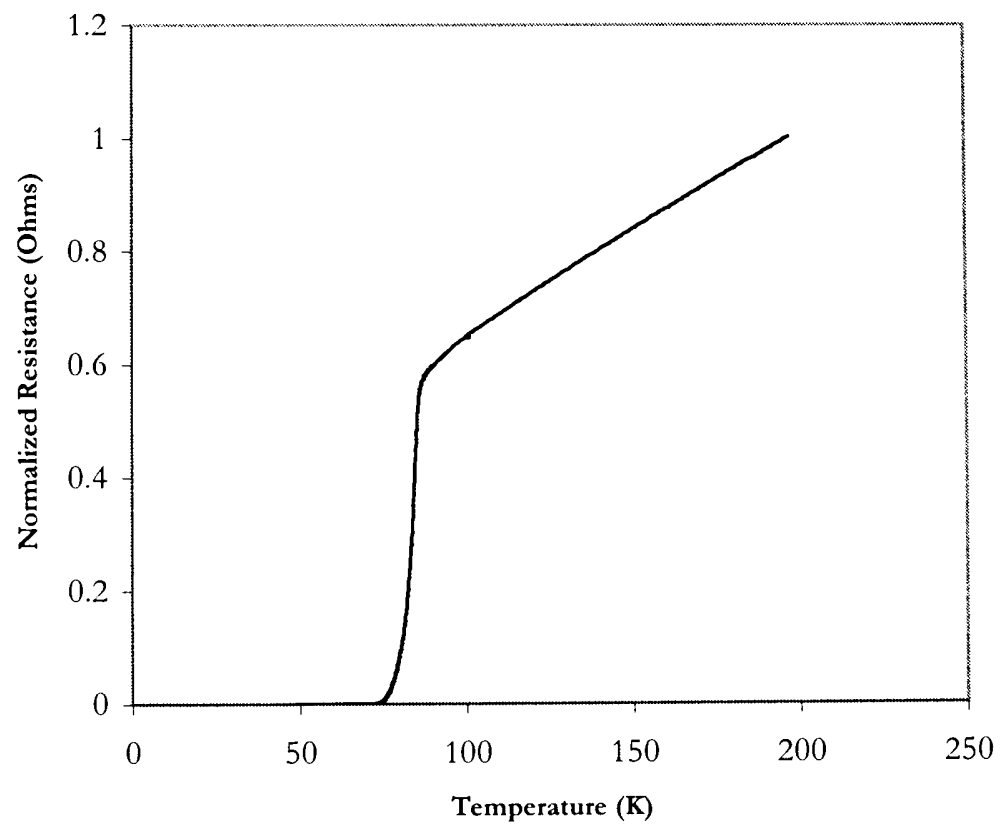

Figure 7.3(a) R vs. T plot for sample \#PDC12 
Sample \#PDC13 represents the first sample that was used with the new heater. Although the $\mathrm{Ar}$ and $\mathrm{O}_{2}$ flow rate were the same the sputtering pressure decreased to 15 mTorr. Knowing that we would have to start a new search for operating parameters with the new heater, we decided to start with the same gas flow rates to see the result. The only other changes that were made included raising the deposition temperature to $830^{\circ} \mathrm{C}$ and decreasing the deposition time to 15 minutes. By raising the temperature, the deposited particles could gain greater thermal energy thereby increasing the surface diffusion and possibly lead to better film formation. The resistance-temperature plot shown in figure 7.3(b) demonstrates an increase in the $T_{c}$ to $80 \mathrm{~K}$. This indicated that thinner films, on the order of $40-50 \mathrm{~nm}$ seemed to provide the best results.

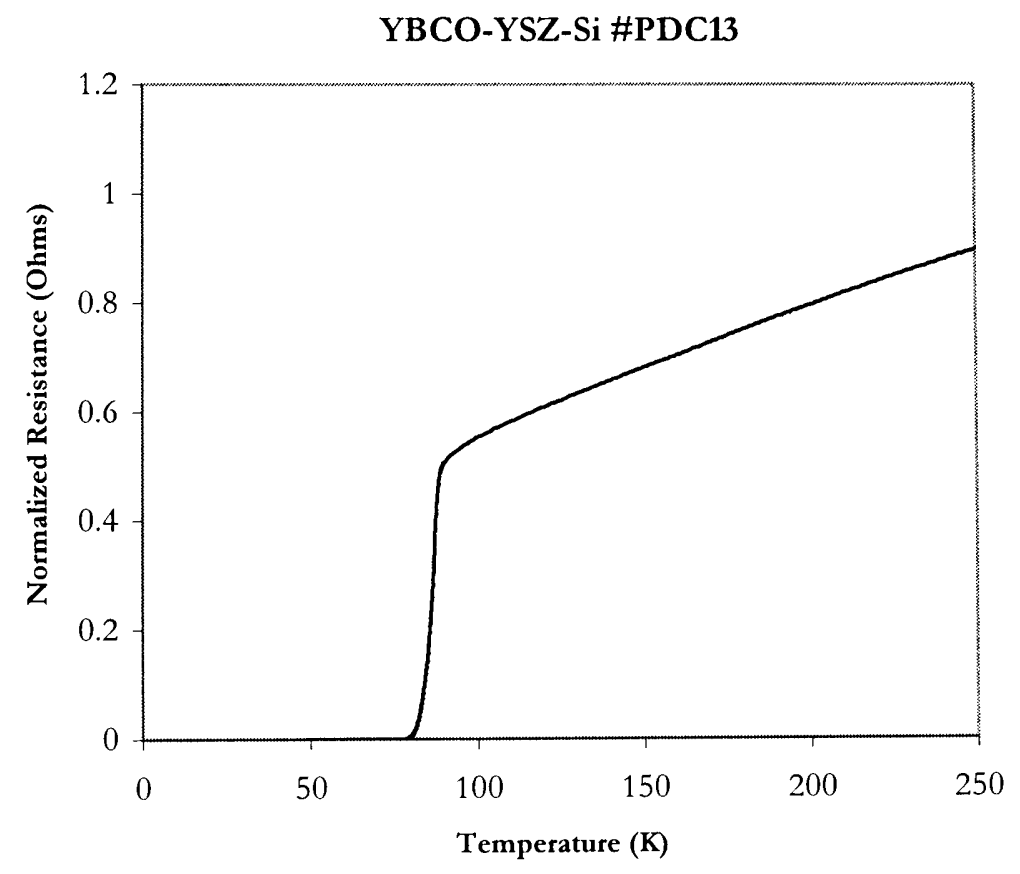

Figure 7.3(b) R vs. T for sample \#PDC13 
The final result is that of sample \#RFN02 and represents the current point in our research. This sample was the first sample that was done using target B, the YSZ target that was fully oxygenated. Being a new target, we again needed to search for new parameters. First we noticed that since the target was fully oxygenated, it was much less conductive than the previous target. As a result pulsed D.C. power was inappropriate for maintaining the plasma discharge (see section 6.1.6). We then had to switch back to R.F. power. However, the advantage of using this target is that no oxygen was required during deposition. The resistance-temperature results of this sample proved to be the best to date. As shown in figure $7.3(\mathrm{c})$, the $T_{c}$ of this sample was at $81 \mathrm{~K}$.

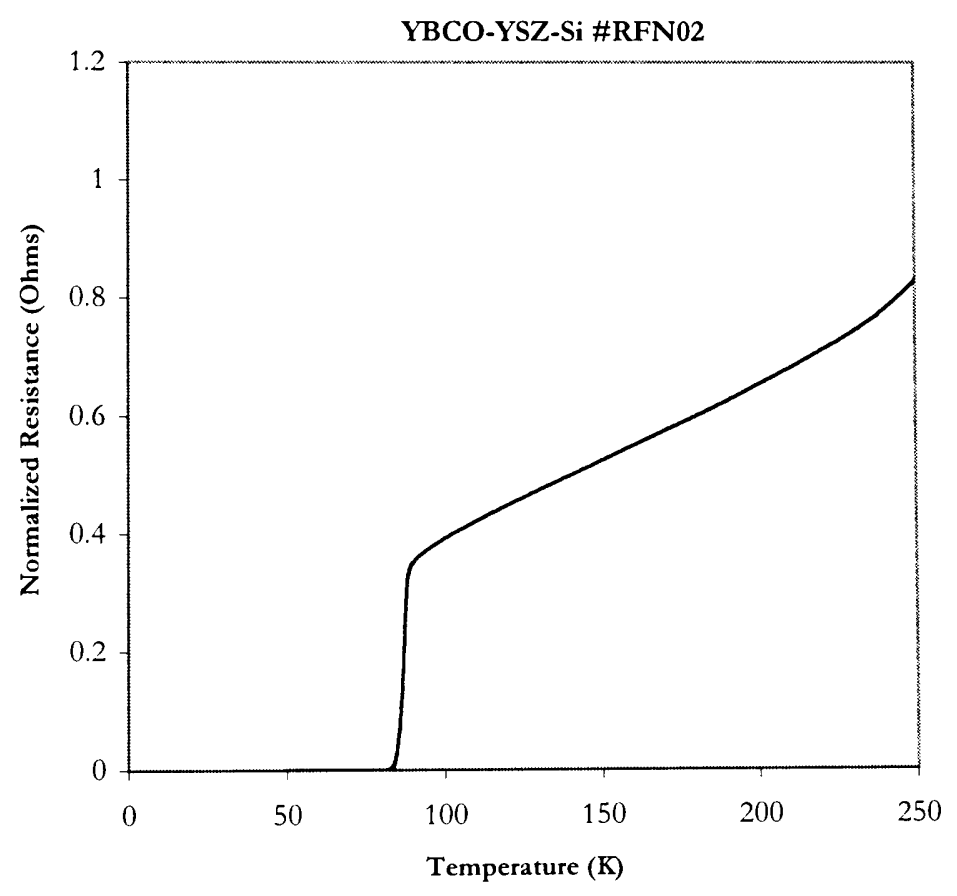

Figure 7.3(c) $\quad \mathrm{R}$ vs. $\mathrm{T}$ for sample \#RFN02 


\section{RESONATOR DESIGN}

\subsection{Introduction}

The best YBCO/YSZ/Si samples obtained from the experiments were used as substrates for fabrication of a co-planar waveguide (CPW) $\mathrm{T}$-resonator. $\mathrm{CPW}$ is favored over microstrip since the superconducting material covers only one side of the substrate. Hence, both ground planes and conductor lie on the same plane. Also, CPW is generally less dispersive than microstrip.

The resonator design uses a short-circuited $\lambda / 2$ meander line attached to a straight thru-line, to realize a resonant circuit with a band reject response. A simplified lumped element model is shown in figure 8.1(a). At odd multiples of $\lambda / 2$, the input signal is shunted to ground, thereby eliminating any response at the resonant frequency. $50 \Omega$ nominal impedance was chosen for the CPW line such that it matched the input impedance of the HP 8753C Network Analyzer used to test the response of the resonator.

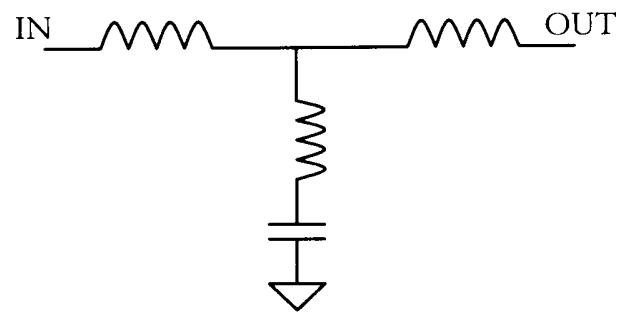

Figure 8.1(a) Lumped element model of $\mathrm{T}$ resonator 
For CPW patterned on a finite-thickness substrate with dielectric constant $\varepsilon_{r}$, the characteristic impedance $Z_{0}$ is given in [112] as:

$$
Z_{01}=\frac{30 \pi}{\sqrt{\varepsilon_{t \pi}}} \frac{K\left(k^{\prime}\right)}{K(k)}
$$

where $K$ is the complete elliptical integral of the first kind.

$\mathcal{E}_{e f f}$ is defined as:

$$
\varepsilon_{t f f}=1+\frac{\varepsilon_{r}-1}{2} \frac{K\left(k^{\prime}\right)}{K(k)} \frac{K\left(k_{1}\right)}{K\left(k_{1}^{\prime}\right)}
$$

with,

$$
\begin{aligned}
& k=\frac{a}{b} ; \quad k_{1}=\frac{\sinh (\pi a / 2 b)}{\sinh (\pi b / 2 b)} \\
& k^{\prime}=\sqrt{1-k^{2}} ; \quad k_{1}^{\prime}=\sqrt{1-k_{1}^{2}}
\end{aligned}
$$

The dimensions of $a, b$ and $b$, are defined as shown in figure 8.1(b).

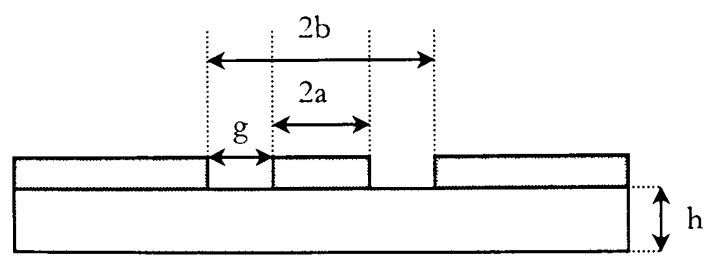

Figure 8.1(b) Co-planar Waveguide Transmission Line 
Here $g$ is the gap spacing and has the value,

$$
g=b-a
$$

The following lists the line parameters used in our design.

\begin{tabular}{|l||l|}
\hline$g$ & $100 \mu \mathrm{m}$ \\
\hline$a$ & $85 \mu \mathrm{m}$ \\
\hline$b$ & $185 \mu \mathrm{m}$ \\
\hline$b$ & $0.5 \mathrm{~mm}$ \\
\hline$\varepsilon_{r}$ & 11.4 \\
\hline
\end{tabular}

CPW line design parameters

With these parameters, the characteristic impedance $Z_{\diamond}$ and the effective dielectric constant $\mathcal{E}_{e f f}$ are calculated to be:

$$
Z_{0}=53.049 \Omega ; \varepsilon_{e f f}=6.096
$$

The resonator was designed for a frequency of $f=1.9 \mathrm{GHz}$. Thus the wavelength $\lambda$ is: 


$$
\lambda=\frac{v_{p}}{f}
$$

where $v_{p}$ is the phase velocity of a wave traveling down the CPW line defined as:

$$
v_{p}=\frac{c}{\sqrt{\varepsilon_{e f f}}}
$$

where $c$ is the speed of light. Hence $v_{p}$ and $\lambda$ are calculated from (44) and (45) to be:

$$
\begin{aligned}
& v_{p}=1.2177 \times 10^{11} \mathrm{~cm} / \mathrm{s}^{2} \\
& \lambda=6.4 \mathrm{~cm}
\end{aligned}
$$

Therefore:

$$
\lambda / 2=3.2 \mathrm{~cm}
$$

This is the length of the meander transmission line section of the resonator. The quality factor, or $\mathrm{Q}$ of the resonator is defined as:

$$
Q=\frac{f_{o}}{B W}
$$

where $f_{o}$ is the center frequency and $B W$ the bandwidth of the resonator. 


\subsection{Design Layout}

Layout of the resonator was done using a standard software-based graphic design tool. The final mask is shown in figure 8.2. This image is a 10:1 scale up of the actual mask. This is done such that when photographed using a standard $35 \mathrm{~mm}$ camera loaded with special lithography film stock, the resulting developed film will have the actual size mask with high line resolution maintained.

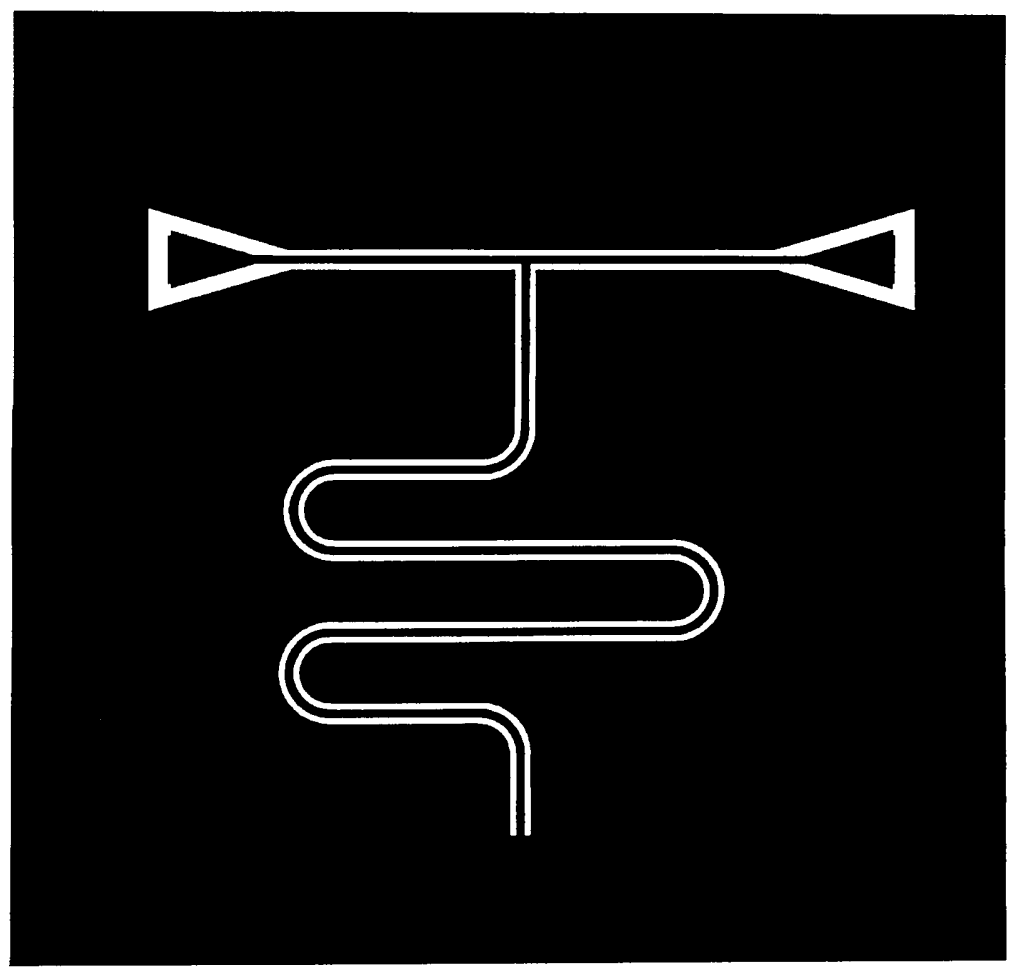

Figure 8.2 Resonator Mask

\subsection{Patterning}

The resonator was patterned onto the YBCO/YSZ/Si substrate using standard UV lithography technique. The sequence of steps is listed below: 
1. Negative photo-resist is spun coated onto the sample at $3000-4000 \mathrm{rpm}$ for 40 seconds.

2. The coated sample is then soft-baked at $80^{\circ} \mathrm{C}$ for 15 minutes.

3. The mask design is placed on top of the sample and clamped down in a clear glass holder and exposed under UV illumination for 3 minutes.

4. The sample is removed from the holder and placed in a standard developer solution for 2-3 minutes.

5. After rinsing with de-ionized water, the sample is blown dry with dry $\mathrm{N}_{2}$ and inspected under a low power microscope to ensure that the design has been properly developed. If not, the process is repeated from step 1.

6. Submerging the sample in 3\% acetic acid for 1-3 minutes carries out etching of the YBCO to obtain the final structure.

\subsection{Testing}

An aluminum test enclosure was fabricated to hold the sample. The enclosure includes two SMA female connectors for interfacing with a network analyzer, and springloaded grounding tabs that attach to the ground planes of the resonator. The resonator was prepared for testing in the following manner.

Tiny indium pellets were first pressed flat at the feed points of the resonator to provide contact with the SMA female connectors' center electrode. The sample was then mounted in the enclosure and spring-loaded grounding tabs were placed over the two 
ground planes to provide a good signal ground path. A glass plate was placed under the sample as a spacer to supplement ground isolation.

The entire package was then placed in a closed-cycle He refrigeration system that was outfitted with feed-through ports for the RF lines, and cooled to $20 \mathrm{~K}$. With an HP 8753C network analyzer connected via the RF lines, measurements of the transmission parameter $\left(\mathrm{s}_{21}\right)$ and the quality factor $(\mathrm{Q})$ were recorded at periodic intervals of temperature.

\subsection{Results}

At room temperature $(290 \mathrm{~K})$ no resonance was observed. Upon cooling to $20 \mathrm{~K}$ a band-reject response was observed at the second harmonic of the design frequency equal to 3.872 $\mathrm{GHz}$ as shown in figure 8.5(a). The results are in agreement with those obtained from simulating the resonator design using the SONNET simulation software tool. The simulated results are shown in figure $8.5(\mathrm{~b})$. 


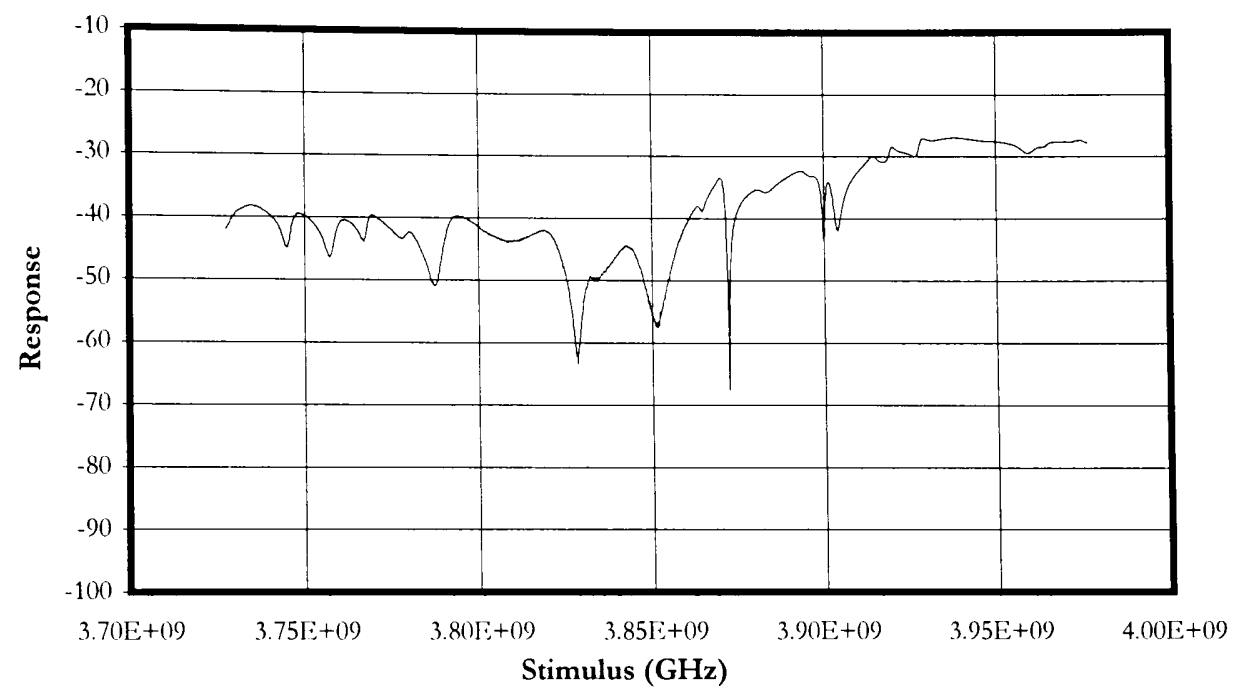

Figure 8.5(a) Experimental Resonator Response

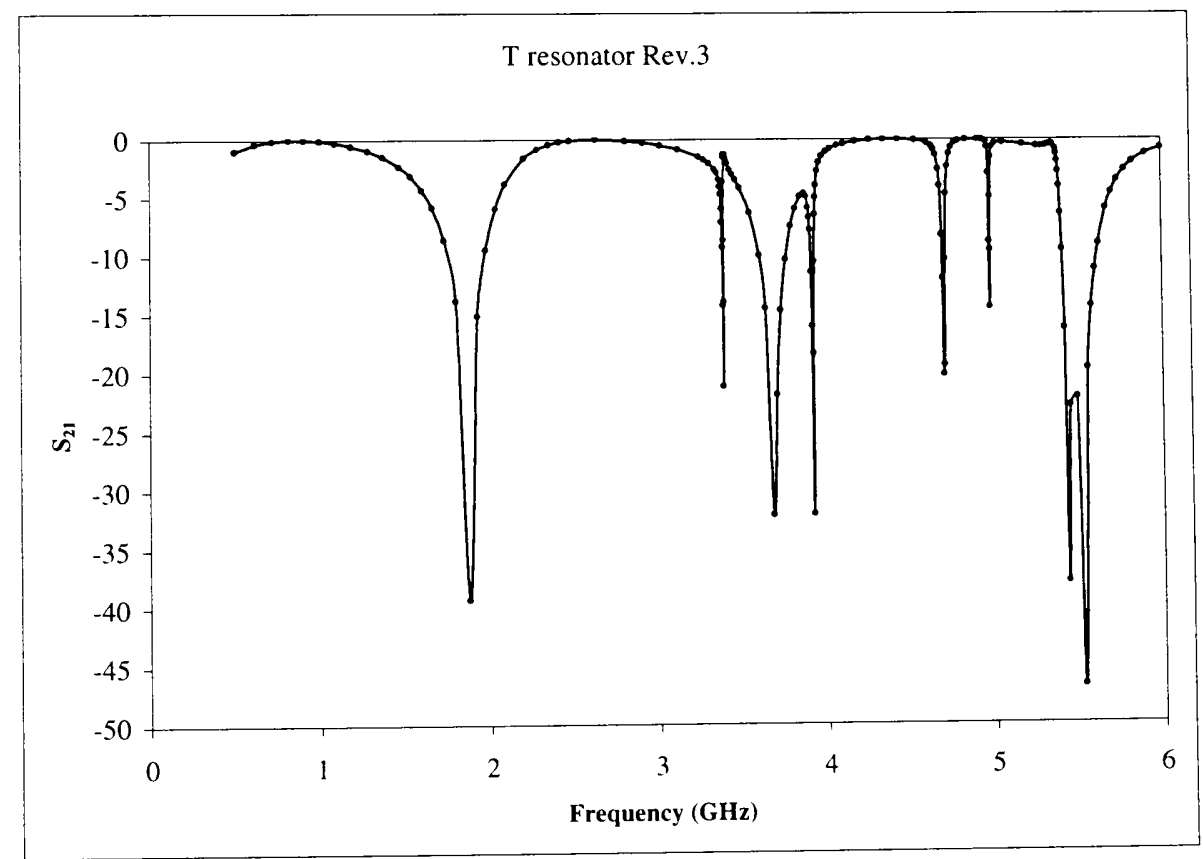

Figure 8.5(b) Simulated Resonator Response 
The change in the response was observed while warming up the sample from $20 \mathrm{~K}$ to room temperature. The values of $s_{21}$ and $\mathrm{Q}$ were recorded every 5 degrees. Figure 8.5(c), shows snapshots of the resonator response as the temperature was increased from $30 \mathrm{~K}-50 \mathrm{~K}$ in $5 \mathrm{~K}$ increments with the $30 \mathrm{~K}$ response denoted by "1" and the $50 \mathrm{~K}$ response denoted by "5". As can be seen, the $\mathrm{Q}$ decreased dramatically over that temperature range to a value of 1500 at $50 \mathrm{~K}$. However, throughout the temperature sweep, the resonant frequency was stationary. This suggests that the resistivity of the silicon is responsible for limiting the $Q$ and that the kinetic inductance of the YBCO film has no effect in shifting the resonant frequency.

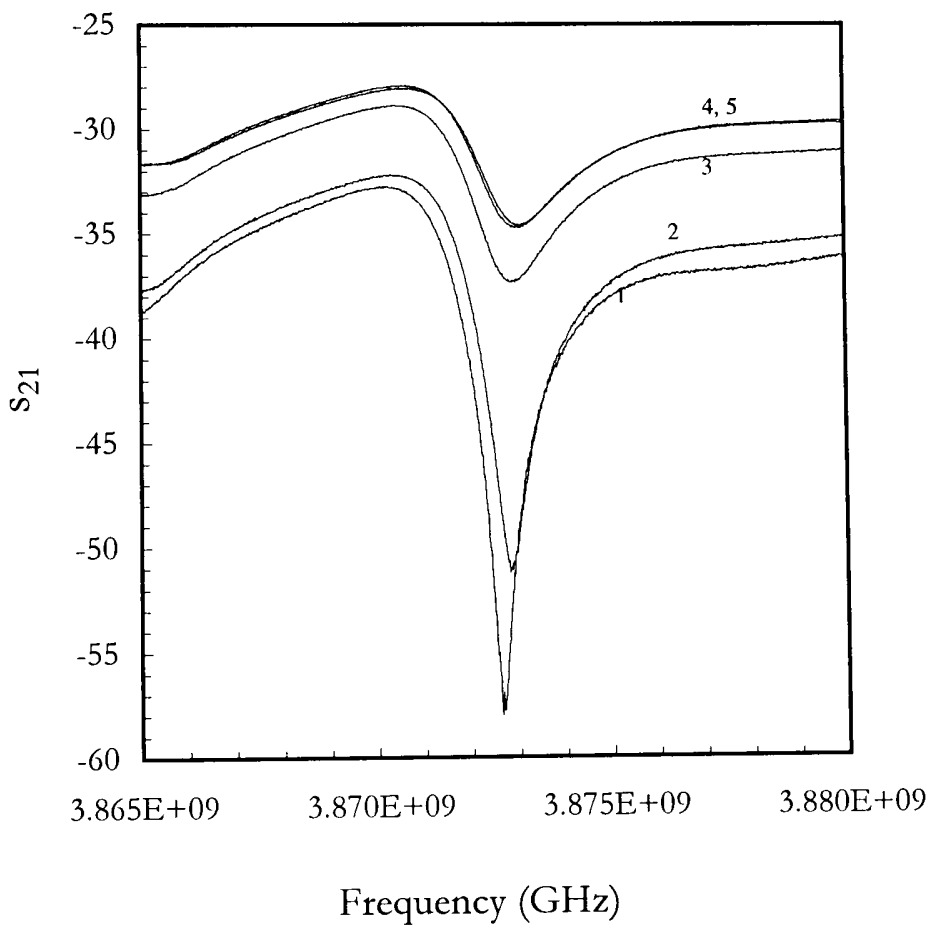

Figure 8.5(c) Resonator Response (30-50K) 
As a comparison, an identical resonator was fabricated using aluminum instead of YBCO. As shown in figure 8.5(d), it is clear that the low surface resistance of the YBCO leads to a resonance with a much higher $\mathrm{Q}$. The $\mathrm{Q}$ of the aluminum resonator was measured at $20 \mathrm{~K}$ to be 6500 .

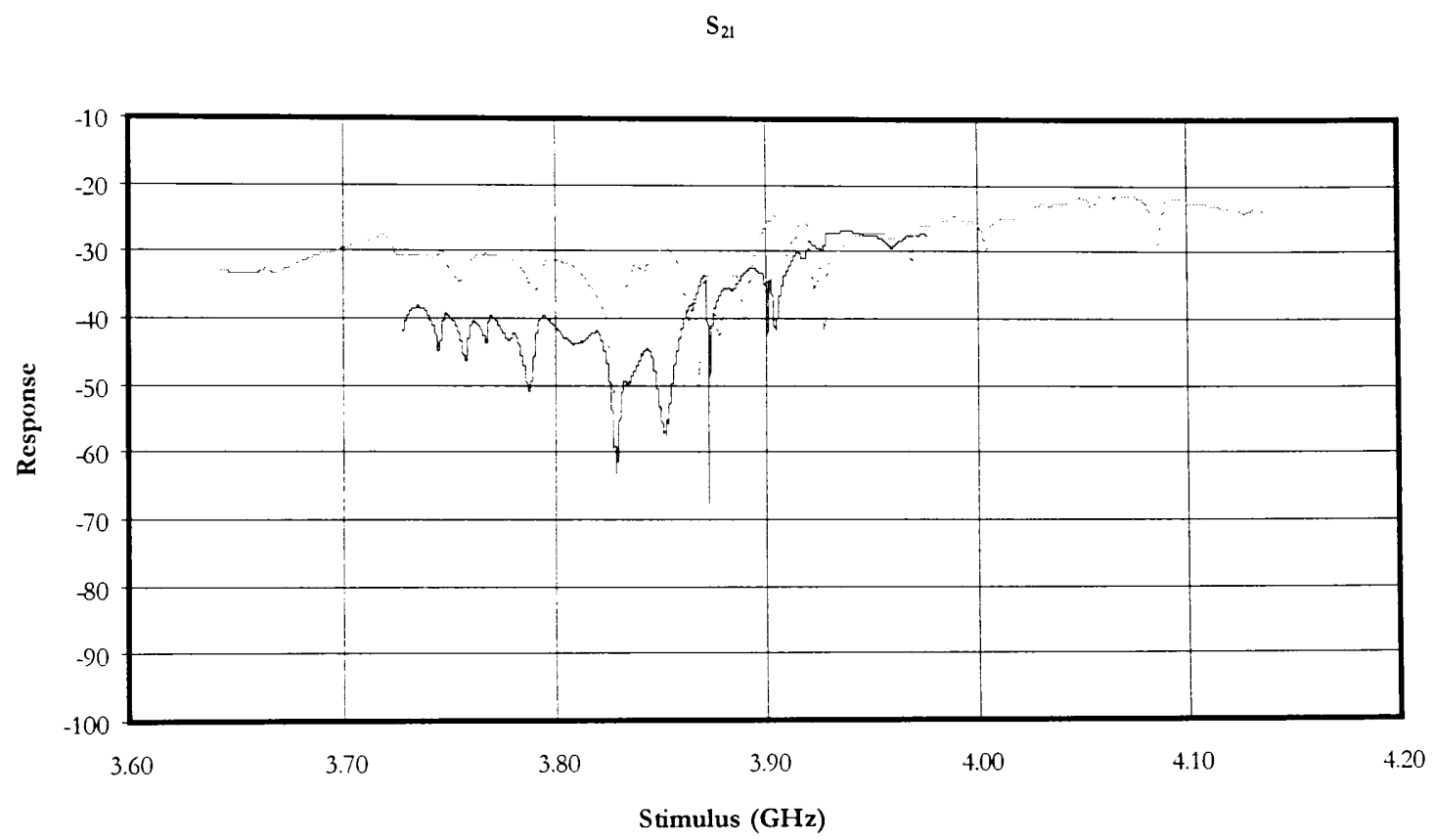

Figure 8.5(d) Comparison of $\mathrm{Al}(--)$ and $\mathrm{YBCO}(--)$ Resonators 
We have seen that silicon buffered with YSZ serves as reasonably good substrate for YBCO thin films. YSZ layers of thickness $50 \mathrm{~nm}$ have shown to be the most successful in providing a stable surface that has a minimum of defects to allow proper growth of YBCO. The role of oxygen is clearly demonstrated in that it influences both the target condition and the substrate. The target is affected by oxygen reducing the ion current, thereby causing less of an ion flux which in turn decreases and/or causes instabilities in the deposition rate. The silicon substrate is readily oxidized at high temperatures in the presence of oxygen and this leads to poor YSZ film growth. By using two targets, one that was fully oxygenated and one that wasn't, we see that in using the fully oxygenated target, no oxygen was required during deposition leading to far superior YSZ films. This result reinforced our conclusion about the role of oxygen in affecting the silicon surface.

With a $\mathrm{T}_{\mathrm{c}(\mathrm{R}=(1)}$ of $81 \mathrm{~K}, \mathrm{YBCO}$ films deposited on the YSZ buffered silicon proved useful enough to pattern a coplanar passive resonator that demonstrated a band-reject characteristic at $3.86 \mathrm{Ghz}$ with a quality factor of $<17,000$.

In the future it is proposed that, for the application of high frequency structures, higher resistivity $(>3000 \Omega-\mathrm{cm}$ ) silicon should be used than the $56 \Omega$-cm currently used. It is thought that this will provide the transmission lines with better isolation from ground, leading to less loss and higher performing superconducting passive elements. 
[1] A. Lauder, K.E. Myers and D. W. Face, Advanced Materials 10, 1249 (1998).

[2] H. Kroger, C. Hilbert, D. A. Gibson, U. Ghoshal and L. N. Smith, Proc. of the IEEE 77, 1287 (1989).

[3] J. Qiao, C. Y. Yang, Mater. Siz. eぇ Eng R14, 157 (1995).

[4] H. K. Onnes, Leiden Comm., 120b (1911).

[5] W'. Meissner and R. Ochsenfeld, Naturwissenchaften 21, 787 (1933).

[6] J. R. Gaveler, Appl. Pbys. Lett. 23, 480 (1973).

[7] J. G. Berdnorz and K. A. Müller, Z. fur Phys. 64, 189 (1986).

[8] M. K. Wu, J. R. Ashburn, C. J. Torng, P. H. Hor, R. L. Meng, L. Gao, Z. J. Huang, Y. Q. Wang and C. W. Chu, Phys. Ret. Lett. 58, 108 (1987).

[9] T. Van Duzer and C. W. Turner, Superconductive Derrices and Circuits, $2^{\text {nd }}$ Ed., New Jersey: Prentice Hall, (1999).

[10] Reported in, Superionductor W'eek 104 (1997)

[11] R. Perin, IEEE Trans. on Magn. 28, 96 (1999).

[12] W. D. Markiewicz, G. M. Clacetta, A. W. Grandin, D. W. Hazelton et al, Adv. in Cro. Eng. 37 Part A, New York: Plenum Press, 361 (1990).

[13] Personal Communication, $6^{\text {th }}$ International Conference on Materials and Mechanisms for High Temperature Superionductors, Houston, TX (2000).

[14] Reported in, Superconductor Week 104 (1997)

[15] Reported in, Superionductor W'eek 113 (1998)

[16] J. Clarke, Nature 333, 29 (1988). 
[18] P. W. Anderson, Pbys. Rev. Lett. 10, 230 (1963).

[19] R. C. Jaklevic, J. Lambe, A.H. Silver, Pbys. Rev. Lett. 12, 159 (1964).

[20] M. Mück, NATO ASI Series on Microwave Superconductivity, Lecture Notes (1999).

[21] C. A. Hamilton, F. L. Lloyd and R. L. Kautz, IEEE Trans. on Magn. MAG-21, 197 (1985).

[22] D. A. Peterson et al, IEEE Trans. on Magn. MAG-21, 200 (1985).

[23] T. H. Geballe, Science 239, 367 (1988).

[24] Reported in, Superconductor Week 12 1, (1999).

[25] J. Rowell, , 6 International Conference on Materials and Mechanisms for High Temperature Superconductors, Houston, TX (2000).

[26] Z. Y. Shen, High Temperature Superconducting Microwave Circuits, Boston: Artech House, 3 (1995).

[27] D. Oates, NATO ASI Series on Microwave Superconductivity, Lecture Notes (1999).

[28] Reported in, Superconductor Week 12 2, (1999).

[29] See [26], 114.

[30] B. Willamsen, NATO ASI Series on Microwave Superconductivity, Lecture Notes (1999).

[31] See [26], 172.

[32] C. Walker, Z. Y. Shen, P. Pang, D. W. Face et al, IEEE Trans. on Microwave Theory and Techniques MTT-39, 1462 (1991).

[33] M. Khun, M. Klinger, A. Baranyak, and J. H. Hinken, IEEE Trans. on Magn. MAG27, 2809 (1991).

[34] R. B. Hammond, G. V. Negrete, M. S. Schmidt, M. J. Moskowitz et al, IEEE MTT-S Int. Microwave Symposium Digest 2, 867 (1990).

[35] H. S. Newman, D. B. Chrisey, J. S. Horwitz, B. D. Weave, and M. E. Reeves, IEEE Trans. on Magn. MAG-27, 2540 (1991).

[36] D. B. Rensch, J. Y. Josefowicz, P. MacDonald, C. W. Niel, et al, IEEE Trans. on Magn. MAG-27, 2553 (1991). 
[37] J. H. Takemoto, C. M. Jackson, R. Hu, J. F. Burch, K. P. Daly, and R. W. Simon, IEEE trans. on Magn. MAG-27, 2549 (1991).

[38] J. Bardeen, L. N. Cooper, J. R. Schrieffer, Pbys. Rev. 108, 1175 (1957).

[39] M. Tinkham, Introduction to Superionductinity, $2^{\text {nd }}$ Ed., New Jersey: Prentice Hall, 43 (1999).

[40] S. - A. Zhou, Electrodynamics of Solids and Microwave Superconductivity, New York: John Wiley \& Sons, 333 (1999).

[41] V. L. Ginzburg, L. D. Landau, Zh. Eksp. Teor. Fi . 20, 1064 (1950).

[42] D. Saint-James, E. J. Thomas and G. Sarma, Type II Superconductivity, Oxford: Pergamon (1969).

[43] F. London and H. London, Proceedings of the Royal Society A149, 71 (1935).

[44] See [39], 18.

[45] D. Shoenberg, Superionductivity, Cambridge: Cambridge University Press, 194 (1965).

[46] See [9], 124.

[47] T. Trachoma, K. Minima, K. Yamamoto, K. Hirata, et al, Japan. J. of Appl. Pbys. 28, L987 (1989).

[48] R.W. Simon, C.E. Platt, A. E. Lee, C. S. Lee, et al, Appl. Pbys. Let. 53, 2677 (1988).

[49] R. L. Sandston, E. A. Gneiss, W. J. Gallagher, A. Sampler et al, Appl. Phys. Lett. 53, 1874 (1988).

[50] T. Konaka, M. Sato, H. Asano, and S. Kubo, Journal of Superconductivity 4, 283 (1991).

[51] See [50].

[52] M. G. Norton, L. A. Teitz, S. A. Summerfelt, and C. B. Carter, Appl. Phys. Lett. 55, 2348 (1989).

[53] G. Koren, E. Polturak, B. Fisher, D. Cohen, and G. Kimel, Appl. Phys. Lett. 53, 2330 (1988).

[54] M. Hao, K. Lai, W. M. Chen and J. C. Lee, Appl. Phys. Lett. 65, 1133 (1994).

[55] See [40], 442 . 
[56] S. K. Tewsbury and L. A. Hornak, IEEE Circuits and Devices Mag. 5, 22 (1989).

[57] A. Mogro-Campero, Supercond. Sci. Technol 3, 155 (1990).

[58] H. Myoren, Y. Nishiyama, H. Nau, T. Imura, Y. Osaka, S. Yamanaka and M. Hattori, Jpn. J. Appl. Phys, 27, L1068 (1988).

[59] K. Harada, N. Fujimori and S. Yazu, Jpn. J. Appl. Phys. 27, L1524 (1988).

[60] H. Mori and H. Ishiwara, Jpn. J. Appl. Phys. 30, 1145 (1991).

[61] T. Inoue, Y. Yamamoto, S. Koyama, S. Suzuki and Y. Ueda, Appl. Phys. Lett. 56, $1332(1990)$.

[62] H. Fukumoto, T. Imura and Y. Osaka, Appl. Phys. Lett. 54, 360 (1989).

[63] X. D. Wu et al, Appl. Phys. Lett. 54, 243 (1989).

[64] S. Miura, T. Yoshitake, S. Mastubara, Y. Miyasaka, N. Shohata and T. Satoh, Appl. Phys. Lett. 53, 1967 (1988).

[65] R. Soto, Sputtering of Magnesium Oxide, Master's Thesis: Florida International University (1997).

[66] T. Khan, Sputtering of Zirconium Oxide for High Temperature Superconducting Thin Films, Master's Thesis: Florida International University (1999).

[67] A. Lubig, Ch. Buchal, J. Shubert, C. Copetti, and D. Guggi, C. L. Jia and B. Stitzker, J. Appl. Phys. 71, 5560 (1992).

[68] A. Bardal, M. Zwerger, O. Eibl, J. Wecker and Th. Mathée, Appl. Phys. Lett. 61, 1243 (1992).

[69] Th. Mathée, J. Wecker, H. Behner, G. Friedl, O. Eibl, and K. Samwer, Appl. Phys. Lett. 61, 1240 (1992).

[70] Yu. A. Boikov, Z. G. Ivanov, T. B. Zhukova, G. Brorsson, E. Olsson and T. Claeson, Sov. Tech. Phys. Lett. 18, 622 (1992).

[71] D. B. Fenner, A. M. Viano, D. K. Fork, G. A. N. Connell, J. B. Boyce, F. A. Ponce and J. C. Tramontana, J. Appl. Phys. 69, 2176 (1991).

[72] D. K. Fork, D. B. Fenner, G. A. N. Connell, J. M. Phillips and T. H. Geballe, Appl. Pbys. Lett. 57, 1161 (1990). 
[73] S. Horita, T. Tajima, M. Murakawa, T. Fujiyama, and T. Hata, Thin Solid Films 229, 17 (1993).

[74] M. Nagashima, S. Nakano, K. Sasaki, and T. Hata, Jpn. J. of Appl. Pbys. 38 Pt. 2 1A/B, L74 (1998).

[75] M. Spankova, S. Gazi, S. Chromik, A. Rosova, I. Vavra, and S. Benaka, J. of Low Tem. Pbys. 106 3/4, 439 (1997).

[76] W. - C. Tsai and T. - Y. Tseng, Thin Solid Films 306, 86 (1997).

[77] C. - Y. Hung, J. M. Van Scyoc, T. E. Schlesinger, J. C. Johnson et al, Mater. Sci. do Eng. B33, 85 (1995).

[78] F. Schmidt, D. Boshetto, S. Linzen, J. Kräußlich et al, Pbysica C 326-327, 99 (1999).

[79] F. Benyaich, F. Prolo, E. Rimini, C. Spinella and P. Ward, J. Appl. Pbys. 71, 638 (1992).

[80] S. Ben Amor, B. Rogier, G. Baud et al, Mater. Sic. é Eng. B57, 28 (1998).

[81] A. Belkind, A. Freilich, and R. Scholl, J. L'ai. Sii. Technol. A17, 1934 (1999).

[82] W. M. Posadowski, Thin Solid Films 343, 85 (1999).

[83] A. Billard and C. Frantz, J. de Phys. III (France) 6, 1181 (1996).

[84] D. K. Fork, S. M. Garrison, M. Hawley and T. H. Geballe, J. Mater. Res. 7, 1641 (1992).

[85] A. L. Vasiliev, E. Olsson, Ju. Boikov, T. Claeson, N. A. Kiselev, Physica C 253, 297 (1995).

[86] Michael A. Lieberman and Allan J. Lichtenberg, Principles of Plasma Discharges and Materials Processing, New York: John Wiley \& Sons Inc (1994).

[87] D. L. Smith, Thin Film Deposition, New York: McGraw-Hill (1968).

[88] L. A. Azimovich, Elementary Plasma Physics, Blaisdell Publishing, Waltham (1965).

[89] J. L. Vossen and J. J. Cuomo, "Glow Discharge Sputter Deposition", Thin Film Processes, eds. J.L. Vossen and W. Kern, New York: Academic Press (1978).

[90] R. A. Powell, S. Rossnagel, PI D for Microelectronics: Sputter Deposition applied to Semiconductor Manufacturing Thin Films 26, Boston: Academic Press (1999). 
[91] M. Ohring, The Materials Science of Thin Films, California: Academic Press (1990).

[92] R. Parsons, "Sputter Deposition Processes", Thin Film Processes II, ed. John L. Vossen, Boston: Academic Press (1991).

[93] H. R. Koenig and L. I. Maissel, IBM J. Res. Dev. 14, 168 (1970).

[94] S. Schiller et al, Surf. Coat. Tech. 61, 331 (1993).

[95] O. Zywitzki et al, Surf. Coat. Tech. 82, 169 (1996).

[96] W. D. Sproul et al, J. Vac. Sii. Technol. A13, 1188 (1995).

[97] J. M. Schneider and W. D. Sproul, 98/1 Reactive Sputtering, ed. W. D. Westwood, Philadelphia: Institute of Physics, A5.1:1 (1998).

[98] RPG 50 User's Manual, ENI Corp., (1995).

[99] J. S. Chapin, IBM J. Res. Dev. 25, 37 (1974).

[100] R. K. Waits, "Planer Magnetron Sputtering”, Thin Film Processes, ed. By John L Vossen, Boston: Academic Press, (1978).

[101] J. A. Thornton and A. S. Penfold, Thin Film Processes, ed. By John L Vossen, Boston: Academic Press, (1978).

[102] S. Schiller, V. Heisig, and K. Goedicke, Thin Solid Films 40, 217 (1977).

[103] J. A. Thornton, J. Vac. Si. Technol. 15, 171 (1978).

[104] S. M. Rossnagel, "Glow Discharge Plasmas and Sources for Etching and Deposition", Thin film Processes II, ed. By John L Vossen, Boston: Academic Press, (1991).

[105] J. A. Thornton, “Coating Deposition by Sputtering”, Deposition Technologies for Films and Coatings, ed. R. F. Bunshah, New Jersey: Noyes Publications.

[106] B. Window, J. of Vac. Sir. and Technol. A4, 196 (1986).

[107] N. Savidis, J. of Vac. Sci. and Technol. A4, 453 (1986).

[108] C. S. Liang, Applied Electromagnetism, Boston: PWS Publishing, (1995).

[109] P. D. Townsend, Ion Implantation, Sputtering and Their Applications, Boston: Academic Press, (1976). 
[110] M. Hein, NATO ASI Series on Microwave Superconductivity, Lecture Notes (1999).

[111] J. M. Phillips, High Temperature Superconducting Materials Sizence and Engineering: New Concepts and Technology, ed. Donglu Shi, North Holland: Pergamon (1995).

[112] R. Mongia, I. Bahl and P. Bharita, RF and Microwave Coupled Line Circuits, Boston: Artech House (1999).

[113] B. Chapman, Glow Discharge Processes, New York: John Wiley \& Sons (1980). 